\title{
Active Sites of the Multi-subunit RNA Polymerases of Eubacteria and Chloroplasts are Similar in Structure and Function
}

\author{
Peramachi Palanivelu* \\ Department of Molecular Microbiology, School of Biotechnology, Madurai Kamaraj University, \\ Madurai - 625 021, Tamil Nadu, India; ppmkupp@gmail.com
}

\begin{abstract}
Objectives: To analyze and compare the Multi-subunit (MSU) DNA dependent RNA Polymerases (RNAPs) of eubacteria and chloroplasts and to identify the extent of conservations between them with special reference to the active sites. Methods: The advanced version of Clustal Omega was used for protein sequence analysis of the MSU DNA dependent RNAPs from eubacteria and chloroplasts. The conserved motifs identified by the bioinformatics analysis and the biochemical and Sitedirected Mutagenesis (SDM) experiments and X-ray crystallographic analysis data available on the eubacterial MSU RNAPs are used to derive and analyze active site regions of the MSU RNAPs of chloroplasts. Findings: Multiple Sequence Alignment (MSA) of RNAPs from both the sources showed many highly conserved motifs among them. The possible catalytic regions in the catalytic subunits $\beta$ and $\beta^{\prime}$ of eubacteria and their counterparts, viz. $\beta, \beta^{\prime}$ and in chloroplasts RNAPs consist of an absolutely conserved catalytic amino acid R, in contrast to a K as reported for DNA polymerases and Single Subunit (SSU) RNAPs. Besides, the invariant 'gatekeeper/DNA template binding' YG pair is also found to be absolutely conserved in the MSU RNAPs of chloroplasts, as reported in SSU, MSU RNAPs and DNA polymerases. The eubacterial $\beta$, the initiation subunit, is highly homologous to $\beta$ subunit of chloroplast MSU RNAPs, i.e., the eubacterial and chloroplast $\beta$ subunits exhibit very similar active site motifs, catalytic regions and distance conservations between the template binding YG pair and the catalytic R. However, the bacterial $\beta^{\prime}$ elongation subunit is not completely similar to the $\beta^{\prime}$ elongation subunit of chloroplasts, but partly similar to the $\beta^{\prime}$ and $\beta^{\prime \prime}$ subunits of chloroplast RNAPs. Interestingly, MSA analysis shows that the active sites are shared between $\beta^{\prime}$ and $\beta^{\prime \prime}$ in the MSU RNAPs of chloroplasts, i.e., the metal binding site is found in the $\beta^{\prime}$ subunit whereas the catalytic regions are located in $\beta^{\prime \prime}$ subunit of chloroplast MSU RNAPs. Another interesting finding is, in the elongation subunits, i.e., in the eubacterial $\beta^{\prime}$ and the chloroplast $\beta^{\prime \prime}$ catalytic subunits, the proposed catalytic R is placed at double the distance, i.e., -16 amino acids downstream from the YG pair, in contrast to SSU RNAPs and DNA polymerases where the distance is only 8 amino acids downstream from the YG pair. An invariant $\mathrm{Zn}^{2+}$ binding motif reported in the eubacterial elongation subunit, viz., $\beta^{\prime}$ is found in the $\beta^{\prime \prime}$ subunits of chloroplasts. Applications: Analysis of MSU RNAPs of chloroplasts assumes greater importance as it is the one that transcribes the foreign genes in chloroplast transformation experiments.
\end{abstract}

Keywords: Chloroplast Multi-subunit RNA Polymerases, Distance Conservations, Eubacterial RNA Polymerases, Metal Binding Regions, Multi-subunit RNA Polymerases, Plastid Encoded Polymerases, Polymerase Active Sites

\section{Introduction}

RNAPs (EC 2.7.7.6) are key enzymes, found in all living cells that perform the flow of genetic information from
DNA $\rightarrow$ RNA. Thus, they play a vital role in the control of gene expression at the transcription level. Interestingly, the sequences, common structural framework and functions of these MSU DNA dependent RNA

${ }^{*}$ Author for correspondence 
polymerases are universally conserved in bacteria, fungi, plants and animals ${ }^{1}$. Understanding the overall structure, mechanism and regulation of the RNAPs are the primary goal of molecular biologists since its discovery. Though RNAPs are found in all species across all three kingdoms, their number and composition vary across taxa. For example, viruses contain mainly two types of RNAPs, viz. DNA dependent RNAPs and RNA dependent RNAPs. Both eubacteria and archaebacteria contain a single type of RNAP of Multi-subunit type. In eubacteria, the MSU RNAP core enzyme is composed of 5 subunits, while eukaryotes contain at least five distinct types of RNAPs (I-V), which are also MSU enzymes but made up of 12-14 subunits. In spite of such differences, there are striking similarities among the transcriptional mechanisms by various types of RNAPs $\stackrel{1}{-}$. Chloroplasts in higher plants use two different types of RNAPs, one is the nuclear-encoded SSU type and the other one is the chloroplast-encoded (PEP) MSU type ${ }^{2,3}$. The MSU RNAPs of eubacteria and PEP are basically similar in structure and functions ${ }^{3}$. Large volumes of protein sequence data are available for most of the prokaryotic and chloroplast MSU RNAPs. Understanding the biological principles buried in these sequencing data is a significant challenge for scientists. A great deal of information is available on the MSU RNAPs of eubacteria. However, data on the conserved motifs and active site analyses of the chloroplast MSU RNAPs are very limited. Analyses of chloroplast MSU RNAPs assume greater importance as it is the one that transcribes the foreign genes in chloroplast transformation experiments ${ }^{4}$. Therefore, a comparative analysis of the protein sequences of both the MSU enzymes was performed to find out the conserved motifs and active sites in the chloroplast MSU RNAPs, using the data already available on the eubacterial enzymes.

RNAPs catalyze the chemical reactions that synthesize an RNA strand, using the DNA template with all the 4 NTPs and a metal ion. The RNA synthesis involves three steps, viz. initiation, elongation and termination. The newly formed RNA copies serve as blueprints for protein synthesis during the next step of translation. The basic transcription unit is the distance between the sites of Transcription Start Site (TSS) and Transcription Termination Site (TTS) and may have one or more genes (e.g., mono or poly-cistronic mRNAs). For the discussion on the discovery of RNAP, dynamics of transcription refer to Palanivelu ${ }^{5}$.

\section{Types of DNA Dependent MSU RNAPs}

There are at least 4 different types of MSU RNAPs in living cells, viz.

- DNA dependent MSU RNAP of eubacteria.

- DNA dependent MSU RNAP of archaebacteria.

- DNA dependent MSU RNAP of chloroplasts (Plastid encoded).

- DNA dependent MSU RNAPs of eukaryotes.

In my earlier communications, SSU RNAPs (viral types) and MSU RNAPs from eubacteria were analyzed in detail and reported ${ }^{5,6}$. In this communication, MSU RNAPs of chloroplasts (prokaryotic type, plastidencoded) are analyzed for their conserved motifs, active sites, metal binding regions. Based on MSA data and with the biochemical, SDM and X-ray crystallographic data available from their counterpart MSU enzyme from eubacteria, a consensus model for the initiation and elongation cycles are proposed.

\section{MSU DNA dependent RNAPs of Chloroplasts}

\subsection{Structural Features of the PEP and its similarity with Eubacterial Enzyme}

As the chloroplasts are known to have inherited the PEP from cyanobacteria by endosymbiosis about $1 \times 10^{9}$ years ago, it is also composed of the same core subunits, viz. $\alpha, \beta$, $\beta^{\prime}$ and $\beta^{\prime \prime}$. However, the rpoC gene coding for the $\beta^{\prime}$ subunit in eubacteria is split into $\operatorname{rpoC} 1\left(\beta^{\prime}\right)$ and $\operatorname{rpoC} 2\left(\beta^{\prime \prime}\right)$ in chloroplasts. Thus, the MSU enzyme from chloroplasts is very similar to the eubacterial counterparts, except having an additional subunit, i.e., $\beta$ " in its structure and hence the chloroplasts' core enzyme is composed of $\alpha, \beta, \beta^{\prime}, \beta^{\prime \prime}$ and $\omega$-subunits (Figure 1) and encoded by rpoA, rpoB, rpoC1, rpoC2 and rpoZ genes, respectively. When a $\sigma$ subunit (nuclear-encoded) is associated with the core polymerase, the PEP holoenzyme is formed, which can initiate transcription at specific sites on the chloroplast DNA templates. Figure 1 shows a schematic diagram of a chloroplast MSU RNAP. MSA shows that the plastid-encoded MSU RNAP subunits $\alpha, \beta, \beta^{\prime \prime}$ are homologous and functionally similar to the $\alpha$, $\beta$ and $\beta^{\prime}$ subunits of eubacterial RNAPs, respectively. 


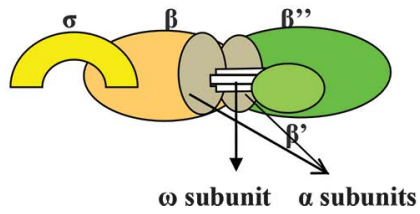

Figure 1. A schematic diagram of a chloroplast MSU RNAP with its subunits.

(The elongation subunits, viz. $\beta^{\prime}$ harbours the metal binding site whereas, the $\beta^{\circ}$ possesses the catalytic site)

\subsection{PEP and NEP are Structurally and Functionally Different}

Chloroplasts contain a circular, double-stranded genome of sizes ranging from 100 to $200 \mathrm{~kb}$ and harbour $\sim 120$ genes. 4 . For example, the Zea mays chloroplast genome is of $140 \mathrm{~kb}$ in size and contains 70 protein-coding genes, 30 tRNAs and 4 rRNAs). Chloroplasts in higher plants use two different types of RNAPs to transcribe all its genes. One is the nuclear-encoded SSU RNAP (NEP), which is homologous and very similar to SSU viral and mitochondrial polymerases 5 and the second one is the plastid-encoded MSU RNAP (PEP), which is structurally and functionally very similar to the eubacterial MSU RNAPs (this communication). The SSU RNAP, encoded by the nucleus is structurally unrelated to PEP and belongs to the "SSU RNAP" protein family which are similar to the RNAPs of bacteriophages T3, T7, SP6, etc. in structure and function ${ }^{-\underline{7}}$. The NEP mainly involves in the transcription of non-photosynthetic housekeeping genes ${ }^{8,9}$ whereas the PEP mainly involves in transcription of the photosynthesis-related genes and tRNAs ${ }^{10}-\underline{12}$. This is evident from the knockout mutants of PEP that show an albino phenotype and lack photosynthesis ${ }^{13}$. Thus, both PEP and NEP are essential for transcription of all chloroplast genes but each transcribes a distinct group of genes. Thus, the PEP and NEP operate independently. For example, Serino and Maliga have reported that deletion of each of the PEP genes yielded photosynthetically defective plants that lack PEP activity while maintaining transcription specificity from NEP promoters ${ }^{14}$. It is interesting to note that the rpoB operon encoding 3 of the 4 PEP core subunits in all higher plants is solely transcribed by NEP${ }^{2}$. Genes encoding the core subunits of PEP (rpoA, B, C1 and C2) are located in the chloroplast genome itself, but those encoding the $\sigma$ factors, which are required for promoter recognition and transcription initiation are located in the nuclear genome ${ }^{15}$.

NEP and PEP use different promoters. However, many plastid genes have both PEP and NEP promoters. Most NEP promoters have the conserved YRTA motif and most of the PEP promoters resemble bacterial ' $\sigma 70$ promoters type' and typically characterized by -10 and -35 consensus sequence motifs. However, a number of PEP promoters lack the -10 or the -35 elements, a few even both $\frac{16,17}{17}$. Although plastids possess genes for the core subunits of a PEP, this enzyme can only correctly initiate transcription together with nuclear-encoded $\sigma$ factor(s). Therefore, transcription by PEP in chloroplasts is controlled by the nucleus by providing the $\sigma$ factor and the NEP. Furthermore, the transcriptional activity of NEP and PEP is also affected by endogenous and exogenous factors.

\section{Transcription Process in Chloroplasts and Eubacteria}

The genetic material of chloroplasts is organized in the same way as bacterial nucleoids. The core structures of the chloroplast enzyme and its regulatory DNA sequences for transcription exhibit a strong homology with their E. coli counterparts as discussed elsewhere. As the subunit structures of both the MSU RNAPs are very similar with large numbers of highly conserved motifs, the initiation, elongation and termination events should be also very similar to eubacteria as reported ${ }^{6}$. This is further elaborated in this communication.

\section{Materials and Methods}

A large number of MSU RNAPs from eubacteria and chloroplasts have been isolated, purified, characterized, cloned and sequenced ${ }^{1}-\underline{3}, \underline{18}$ and references therein. Complete nucleic acid and protein sequence data are available for many of these enzymes from different sources. Thus, these data have become valuable tools in analyzing and understanding the structure-function relationships of the chloroplast enzymes. For MSA of various MSU RNAPs, the sequences were retrieved from SWISS-PROT and PUBMED sites and analyzed using Clustal Omega, an accurate, fast and widely accepted algorithm, available on their website. This communication also presents a 
consensus model for initiation and elongation processes for PEPs similar to the eubacterial MSU RNAPs.

\section{Results and Discussion}

\subsection{MSA DNA dependent MSU RNAPs of Chloroplasts from Different Sources}

Figures 2-7 shows the MSA of various subunits of MSU RNAPs from chloroplasts and the mix and match analysis with their counterparts of the eubacterial MSU RNAPs. Only the relevant and highly conserved regions are shown (Figures 2-7). The catalytic, template and substrate binding regions are highlighted in yellow. The possible and experimentally proved metal binding regions (eubacteria) and proposed regions are highlighted green. The protein sequences of standard organisms are highlighted in magenta and used for numbering.

\subsection{MSA of a Subunits of MSU RNAP from Chloroplasts}

MSA analysis of the a subunits of chloroplast MSU RNAPshas shown that they are highly conserved and showed long stretches of conserved motifs among them. Unlike the a subunits of eubacteria, the a subunits chloroplasts MSU RNAPs showed two invariant YG pairs and an LG pair suggesting their possible role in template binding (data not shown).

\subsection{MSA of $\beta$ Subunits MSU RNAP from Chloroplasts}

Figure 2 shows the MSA and conserved motifs in the $\beta$ subunits of MSU RNAPs from chloroplasts. There are large numbers of conserved motifs among them and some are found to be very long stretches (highlighted). The Zea mays $\beta$ subunit is used as the standard for numbering and highlighted in magenta. As seen in the eubacterial $\beta$ subunits, the 'gatekeeper pair' YG and the catalytic $\mathrm{R}$ are strictly conserved, including the distance conservation here also (highlighted). Furthermore, the catalytic R and the YG pair maintain a distance of 7 amino acids as seen in eubacterial $\beta$ subunits. This strongly suggests that the DNA polymerases, SSU and MSU RNAPs use a similar set of amino acids for the template, substrate binding and catalysis, establishing a structure-function relationship among the nucleotidyl transferases. The immediate downstream amino acid from the catalytic $\mathrm{K}$ in DNA polymerases is usually a $\mathrm{G}$ or $\mathrm{A}^{19}$ but in viral RNAPs, it is an $\mathrm{R}$ or sometimes a $\mathrm{K}$, in eubacterial MSU RNAP $\beta$ subunits, it is an invariant $D$ and here it is $G / D$. An invariant $R$ is found $-7 / 8$ amino acids downstream from the catalytic $\mathrm{R}$ (Table 1). Unlike the $\beta$ subunit of eubacterial RNAPs, which possess three YG pairs, the chloroplast $\beta$ subunit showed an invariant YG pair, an LG pair and two FG pairs suggesting at least three of them may possibly involve in the 'three-point attachment' on the template. Other than some of the viral SSU RNAPs, most of the SSU RNAPs use only one template binding YG pair ${ }^{5}$. The DNA polymerases also use only one YG pair for template binding ${ }^{19}$. In only about ten organisms C-terminal region is found to be highly conserved as indicated in Figure 2.

\subsection{MSA of $\beta$ ' Subunits of the MSU RNAPs from Chloroplasts}

Figure 3 shows the MSA and conserved motifs in the $\beta$ ' subunits of MSU RNAPs of chloroplasts. There are about a dozen conserved motifs among them (highlighted) with one or two long stretches around the metal binding site. The Zea mays $\beta$ ' subunit is used as the standard for numbering and highlighted in magenta. Possible metal binding sites are highlighted in green. As seen in eubacterial $\beta, \beta$ ' and chloroplast $\beta$ subunits, no 'gatekeeper YG pair' and the corresponding catalytic $\mathrm{R}$ are found in the $\beta$ ' subunits of chloroplasts RNAPs, suggesting that this subunit may not directly involve in catalysis as the catalytic $\mathrm{R}$ and the YG pair is found to be present in the $\beta$ " subunit only. However, the absolutely conserved metal binding motif -NADFDGDQMA - is found in this $\beta$ 'subunit as seen in $\beta^{\prime}$ subunits of eubacteria ${ }^{20}$, suggesting that the active site regions are split or shared between the $\beta$ ' and $\beta$ " subunits in chloroplast MSU RNAPs. No conserved motif is found in the C-terminal region as seen in the $\beta$ and $\beta$ ' subunits of eubacterial MSU RNAPs. In the absence of an YG pair, an FG and two LG pairs are seen (highlighted) in these subunits suggesting a possible interaction with the template by this subunit also. However, the absence of an invariant $Y G$ pair and its catalytic $R$ at downstream from the YG pair at the expected distance suggests that this subunit may be playing only a supporting metal binding role in the transcription process. This can be proved 


\section{CLUSTAL O (1.2.4) MSA of the $\boldsymbol{\beta}$ subunits of various chloroplast of MSU RNAPs}

YP 009104799.1 YP_009106550.1 YP- 009106801.1 YP 009093042.1 NP_043712.1 YP 009093282.1 ADB27491.1 YP 004072537.1 YP_009093440.1 $\mathrm{AE} \overline{\mathrm{X}} 37816.1$ YP 003735004.1 ADĪ 40219.1 ADI 40351.1 NP 039275.1 NP-043015.1 CĀ̄ 60276.1 ARK14872.1 YP 009367394.1 AAV̄ 80598.1 ABB81942.1 YP 009106261.1 YP 009106417.1 AIT̄95281.1 YP_009105929.1

YP_009104799.1 YP 009106550.1 YP_009106801.1 YP_009093042.1 NP 043712.1

YP-009093282.1 ADE 27491.1 YP_004072537.1 YP 009093440.1 AEX̄ 37816.1 YP_003735004.1 ADI 40219.1 ADI 40351.1 NP 039275.1 NP- 043015.1 CAA60276.1 ARK1 4872.1 YP_009367394.1 AAV̄80598.1 ABB81942.1 YP_009106261.1 YP 009106417.1 AIT95281.1 YP 009105929.1

YP 009104799.1 YP 009106550.1 YP_009106801.1 YP 009093042.1 NP 043712.1 YP_009093282.1 ADB 27491.1

YP 004072537.1 YP_009093440.1 $\mathrm{AE} \overline{\mathrm{X}} 37816.1$ YP 003735004.1 ADI 40219.1 ADI 40351.1 NP_039275.1 NP 043015.1 CAĀ 60276.1 ARK1 4872.1 YP 009367394.1 AAV̄80598.1 ABB81942.1 YP 009106261.1 YP 009106417.1 AIT̄95281.1 YP_009105929.1
LSETPPLNETSSSHPKENOVKGEENLTMAADINAVHTCLEHLLEGOGED--DTDUR LGRDRINSKLKSS-------TSSRQLSSKDIEFACYYLKKIERNQIL DD--DID ULKNR 667 LGRSRVDTKLENRAVGCSFLSCDSTQLMARDLELASLYLAGVKRKKLL DD--DID HLKNR 490 IGRHKLNQRLDLK-----ISQEFSSIIYDDIFAIIDYLITMSITKGG \$D--DID HLKNR 626 IGRFQINNRLNLK------LNNRIYTITYEDIFAILDCLVTLSISKTT $\$ D--D I D / H L K N R \quad 603$ IGRFKINNRLNLN------VPQQIQTITYEDIFAIIDSLIKLSISKTI \$D--DID ILKNR 600 IGRSKLNNRLNIQ------LSKRIVTITYQDIFAIIDKLITLSISKQI\$D--DID HLKNR 588 IGRSKLNNRLNIQ------LSKRIVTITYQDIFAIIDKLITLSISKQI \$D--DID HLKNR 588 IGRSKLNNRLDLN------LSTRITTITYEDIFAIIDQLIILATSKQV \$D--DID HLKNR 610 IGRIQLNKRLNIN------ISERYQTITYEDIFAITDKLLTLTISKTI AD--DIDHLKNR 602 VGRLKINNRLNLQ------LSERLQIITYEDIFAITDKLINLTISKAVPD--DIDHLKNR 609 VGRLKINNRLNLQ------LSERLQIITYEDIFAITDKLINLTISKAV PD--DID HLKNR 609 VGRVKMNHHLNLQ------LSERLQI ITYEDIFAITDKLINLTISKAV DD--DIDHLKNR 618 IGRLNLNKKLNLN------VPENEIFVLPQDILAAVDYLIKLKFGIGT D--DIDHLKNR 307 VGRRNMNRRLNLD-----IPQNNTELIPRDVLAATDHLIGMKEGTGI $\quad$ ILKNK 312 VGRRNMNRRLNLD------IPQNNTFLLPRDVLAATDHLIGMKFGTGI IDDDDMN HLKNK 312 LGRSK INKKLGLT------IAPDYTQLTGQDVLAACLFLMNLLQGLVL AD--DIDPLANR 449 LGRLRINQKLGLC------ISPDHTTLTAQDIFVACLFLMDLLQGLVT \$D--DIDPLKNR 525 LGREKINKKLGIS------IPLEKTILTSQDVLFACLFLMDLLQGLVI $\$$ D--DIDPLKNR 424 LGRLRLNQKLNLS------VNENITTITAQDLLFITNTLINLNYGVGKY D--DID NLKNR 380 LGRLRLNKKLGIS------VPLNQYILTQQDFLYATDYLIKLENGNGA D--DIDNLKNR 311 LGRLRLNNKLGIS------VPLNQYTLTPQDFLYATDYLIKLENGSGVID--DIDNLKNR 311 LGRLRLNNKLGIS------VPLNQYTLTPQDFLYATDYLIKLENGSGV 1 D--DID JLKNR 311 LGRLRLNKKLGIS------VPLHQYILTPQDFLYATDYLIKLENGNGV D--DIDNLKNR 311

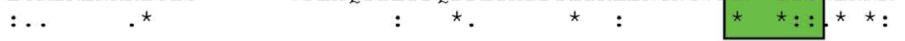

-FSLENETPERWLRTSKPVNNAFRE FESNPLSQYMDQTNPLAEITH KRRLSSMGPG IK 1138 VTSSHLFKILRSVITTKALNGALRE FGSNPLSQYMDQTNPLAEVTHKRRLSSLGPGCIS 904 -----QSISLKRVIDIKALDGAWRE FE SNPLSQYMDQTNPLAEVTHKRRVSCLGPG VVS 577 -------SPLILTSNVISTTVRE FG SSQLSYMDQTNPLASLTHKRRISGLGPGGED 709 -------SGQISSFNI IGATVRE F F S QLSQYMDQTNPLSSLTHRRRISGLGPGdLD 686 -- - ---SSQLSSFNIVGATVRE FGS $F$ QLSQYMDQTNPLSSLTHRRRISGLGPG FD 683 ------SSQLSSFNIVGATIRE FEASQLSQYMDQTNPLSALTH RRRISGLGPG FD 671 -------SSQLSSFNIVGATIRE FEA FQLSQYMDQTNPLSALTH RRRISGLGPG FD 671 -------SSQLSSFNI I GATIRE FG S SLSQYMDQTNPLSSLTHRRRISGLGPGQFD 693 -------STHLLSFNVVNATVKE FE SSQLSQYLDQTNPLSSLTH RRRVSGLGPGQFE 685 -------AGYLLSFNIVNATIRE HE S SQLSQYLDQTNPLSSLTH RRRISGLGPG FD 692 -------AGYLLSFNIVNATIRE FG S $\$$ QLSQYLDQTNPLSSLTH RRRISGLGPGQFD 692 -------SGYLLSFNIVNATIRE FG S SQLSQYLDQTNPLSSLTHRRRISGLGPG FD 701 -RLPTPKSLVTS----TPLIMTFKE FGSHPLSQFLDQTNPLTEIVHKRRLSSLGPG LT 397

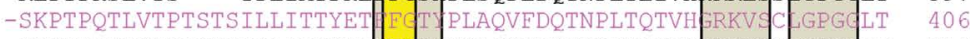
-SKPTPQTLVTPTSTSILLITTYET FETYPLAQVEDQTNPLTQTVHERKVSCLGPGCIT 406 ----LEPDILTNLFTVKPINQSLRE HET F PLSQLMDQTNALAELTH KRRLSSLGPGQ IN 537 -EKEQNSNFFSDLFTVKPLNQSFRE F FT PLSQLMDQTNALSELTH KRRLSSLGPG IN 639 ----QDSDSFSNLFSVKPLNQAFRD FGTNPLSQLMDQTNALSEITH KRRLSSLGPG IN 512 ----TKL-SIGSLINTKPINGALRE FGS PLSQFMDQTNPLAEITHKRRLSSLGPG GVN 467 ----KNF-TIKSLITTKPINGALRE FGSSQLSQFMDETNPLSEITH KRRVTSLGPGQIS 398 ----KKF-SIKSLITTKPINGALRE FES SQLSQFMDETNPLSEITH KRRVT SLGPGG IS 398 ----KKF-SIKSLITTKPINGALRE FE S SQLSQFMDETNPLSEITH KRRVT SLGPGCIS 398 ----KKL-SIKSLISTKPINGALRE FGS\$QLSQFMDETNPLSEITHKRRVT SLGPG IS 398

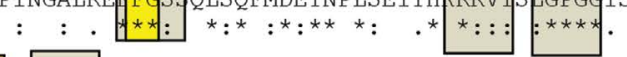

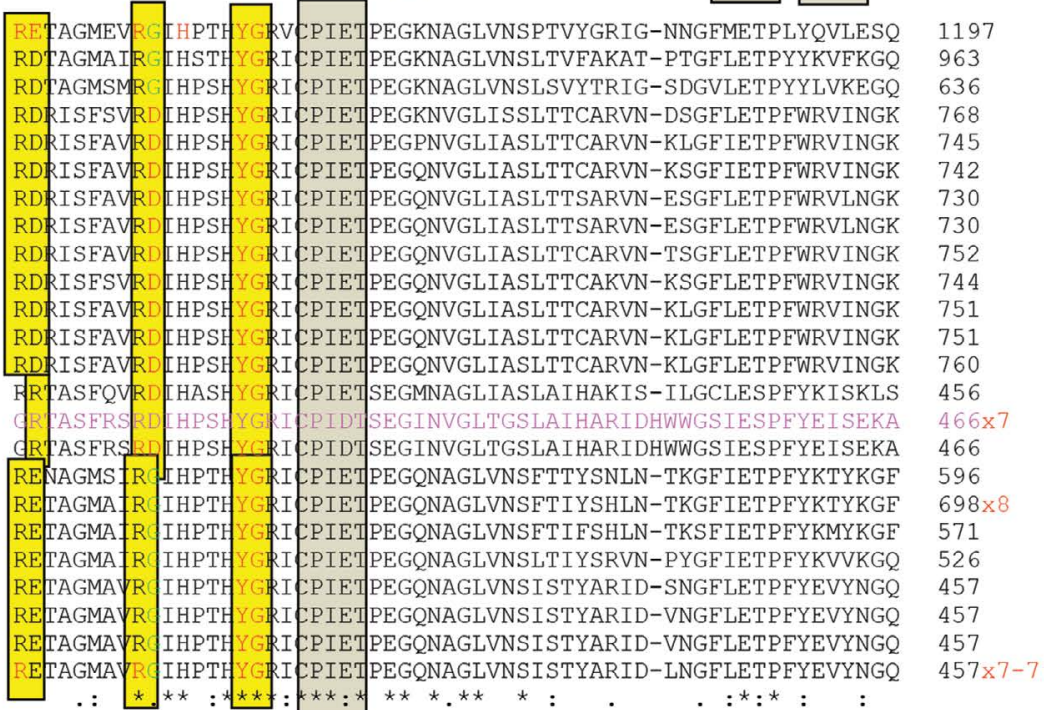


YP 009104799.1 YP-009106550.1 YP-009106801.1 YP-009093042.1 NP-043712.1 YP 009093282.1 $\mathrm{AD} \overline{\mathrm{B}} 27491.1$ YP 004072537.1 $\mathrm{YP}^{-} 009093440.1$ $\mathrm{AEX} 37816.1$ YP_003735004.1 ADI 40219.1 ADI 40351.1 NP 039275.1 NP 043015.1 CĀ̄ 60276.1 ARK14872.1 YP 009367394.1 AAV80598.1 ABB81942.1 YP 009106261.1 YP 009106417.1 AIT̄95281.1 YP_009105929.1

YP_009104799.1 YP 009106550.1 YP_009106801.1 YP_009093042.1 NP 043712.1 YP 009093282.1 $\mathrm{ADB} 27491.1$ YP 004072537.1 YP 009093440.1 AEX̄ 37816.1 YP 003735004.1 ADI 40219.1 ADI 40351.1 NP 039275.1 NP 043015.1 CĀ̄ 60276.1 ARK14872.1 YP 009367394.1 AAV 80598.1 ABB81942.1 YP 009106261.1 YP 009106417.1 AIT̄95281.1 YP_009105929.1

YP_009104799.1 YP 009106550.1 YP_009106801.1 YP_009093042.1 NP- 043712.1 YP 009093282.1 $\mathrm{AD} \overline{\mathrm{B}} 27491.1$ YP 004072537.1 YP 009093440.1 $\mathrm{AE} \overline{\mathrm{X}} 37816.1$ YP 003735004.1 ADI 40219.1 ADI 40351.1 NP 039275.1 NP 043015.1 CAA60276.1 ARK14872.1 YP 009367394.1 AĀ 80598.1 ABB81942.1 YP_009106261.1 YP 009106417.1 AIT̄95281.1 YP_009105929.1
RNRVGYKALSPVQTISIA RALIPF EEHDD RFKINCTALSPIOMTSTA TSL TPF FHDDANALM SNMOROAYPLLRPERP IVGTGLAS REQIQY IAISRIQMIS IA ESL IPF EHND A NRALM $\$$ NNMQRQA Y PLLTVEPP IVGTGLEG PSEIDFIAISTVQVVSVAASLIPF EEHDDA NRALM SNMORQSYPLLI PQRP I IGTGLEN PFOVDFISISPIOVVSVA TSLIPF EEHDDA NRALM S NMOROSVPLMLSOKP IVGTGLEN PFEVDFIAISPIQVVSVA ASL I PF EHDD ANRALM F NMQRQSVPLI LPQKP IVGTGLEN PSEVDF IAVSPIQVVSVAASLIPF EHDDA JRALM SNMQRQSYPLLLPQKP IVGTGLEN PSEVDFIAVSPIQVVSVAASLIPF EHDDA NRALM SNMQRQS PLLLPQKP IVGTGLEN PSEVDFIAVSP IQVVSVA ASLIPF FEHDDA NRALM SNMORQSYPLLLPQKP IVGTGLEN PSEVDFIA ISPVQVVSVAASLIPF EHDD A IRALM S SMORQSYPLLLPQKP IVGTGLEN PAEVDFIAISTVQVVSVAASLIPF EHDDA NRALM S SNMQRQS YPLIFPQKP IVGTGLEN PAEVDFIAISTVQVVSVAASLIPF EHDDANRALM SNMQRQSYPLIFPQKP IVGTGLEN PSEVDFIA ISTVQVVSVA ASLIPF EEHDDA NRALM \$NMQRQSYPLIFPQKP IVGTGLEN WEQVHLRS IFPLQY F SVGASL I PF EHNDA ARALM SNMQRQA Y PLLKPEKCIVGTGIES WEOTHVRSIFPFOYFSIG SSLIPE WEQIHVRS IFPFQYFSIGESLIPF EHNDA ARALM \$ SNMQRQAYPLSRSEKC IVGTGLER RKAINFTALSPIQMISVA TSLIPF EHDD $\$$ NRALM SNMQRQS I ATLRPSKP IVGTGLES RNEIHFTSMSPIOMISVA TSLIPFLEHND NRALM SNMOROAVPTIRPSKP IVGTGLE S RNEINFIAVSPIQMIS IA SSLIPF EHND \& NRALM \$ SNMQRQAYPTVRPSKP IVGTGLES REKIDYISVSPLQMISIA ESLIPF EHDD A NRALM $\$$ SNMQRQAYPLMTAEAP VVGTGLEC KNEIQY IGLSP IQMIS IA SSL I PF EEHDDA JRALM \$ SNMQRQAYPLMISEKP IVGTGLEF KNDIOY IGLSSIOMIS IA TSLIPF EHDD ANRALM SNMOROAYPLMI SEKP IVGTGLE F KNDIQY IGLSS IQMIS IA ESL IPF EHDD A NRALM $\$$ SNMQRQAY PLMISEKP IVGTGLEF KNDIOYIGLSSIOMISTA SLIPF EHDDA IRALM S SNMOROAYPLMISEKP IVGTGLF

$$
\begin{aligned}
& : \quad .:{ }^{*} . *::_{* * * *} * *: * \\
& * * * * *, * * * * * *=
\end{aligned}
$$$$
\text { - }
$$

RSNQETCLSQRPLVQEGDWVER GDFLAD SASSSGDLALGKNVRVAYMPWE GYNFFDATV RSNOETCLSHRPAVREGDWVOK GDLLAD SAS SKGELALGKNTLIAYI PWE GYNFHDAVY QSNQKTPLRHRPWVREGDWVQP GDLLAD SASQGGHLCLGRNVVVAYLPWI GYNF DAVY RSNQETCINQRPIVWKGEKVKS GQILAD \$PSMNGGELALGQNILVAYMPWH GYNE DAII RSNQETCINHRPIVWKGEKIKSGQILTD\&PGITNNELALGQNVLVAYMPWOGYNE' DAIT RSNQETCINQRP IVWKGEKIQSGQMLTD\&PGIANSELALGQNVLVAYMPWH GYNFE DAII RSNQETCINYRP IVWEGEQIKS FQMLTD \&PGI ISSELSLGQNVLVAYMPW GYNFE DAI RSNQETCINYRPIVWEGEQIKS GQMLTD\&PGI ISSELSLGQNVLVAYMPWQGYNE' DAI RSNQETCINHRP IVWEGQKVKS GQMLTD \&PGIVSSELSLGQNVLVGYMPWQGYNE DAI RSNQQTCINHRP I IWKGESIQS GQILTD \&PGINASELALGQNVLVGYMPW GYNF DDA RSNQQTCINHRP IVWKGESIKS GQI LTD\&PAITNSELALGQNLLVAYMPW GYNE LDAI RSNQQTCINHRP IVWKGESIKSGQILTD\&PAITNSELALGQNLLVAYMPWOGYNEHDAI RSNQQTCINHRPIVWKGEKIKS SQILTD\&PAITTSELALGQNVLVAYMPW GYNF DAII RSNNSTCMHQKPKVEKQKY IKKGQILAD\&AATANGELALGKNILVAYMPWE GYNF DAII RSNKNTCMHOKPRVPRGKSIKK FOILAE EAATVGGELALGKNVLVAYMPW RSNKNTCMHQKPRVPRGKS IKK GQI LAE \&AATVGGELALGKNVLVAYMPWE GYNFE DAVI RSNQGTYMIHRP IVYEGQWVEK GDILAD \$STSVQGELAIGQNILVGYTPWEGYNE EDAVI RSNQDTYMVHRPVVQEGQWVEK GDLLAD \$SASVQGELAIGQNILVGYTPWEGYNE DAVI RSNQDTYMIHRPLVQEGQWVEK GDI LAD \$SVSVQGELS I GQNILVGY I PWE GYNEHDAV RSNQDTSIMHRPVVREGEWVNR SDLLADWSTTVGGELSLGKNLLLAYMPWE GYNF DAII RSNQDTCVVQKPLVKEGEWIQKGDILAD \$IASIGGELSLGKNILIAYMPWE GYNE DDAII RSNQDTCVVQKPIVQEGDWVQK GDILAD \&IASIGGELALGKNILIAYMPWE GYNFEDAII RSNQDTCVVQKPIVQEGDWVQK FDILAD IAS I GGELALGKNILIAYMPWE GYNE DDAII RSNQDTCVVQKAVVKEGEWVQK SDILAD FIASIGGELALGKNILIAYMPWE GYNFE DAII

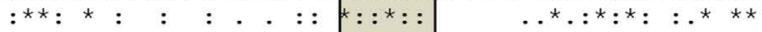

RHGNKGIVSIVLPRQDMPYL QDGSSVDMVLNPLGVPSRMNLGQIFE IL LA GSQLGCNF RHGNKGIVSVLLPRIDMPYL D GS IVDMI LNPLGVPSRMNVGQVFEOLLALA GVSLNOHY RHGNKGIVSTILPRQDMPYL DGTTVDMVLNPLGVPSRMNVGQVFESLELA FGFLQQRF

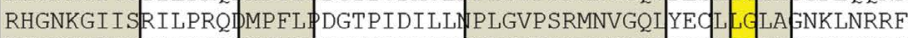
RHGNKGI ISRILPRQDMPF L DGTPVDI I LN PLGVPSRMNVGQI YEQLLGIA GHKLNRRF RHGNKGI I SRI LPRQDMPEL PDGTPVDI I LNPLGVPSRMNVGQI YEOLLCLA FHKLNRRF RHGNKGI ISRILARQDMPFL D GTPIDI I LNPLGVPSRMNVGQI YEQLLETAGHKLNRRF RHGNKGI ISRI LARQDMPE L D GTPIDI I LN PLGVPSRMNVGQI YEQLLG LA GHKLNRRE RHGNKGI I R ILPRQDMPFL DDGTPIDI I LN PLGVPSRMNVGQI YEOLLG LA GHKLNRRF RHGNKGI ISRILPRQDMPFL \$NGRPLDI I LNPLGVPSRMNVGQI YEOLE LA GDKLNCRF RHGNKGI ISRI LARQ WMPE L DDGTPIDI I LN PLGVPSRMNVGQI YEQLLE LA FDKLNSRE RHGNKG I ISRILARQDMPFL D DTP I D I I LNPLGVPSRMNVGQI YEOLLG LA GDKLNSRF RHGNKGI ISRILARQDMPFL DGTPIDVILWPLGVPSRMNVGQI YEOLLEA FDKLNSRF RHGNKGI ISKILPRQDMPFL $\$ D G T P I D M I L \$ P L G V P S R M N V G Q I F E Q L L E A$ FSFLHKNY RHGNKGIIS KILPRODMPYL DGAPVDMVEN PLGVPSRMNVGQIEESSE ADLLKKHY RHGNKGI I SKILPRQDMPYL DGAPVDMVFN PLGVPSRMNVGQIFESSLEA FDLLKKHY RHGNKGI ISNILPRQWMPYV \$DGTPLDIVLNPLGVPSRMNVGQIFEOLELA FYYLKQNY RHGNKGI I N N ILPRQDMPYL DGTPLDIVLN PLGVPSRMNVGQI IEOLLE IA GYYLKQNY RHGNKGI ISNILPRQDMPYL \$ DGTPLDIVLN PLGVPSRMNVGQ ILE QLECMA FYYLKQNF

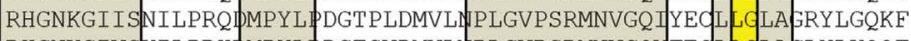
RHGNKGIVSKILPRHDMPYL D GT SVDMVLNPLGVPSRMNVGQVFEOLELA FLYLKQQF RHGNKGIVSKILPRHWMPYL D DTPIDMVLNPLGVPSRMNVGQVFEQLLLA FVYLNQQE

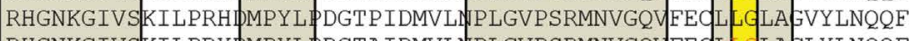

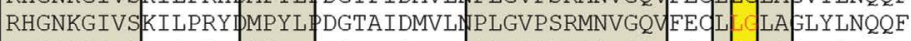

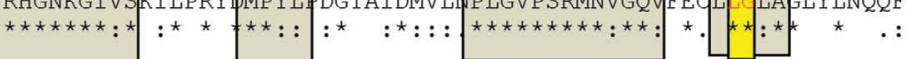


YP_009104799.1 YP_009106550.1 YP_009106801.1 YP_009093042.1 NP 043712.1 YP 009093282.1 ADB̄ 27491.1 YP 004072537.1 YP 009093440.1 AEX̄37816.1 YP 003735004.1 ADI40219.1 ADI40351.1 NP_039275.1 NP 043015.1 CĀ̄ 60276.1 ARK14872.1 YP_009367394.1 AĀ̄ 80598.1 ABB81942.1 YP_009106261.1 YP_009106417.1 AIT̄95281.1 YP_009105929.1

YP_009104799.1 YP 009106550.1 YP 009106801.1 YP 009093042.1 NP_0 043712.1 YP 009093282.1 ADB̄ 27491.1 YP_004072537.1 YP_009093440.1 AEX̄37816.1 YP_003735004.1 ADİ 0219.1

ADI40351.1

NP 039275.1

NP_043015.1

CĀ̄ 60276.1

ARK14872.1 YP_009367394.1 AAV̄80598. 1

ABB81942.1

YP 009106261.1

YP_009106417.1

AIT̄95281.1

YP_009105929.1
GQAY MLKLIHYVDHKIHARST GPYSLETRQPVRGRSR GGQR GEMEVWAVEGECAAYTL GTAY IIKLVH WVDEKIHARST GPYSL VTKQPLGGRSKH GGQR LGEMEVWALEGGE QAAYIL GRA YMLKLIHMVDEKIHGRSR GPYSL ITQQPVRGRGKQGGQR LGEMEVWALEGY GAAYTL GNAY I LKLIHLVDDKMHARATGPYSL VTQQPLGGKARH GGQR E GEMEVWALEGE GAAYTL GNAYMLKLIHLVDDKMHARATGPYSL TTQQPLGGKAQH GGQR E GEMEVWALEGEQAAFTL GNAYMLKLIH LVDKMHARATGPYSL VTQQPLGGKAQH GGQR E GEMEVWALE GECAAFTL GNAYMLKLIH LVDDKMHSRATGPYSL VTQQPLGGKAQH GGQR E GEMEVWALEGGECASFTL GNAY MLKLIHLVDDKMHSRATGPYSL VTQQPLGGKAQH GGQR A GEMEVWALEGEQASFTL GNAYMLKLIHLVDDKMHSRATGPYSLVTQQPLGGKAQHGGQR EGEMEVWALEGEEQASFTL GNAY MLKLIHLVDDKMHARAIGPYSL ITTQQPLRGKAQHGGQR FEEMEVWALE GF QAAFTL GNAYMLKLIHLVDDKIHARATGPYSLTTQQPLRGKAQHGGQR EE GEVVWALEGEQAAFTL GNA YMLKLIHLVDDKIHARAIGPYSL TTQQPLRGKAQHGGQRE GEMEVWALEGF QAAETL GNAY MLKLIH LVDDKIHARATGPYSL TTQQPLRGKAQH GGQR E GEMEVWALEGE AAAFTL GKAYMLKLIHQVDDKIHARSSGPYALVTQQPLRGRSRRGGQRUGEMEVWALEGGE QVAYIL GKSY ILKLI WVDEKIHGRSI GPYSI JTQQPVRGRAKOGGQR GEMEVWALA GE VAHIL GKSY ILKLIHOVDEKIHGRST GPYSL VTQQPVRGRAKOGGQR I GEMEVWALE GF QVVAHIL GIAYILKLIHLVDDKIHARST GPYSLVTQQQPLRGRAK GGGQRU GEMEVWALEGGEQAAYIL GVAY ILKLVHLVDDKIHARST GPYSL VTQQPLRGRSKQGGQRU GEMEVWALE GF QAAYIL GVAY ILKLIHLVDDKIHARST GPYSL VTQQPLRGRSKQGGQRVGEMEVWALEGE GAAYIL GQAUMLKLIH LVDEKIHARSI GPYSLVTQQPLRGRSKH GGQR IGEMEVWALEGE GAAYTL GQAYMLKLVHLVDEKIHARST GPYSLVTQQQPLRGRSKH GGQR IGEMEVWALEGECAAYTL GQAYMLKLVH LVDEKIHARST GPYSL VTQQPLRGRSKH GGQR UEMEVWALEGE GAAYTL GQAYMLKLVHLVDEKIHARST GPYSLVTQQPLRGRSKH GGQR IGEMEVWALEGGEAAYTL GQAY MLKLVHLVDEKIHARST GPYSLVTQQPLRGRSK GGQR UGEMEVWALEGE GAAYTL

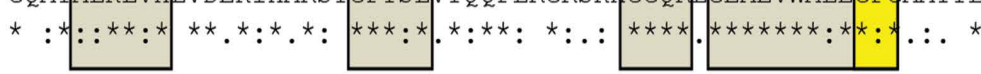

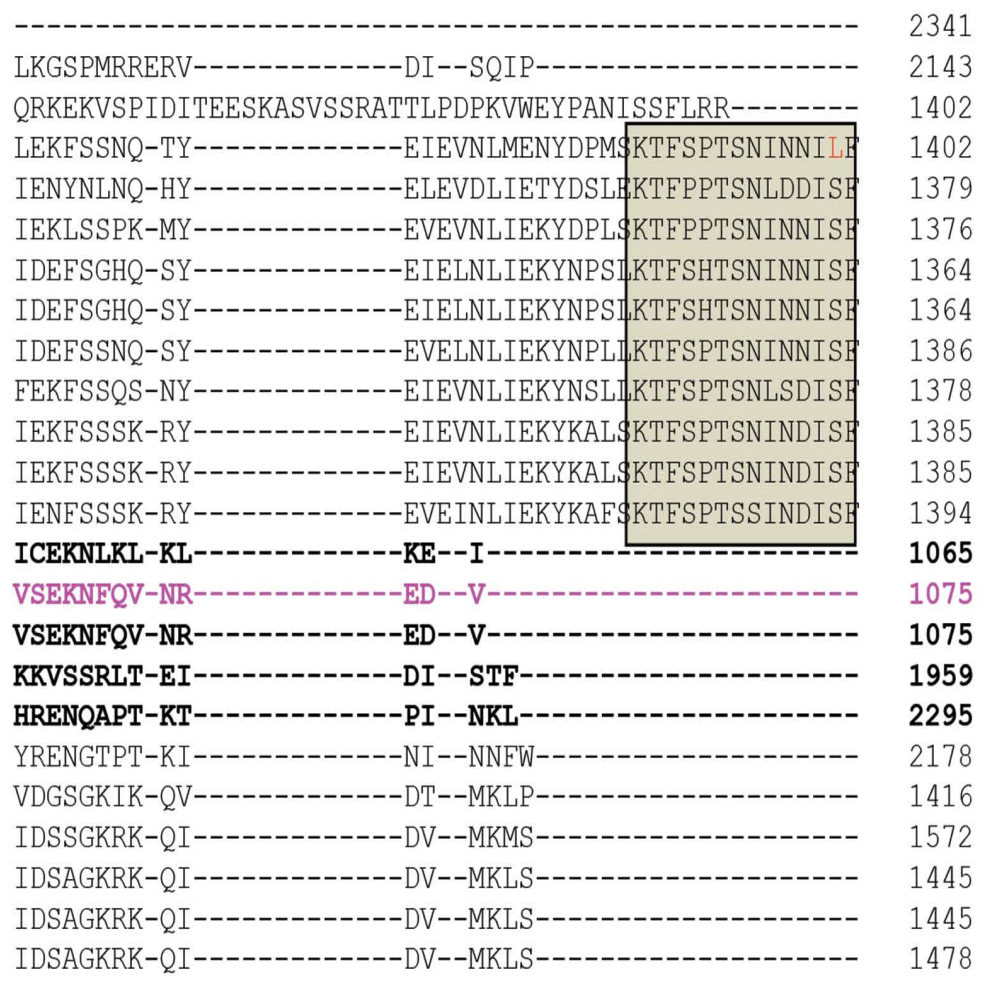

Figure 2. MSA of $\beta$ subunits of various chloroplast MSU RNAPs. 


\begin{tabular}{|c|}
\hline YP_009104799.1 Symbiochlorishandae \\
\hline YP_009106801.1 Elliptochlorisbilobata \\
\hline NP_043712.1 Odontellasinensis \\
\hline ADB27491.1 Thalassiosira oceanic \\
\hline YP_009093440.1 Thalassiosiraweissflogii \\
\hline YP_003735004.1 Durinskiabaltica \\
\hline ADI40351.1 Kryptoperidiniumfoliaceum \\
\hline NP_039275.1 Marchantiapolymorpha \\
\hline CAA60276.1 Zea mays \\
\hline YP_009367394.1 Trichosarcina mucosa \\
\hline YP_009106261.1 Interfilumterricola \\
\hline AIT95281.1 Geminella minor \\
\hline YP_009106550.1 Botryococcusbraunii \\
\hline YP_009093042.1 Rhizosoleniaimbricata \\
\hline YP_009093282.1 Cerataulina daemon \\
\hline YP_004072537.1 Thalassiosiraoceanica \\
\hline AEX37816.1 Ulnariaacus \\
\hline ADI40219.1 Durinskiabaltica \\
\hline ABB81942.1 Oltmannsiellopsisviridis \\
\hline NP_043015.1 Zea mays \\
\hline ARK14872.1 Chamaetrichon capsulatum \\
\hline AAV80598.1 Pseudendocloniumakinetum \\
\hline YP_009106417.1 Geminella minor \\
\hline YP_009105929.1 Gloeotilopsissterilis \\
\hline
\end{tabular}

by SDM or deletion mutants. Interestingly, a highly conserved region among the $\beta^{\prime}$ subunits from various sources is observed between amino acids 450 and 513, where the metal binding site is also located. Estimation of RNAP activity in the absence of this $\beta$ ' subunit will throw more light on the functional role of this subunit in catalysis. The sequence from Euglena shows few variant amino acids in the conserved region (marked in red). It should be noted that Euglena longa, a close relative of the photosynthetic model alga, Euglena gracilis, possesses an enigmatic non-photosynthetic plastid ${ }^{21}$.

\subsection{MSA of $\beta$ " Subunits MSU RNAPs from Chloroplasts}

Figure 4 shows the MSA and conserved motifs in $\beta$ " subunits of MSU RNAPs of chloroplasts. As found earlier, there are a large number of conserved motifs and some are found to be long stretches of conservations (highlighted). The Zea mays $\beta$ " subunit is used as the standard for numbering and highlighted in magenta. It is obvious from the Figure 4, that the $\beta$ " subunits are more conserved than the $\beta$ subunits of chloroplasts. It is interesting to note that the $\mathrm{Zn}$ binding motif, with 3 invariant Cs, that is found in all eubacterial elongation $\beta$ ' subunits is also found at the same distances in the $\beta$ " elongation subunits of all chloroplast MSU RNAPs (e.g., in E. coli $\beta$ ' the conserved Cs are found at amino acids 888, 895 and 898; in Zea mays $\beta$ " 296, 303 and 306) within the catalytic region of $\beta$ " subunits. X-ray crystallographic analysis has shown that this is the $\mathrm{Zn}$ binding motif in the thermophilic bacterium, Thermus aquaticus ${ }^{20}$. The completely conserved $\mathrm{Zn}$ binding motif in all eubacterial and chloroplast MSU RNAPs with absolute distance conservation of the Cs, suggests their possible role in the proof-reading activity. As seen in $\beta$ initiation subunits, the 'gatekeeper pair' YG and the catalytic R are found and are strictly conserved, including distance conservation in all 


\section{CLUSTAL O (1.2.4) MSA of $\beta$ ' subunits of various chloroplast MSU RNAP}

\begin{abstract}
SP|P0C506 | RPOC1 ORYSJ TDKPEKDGLFCERIEAHIKSRICACGNSRASG---AENEDERFCQKCGVEFVDSRIRRYQ 103 SP|A7M957|RPOC1_CUSRE TNKPEKDGLFCERIFG IKSGICACGNYRVIG---DEKEEPKFCEQCGVEFVDSRIRRYR 110 SP|B1X3M9|RPOC1 PAUCH TLKPEMIGLFCEKIFG SKDWECHCGKYKRVR---HR---GIVCERCGVEVTESRVRRHR 114 SP|Q1XDN6|RPOC1 ${ }^{-}$PYRYE TLKPEMIGLFCEKIFG VVDWECHCGKYKRFR---YK---GIVCERCGVEVTESRVRRHR 101 SP | P42080|RPOC1_CYAPA TLKPEMDGLFCEKIEGHVKDWECHCGKYKSIF---YR---GVICERCGVEITESQVRRHR 101 SP|Q4G3A6|RPOC1 EMIHU TLKPEMN GLFCERVFG HVNDECHCGKYKRIR---HK---GIVCERCGVEIIDSKVRRHR 98 SP 1019897 |RPOC1 CYACA TLKPEMDGLFCERIFGHIKNWECHCGKYKRIK---DK---GIICERCGVEVTESKVRRHR 103 SP | P51251 |RPOC1_PORPU TLKPEMDGLFCEKIFG HKDWECHCGKYKRFR---YK---GIVCERCGVEVTESRVRRHR 101 SP|Q6B8R7|RPOC1_GRATL TLKPEMDGLFCERIFG HKDWECHCGKYKRFR---YK---GVVCERCGVEVTESKVRRHR 101 SP |P56763|RPOC1 ARATH TNKPEKDGLFCERIFG IKSGICACGNYRVIG---DEKEDPKFCEQCGVEFVDSRIRRYQ 103 SP |P16024|RPOC1_MAIZE TDKPEK/GLECERI IKSGICACGNSRASV---RENEDERFCQKCGVEFVDSRIRRYQ 103 SP|Q85FM8|RPOC1 ADICA THKPERDGLFCERIFGHTKSGVCACGNCRSVN---DEGESSRECKHCGVEFTDSRVRRYR 103 SP 1085 CL 6 |RPOC1 ANTFO THKPEKDGLFCERIFGHIKSGICACGKYRSIE---NOREYSKICEQCGVEFTESRVRRYR 103 SP |P42079|RPOC1_SYNE7 TLKPEMIGLFCEKIEG HAKDWECHCGKYKRVR---HR---GIVCERCGVEVTESRVRRHR 101 SP|P11705|RPOC1_SPIOL TNKPEKDGLFCERIFGHIKSGICACGNYRVIG---DEKEDPKFCEQCGVEFVDSRIRRYQ 103 SP $|\mathrm{P} 14563| \mathrm{RPOC1}$ NOSCO TLKPEMDGLFCERIFGH-KDWECHCGKYKESV---IE---VLSVSAVVLEVTESRVRRHR 101 SP I Q2MIA9|RPOC1_SOLLC TNKPEKDGLFCERTEG IKSGICACGNYRVIG---DEKEDPKFCEQCGVEFVDSRIRRYQ 103 SP |Q6ENI3|RPOC1 ORYNI TDKPEKDGLFCERIFGHIKSRICACGNSRASG---AENEDERFCQKCGVEFVDSRIRRYQ 103 SP $\mid \mathrm{P} 46819$ |RPOC1 SINAL TNKPEKDGLFCERIFGHIKSGICACGNYRVIG---DEKGDPKFCEOCGVEFVDSRIRRYO 103 SP | P58131 |RPOC1_EUGLO KGKCIR GLFCEQIFGHTKKNTCRCGYYKNYKKSKKEKKHIKLCRICNVEITDPIIRNYR 101 SP 1078484 |RPOC1_GUITH TLKPEMI GLFCERIFG FVKDWCHCGKYKRVR---YK---GIVCERCGVEVAESKVRRHR 101 SP|Q2VEI5|RPOC1 SOLTU TNKPEKDGLFCERIE HIKSGICACGNYRVIG---DEKEDPKFCEQCGVEFVDSRIRRYQ 103 P|A6MVX3|RPOC1_RHDSA TLKPEMDGLFCERI E HVDWECHCGKYKRVR----YK---GIICERCGVEVAESKVRRHR 101 $\therefore \quad * \star \star \quad: \quad$ : $\quad$ * : :*.: :
\end{abstract}

SP|P0C506|RPOC1 ORYSJ LDSGSRGQPTRD--GHNKVYKSLSD IEGKE \$RFRETILGE VDYSGRSVIVVGPSLSLH 393

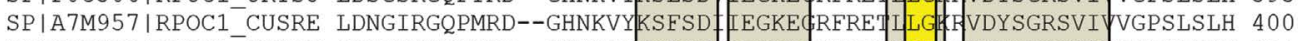
SP|B1X3M9|RPOC1 PAUCH IDNGRRGRTVVG--ANNRPI KSLSD IEGKQ_RFRQNILGIRVDYSGRSVIVVGPRLKIH 384 SP |Q1XDN6|RPOC1 ${ }^{-}$PYRYE MDNGRRGRTVVG--ANNRPI KSLSD IEGKQQRERQN LGHEVDYSGRSVIVVGPHLKLH 376 SP | P42080|RPOC1_CYAPA IDNGRRGRIVVG--AKNRPI KSLSD IEGKQ \&RERQN ILGE HVDYSGRSVIVVGLQLKLY 375 SP $\mid$ Q4G3A6 |RPOC1 EMIHU IDNGKRGRAVVG--LNNRPL KSLSD IEGKQ SP $1019897 \mid$ RPOC1 $^{-}$CYACA IDNGRRGRVVVG--ANNRAI KSLSN IEGKQ6RFRQNILGHRVDYSGRSVIVVGPDLKLN 372 SP | P51251 |RPOC1_PORPU MDNGRRGRTVVG--ANNRPL KSLSD _EGKQ\$RFRQN LLGE HVDYSGRSVI VVGPHLKLH 376

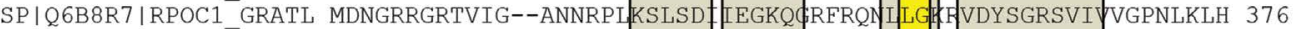
SP |P56763|RPOC1 ARATH LDNGIRGOPMRD--GHNKVYKSFSDVIEGKE\$RFRETILG HRVDSGRSVIVVGPSLSLH 393 SP|P16024|RPOC1_MAIZE LDSGSRGQPTRD--GHNKVY KSLSD EGKE REREI E

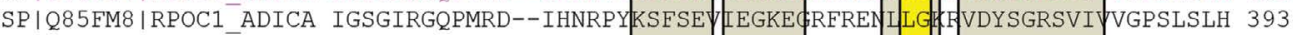
SP 1085 CL 6 |RPOC1 ANTFO IDNGIRGOPMRD--SHNRAYKSFSD|IEGKEGRFRENILGUEVDYSGRSVIVAGPYLPLH 393 SP |P42079/RPOC1_SYNE7 IDNGRRGRTVVG--ANNRPI KSLSD_IEGKQ\&RERQNILGHEVDYSGRSVI VVGPLKIH 370

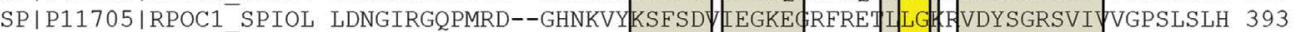

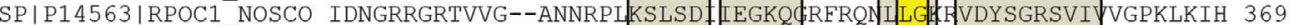
SP |Q2MIA9|RPOC1_SOLLC LDNGIRGQPMRD--GHNKVYKSFSD |IEGKE\&RFREI LG IRVDYSGRSVIVVGPSLSLH 393 SP |Q6ENI3|RPOC1-ORYNI LDSGSRGQPTRD--GHNKVYKSLSDVIEGKE\$RFRETLLGEHVDYSGRSVI) VGPSLSLH 393 SP |P46819|RPOC1 SINAL LDNGIRGQPMRD--GHNKVYKSFSDVIEGKEGRFRET LGHEVYSGRSVIVVGPSLSLH 401 SP | P58131 |RPOC1_EUGLO INNEKYKENKLGKIINNKKLKSITENIKGKE\$IIRENMLG RIVNFSGRSVI VVEPLNLN 348 SP $1078484 \mid$ RPOC1_GUITH IDNGRRGRTVMG--ANNRPIKSLSD IEGKQ\&RFRQN LGGKHDYSGRSVIVVGPQLELN 370 SP |Q2VEI5 |RPOC1 SOLTU LDNGIRGQPMRD--GHNKVYKSFSD|IEGKE $\$$ RERETLLGFRVDYSGRSVI)VGPSLSLH 399

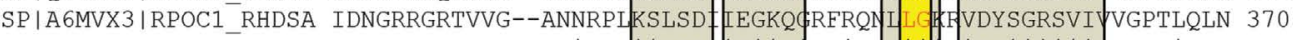
:. .

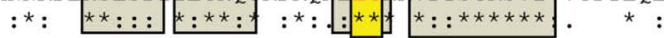

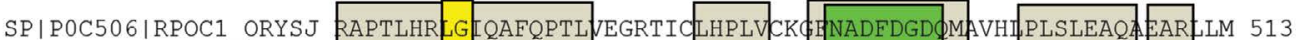
SP |A7M957|RPOC1_CUSRE RAPTLHRLGIQAFQPVLVEGHVLCLHPLVCK\$NNADFDGD\$MAVHYPLSLEAQAEAR_LM 520 SP |BIX3M9|RPOC1 ${ }^{-}$PAUCH RAPTLHRLGIOAFEPKLVDGRAIOLHPLVCPA HNADFDGDOMAVHVPLSIEAO FEAR ITM 504 RAPTLHRLGIQAFEPILVEGRAIKLHPLVCPA NADFDGDQMAVHYPLSLEAQAFARLLM 496 SP | P42080 IRPOC1_CYAPA RAPTLHR_SEQAFEPILVEGRAIOLHPLVCPA INADFDGD \$MAVHYPLSLEAQTEAR_LM 495 SP | Q4G3A6 | RPOC1 EMIHU RAPTLHRLG IQSFEPILVSGRAIR LHPLVCPA HNADFDGDQMAVHIPLSLEAQ\$EARLLM 484 SP |P51251|RPOC1_PORPU RAPTLHRLGIQAFEPILNEGRAIKLHPLVCPA INADFDGD\$MAVHYPLSLEAQAEAR_LM 496 SP|Q6B8R7|RPOC1_GRATL RAPTLHRLGIQAFEPILNEGRAIKLHPLVCPA HNADFDGDQMAVHYPLSLEAQAEARELM 496 SP|P16024|RPOC1_MAIZE SP | Q85FM8 | RPOC1 ADICA SP $\mid$ O85CL6 | RPOC1 ANTFO SP |P42079|RPOC1_SYNE7 SP | P11705 |RPOC1_SPIOL SP | P14563|RPOC1 NOSCO SP I Q2MIA9 | RPOC1_SOLLC SP|Q6ENI3|RPOC1 ORYNI SP|P46819|RPOC1 SINAL SP | P58131 |RPOC1 EUGLO SP|078484|RPOC1_GUITH SP|O2VEI5 |RPOC1 SOLTU SP |A6MVX3 | RPOC1_RHDSA 


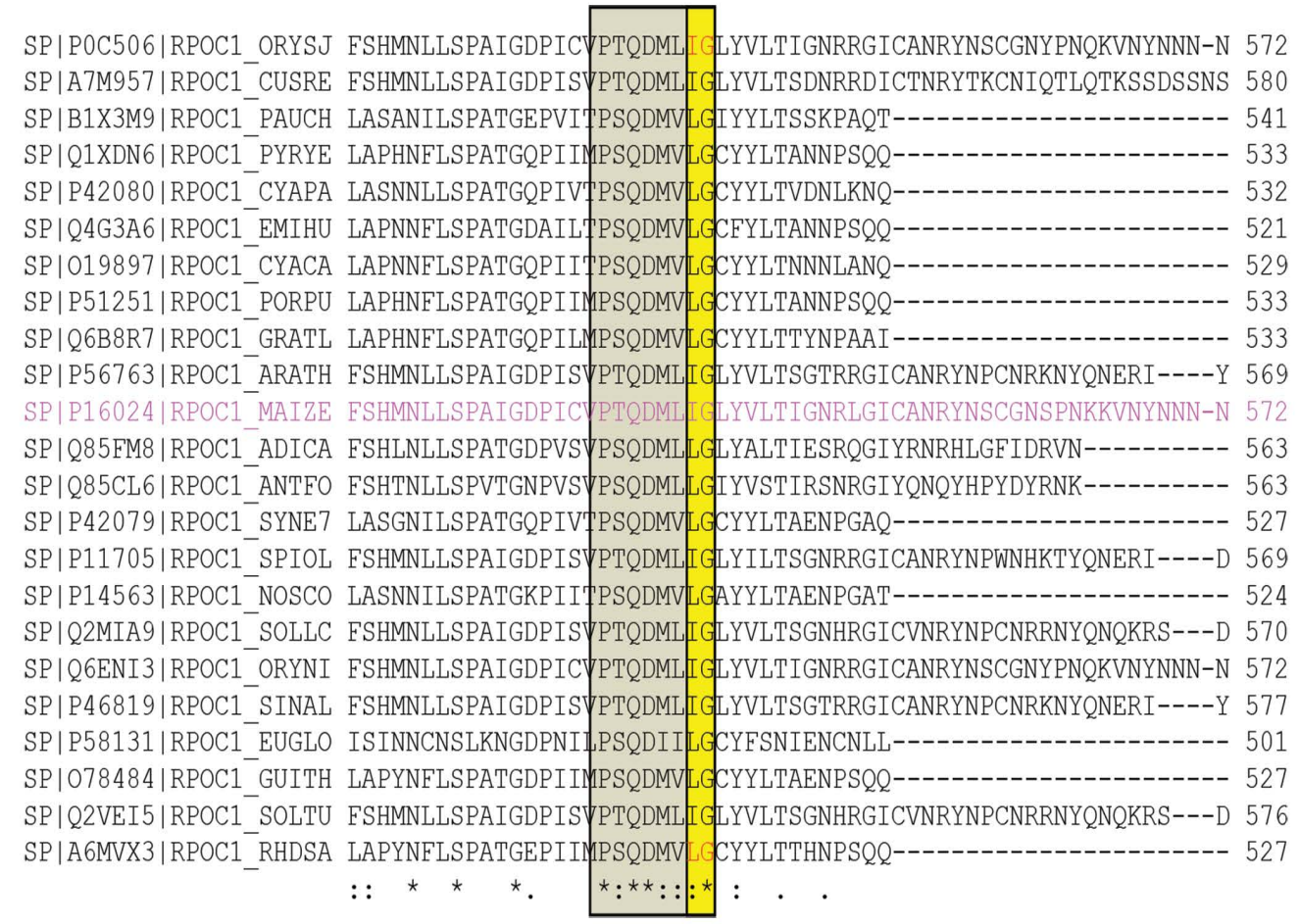

Figure 3. MSA of $\beta$ ' subunits of various chloroplast MSU RNAPs.

\begin{tabular}{|c|}
\hline SP|P0C506|RPOC1_ORYSJ Oryza sativa subsp.japonica \\
\hline SP|A7M957|RPOC1_CUSRE Cuscutareflexa \\
\hline SP|B1X3M9|RPOC1_PAUCH Paulinellachromatophora \\
\hline SP|Q1XDN6|RPOC1_PYRYE Pyropiayezoensis \\
\hline SP|P42080|RPOC1_CYAPA Cyanophoraparadoxa \\
\hline SP|Q4G3A6|RPOC1_EMIHU Emilianiahuxleyi \\
\hline SP|O19897|RPOC1_CYACA Cyanidium caldarium \\
\hline SP|P51251|RPOC1_PORPU Porphyrapurpurea \\
\hline SP|Q6B8R7|RPOC1_GRATL Gracilariatenuistipitata var. liui \\
\hline SP|P56763|RPOC1_ARATH Arabidopsis thaliana \\
\hline SP|P16024|RPOC1_MAIZE Zea mays \\
\hline SP|Q85FM8|RPOC1_ADICA Adiantum capillus-veneris \\
\hline SP|Q85CL6|RPOC1_ANTFO Anthoceros formosae \\
\hline SP|P42079|RPOC1_SYNE7 Synechococcus elongatus \\
\hline SP|P11705|RPOC1_SPIOL Spinacia oleracea \\
\hline SP|P14563|RPOC1_NOSCO Nostoc commune \\
\hline SP|Q2MIA9|RPOC1_SOLLC Solanum lycopersicum \\
\hline SP|Q6ENI3|RPOC1_ORYNI Oryza nivara \\
\hline SP|P46819|RPOC1_SINAL Sinapis alba \\
\hline SP|P58131|RPOC1_EUGLO Euglena longa \\
\hline SP|O78484|RPOC1_GUITH Guillardia theta \\
\hline SP|Q2VEI5|RPOC1_SOLTU Solanum tuberosum \\
\hline SP|A6MVX3|RPOC1_RHDSA Rhodomonassalina \\
\hline
\end{tabular}




\section{CLUSTAL O (1.2.4) MSA of $\boldsymbol{\beta}$ " subunits of various chloroplast MSU RNAP}

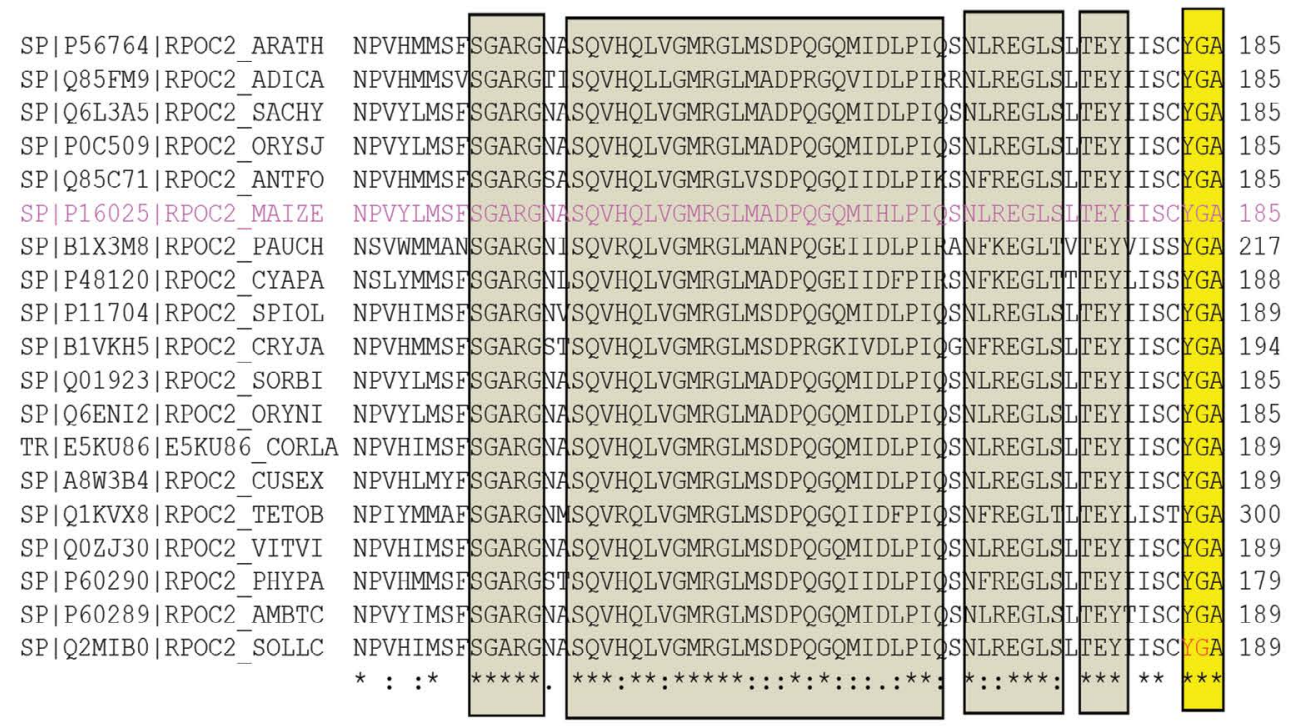

SP|P56764 |RPOC2 ARATH SP |Q85FM9 | RPOC2_ADICA SP IQ6L3A5 |RPOC2_SACHY SP |P0C509 | RPOC2_ORYSJ SP |Q85C71|RPOC2 ANTFO SP|P16025|RPOC2 MAIZE SP |B1X3M8|RPOC2 PAUCH SP |P48120 | RPOC2_CYAPA SP |P11704 | RPOC2 ${ }^{-}$SPIOL SP |B1VKH5 | RPOC2 CRYJA SPIQ01923|RPOC2 SORBI SPIQ6ENI2 | RPOC2_ORYNI TR |E5KU86 |E5KU86_CORLA SP |A8W3B4 | RPOC2_ CUSEX SP |Q1KVX8 | RPOC2_TETOB SP |Q0ZJ30|RPOC2 VITVI SP | P60290 | RPOC2 PHYPA SP|P60289|RPOC2 AMBTC SP |Q2MIBO |RPOC2 ${ }^{-}$SOLLC

SP|P56764|RPOC2 ARATH SP |Q85FM9 | RPOC2 ADICA SP IQ6L3A5 |RPOC2_SACHY SP |P0C509|RPOC2 ORYSJ SP IQ85C71 | RPOC2 ANTFO SP|P16025|RPOC2 MAIZE SP | B1X3M8 | RPOC2_PAUCH SP|P48120|RPOC2 CYAPA SP |P11704 |RPOC2_SPIOL SP|B1VKH5 | RPOC2 ${ }^{-}$CRYJA SPIQ01923|RPOC2 SORBI SP |Q6ENI2 | RPOC2_ORYNI TR|E5KU86 | E5KU8 $\overline{6}$ CORLA SP |A8W3B4 | RPOC2 CUSEX SP |Q1KVX8 | RPOC2 ${ }^{-}$TETOB SP IQOZJ30|RPOC2_VITVI SP|P60290|RPOC2 PHYPA SP | P60289 | RPOC2_AMBTC SP IQ2MIBO | RPOC2 ${ }^{-}$SOLLC

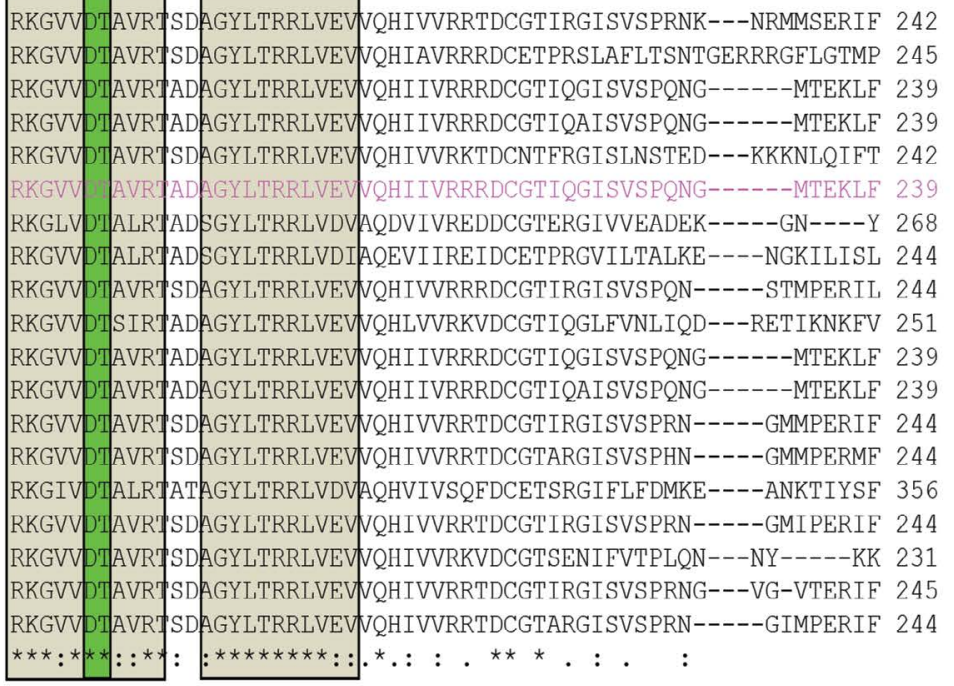

IQTLIGRVLADDIYIG---------SRCVAFRNQDLGIGLVNRLIT------FGTQSISI 288 HQGLVGRVLADHVYWD---------VRCIATRNQDISDGLASNLMA--------SSQPIHVR 290 VQTLIGRVLADDIYIG--------SRCIASRNQDIGIGLVNRE ITAFRAQPFRAQPIYI 291 VQTLIGRVLANDIYIG--------SRCIATRNQDIGIGLVNRE ITTFRAQPERAQPIYI 291 QQKLVGRVLADNLYIN---------ARCLAIRNQDINTNLIEKLIT-------LKTPLISI 288 VQTLIGRVLADDIYIG--------SRCIASRNQDIGIGLVNREITAFRAQPFRAQPIYI 291 GTKLVGRLAAQSVVVDNE-------NRVLVRRNREIDLITACRIEA------ADIPSVIVR 316 KDRLVGRVLLHNLYHPKT------HSLIAQKNESISVLLADDIIK------AGLKEVWI 292 IQTLIGRVLADDIYMG--------SRCIATRNQDIGVGLVNRFIT-------LRTQLISI 290 LQTLIGRVLADDVYIH---------GRCIATRNEDIGIKLANQLYY-------FQVQPIYI 297 VQTLIGRVLADDIYIG---------SRCIASRNQDIGIGLVNRFITAFRAQPFRAQPIYIR 291 VQTLIGRVLANDIYIG--------SRCIATRNQDIGIGLVNRFITTFRAQPFRAQPIYI 291 IQTLIGRVLADNIYMG--------PRCIGIRNQDIGIGLVNREIT------FQTQPISIR 290 IQTLIGRVLADDIYIG---------PRCIATRNQNIGVGLVNRFLN-------FRAEPILI 290 QNRLVGRVLAQDIFSTKEINKISDKKLIASRNQEIDSKLAFAISK-------VTKKALVR 409 IQTLIGRVLADDIYMG---------PRCIAIRNQDIGIGLVNRE IT-------FQAQTISIR 290 NNKLIGRILADNIYIN--------GRCIAIRNQDITTNLVISLIN------FQRKGIFIR 277 IQTLIGRVLANDVYMG--------LRCIATRNQDIGIGLVNREIT------SRAQPIYI R 291 SQTLIGRVLADDIYMG---------SRCIATRNQAIGIGLVNREIT------FRAQPISI 290×16 
SP|P56764|RPOC2 ARATH SP IQ85FM9|RPOC2 ADICA SPIQ6L3A5IRPOC2 SACHY SP | P0C509|RPOC2 ORYSJ SP IQ85C71|RPOC2 ANTFO SP|P16025|RPOC2 MAIZE SP|B1X3M8 |RPOC2 2 PAUCH SP|P48120|RPOC2 CYAPA SP|P1170 4 |RPOC2 SPIOL SP|B1VKH5|RPOC2 CRYJA SP I Q01923 |RPOC2 SORBI SPIQ6ENI2 |RPOC2 ORYNI TR|E5KU86|E5KU8 $\overline{6}$ _CORLA SP |A8W3B4 |RPOC2 CUSEX SP IQ1KVX8 |RPOC2 TETOB SP IQ0ZJ30|RPOC2 VITVI SP |P60290|RPOC2 PHYPA SP |P60289|RPOC2 AMBTC SP I Q2MIB0 |RPOC2 SOLLC

SP |P56764|RPOC2 ARATH SP IQ85FM9|RPOC2 ADICA SPIQ6L3A5|RPOC2_SACHY SP I P0C50 9 |RPOC2 ORYSJ SP I Q85C71 |RPOC2 ANTFO SP |P16025 |RPOC2 MAIZE SP |B1X3M8|RPOC2 PAUCH SP | P48120|RPOC2 CYAPA SP |P11704 |RPOC2 SPIOI SP | B1VKH5 |RPOC2 CRYJA SP IQ01923|RPOC2 SORBI SPIQ6ENI2 IRPOC2 ORYNI TR|E5KU86|E5KU8 $\overline{6}$ CORLA SP |A8W3B4 |RPOC2 CUSEX SP | Q1KVX8|RPOC2 TETOB SPIQ0ZJ30|RPOC2 VITVI SP |P60290|RPOC2 PHYPA SP | P60289|RPOC2 AMBTC SPIQ2MIB0|RPOC2_SOLLC

SP | P56764|RPOC2 ARATH SP IQ85FM9|RPOC2_ADICA SP IQ6L3A5 IRPOC2 SACHY SP | P0C509|RPOC2 ORYSJ SP I Q85C71 | RPOC2 ANTFO SP | P16025 |RPOC2 MAIZE SP |B1X3M8 |RPOC2_PAUCH SPIP48120|RPOC2 CYAPA SP | P11704 |RPOC2 SPIOI SP |B1VKH5 |RPOC2_CRYJA SPIO01923|RPOC2 SORBI SPIQ6ENI2 | RPOC2_ORYNI TR|E5KU8 6 |E5KU8 $\overline{6}$ CORLA SP |A8W3B4 IRPOC2 CUSEX SP IQ1KVX8|RPOC2 TETOB SPIQ0ZJ30|RPOC2_VITVI SP | P60290|RPOC2 PHYPA SP |P60289|RPOC2_AMBTC SP I Q2MIBO |RPOC2 SOLLC

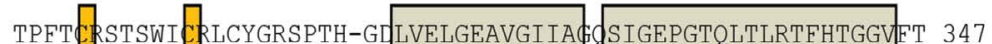
SPLTK.SIFWI EEFCYGWSLAH-CN LVELGEAVGIIAф SIGEPGTQLTLRTFHTGGVET 349 TPFTCRSTSWICDLCYGRSPTH-GDLVELGEAVGIIA\& SIGEPGTQLTLRTEHTGGVET 350 TPFTERSTSWIC QLCYGRSSTH-GD LVELGEAVGVIA \&SIGEPGTQLTTRTTFHTGGVET 350 SPLTC.SMLWI EDLCYGWSLTHYGLLVELGEAVGIIA\& SSIGEPGTQLTLRTFHTGGVET 348 TPET SSTSWI DLCYGRSPTH-GL LVELGEAVGIIA A SIGEPGTQLTLRTEHTGGY 350 SPLTC AAARSVERKCYGWALAH-NA LVDLGEAVGIIAAdSIGEPGTQLTMRTFHTGGVST 375 SPLTCKATRSVEQYCYGNNLAH-GELVELGEAVGIIAAdSIGEPGTQLTMRTFHTGGVTT 351 TPFTCRSASWICRLCYGRSPTH-GQLVELGEAVGIIA\& SIGEPGTQLTLRTFHTGGVET 349 TPFT C KIYWI CQLCYGRNTTH-SN LIELGEAVGIIA\&OSIGEPGTQLTLRTFHTGGVT 356 TPFTC.STSWI CLCYGRSPTH-GLLVELGEAVGIIA\&SSIGEPGTQLTLRTFHTGGVET 350 TPFTERSTSWI EDLCYGRSSTH-GDLVET,GEAVGVIA\&OSIGEPGTQLTTRTFHTGGVFT 350 TPFT CRSTFWI ERLCYGRSPTH-GLLVELGEAVGIIA\& SIGEPGTQLTLRTFHTGGVET 349 TPLTC KSTSWIC KLCYGRSPTH-GLLVELGEAVGIIA\& OSIGEPGTOLTLRTFHTGGVT 349 SPLTE ETPRLIEQLCYGWSLSQ-GI LVSVGEAVGVIAA SSIGEPGTQLTMRTFHTGGVEA 468 TPFTKKSTSWI CRLCYGRSPTH-GDLVELGEAVGIIA\& SIGEPGTQLTLRTFHTGGVET 349 SPLI \& KMLWI E LCYGWSLTH-GN LIELGEAVGIIA @SIGEPGTQLTLRTEHTGGVET 336 SPFTCRSTSWI CQLCYGRSTTH-GN LVELGEAVGIIA\& SIGEPGTQLTLRTFHTGGVET 350 TPFT ERSTSWI ERLCYGRSPTH-GDLVELGEAVGIIA\& SIGEPGTQLTLRTFHTGGVT 349 $: *$ : $::$ : $: * * * \quad:: \quad *:: * * * * *: * * * * * * * * * * * * *: * * * * * * *$ :

LPGELIGLLRAERTGRALEE----AICYRAVILGI RASLNTQSFIS ASFQETARVLAK 1294 LPGELVELSQAERINRVLKK----SIFYEPIVLGMTRASLSTTSFLAA ASFQETTRVLAK 1326 LPGELIGLLRAERAGRALDE----SIYYRAII LGI RASLNTQSFISEASFQETARVLAK 1443 SPGELIGLLRAERAGRALDE----SIYYRAIILGITRVSLNTOSFISEASFQETARVLAK 1424 LPGETIESSRAQRMNRVLEE----SVLYYKPIT LGT TKASLNTQSFISAASFQETTRVLAK 1349 LPGELI GLLRAERAGRALDE----SIYYRAIILGI RASLNTQSEIS A ASEQETARVIAK 1436 LPGELIELRHVEKVNKAMFMTSSSPAVFIPELLGITKASLNTDSFISA ASFQETTRVLTE 1233 LPNELIEFQQIEKMNEKFSTSNGQLASYTPIILGI TSSLNTQSFISA ASFQETTRVLAK 1192 LPGELI GLFRAERTGRALEE----AICYRATI LGI RASLNTQSFISEASFQETARVLAK 1287 LPGELVGLSRVQRMDRALEK----RINYRTIILGMTNASLNTQSFLSEASFQETAQVLAK 1092 LPGELI GLLRAERAGRALDE----SIYYRAIILGI RASLNTQSFISA ASFQETARVLAK 1429 SPGELI GLLRAERAGRALDE----SIYYRAIILGITRVSLNTQSFISEASEQETARVLAK 1424 SPGELIGLLRAERTGRALEE----AICYRAIL LGI TKASLNTQSFISEASEQETARVLAK 1291 SPGELIGLLRAERTGRALEE----PIHYRSV HGI KASLNTQSFISAASFQETARVLSK 1306 FPGELVNLDLVERINKFLLV----KIRYEPVVLGITRSSLEVDSFLSA SSFQQTTKILAL 2516 SPGELI GLLRAERTGRAFEE----AICYRTII LGITRASLNTQSFISA ASFQETARVLAK 1303 LPGELIEFARAKRMNRALEE----VIPYKPVILLGI KASLNTQSFISEASFQETTRVLAK 1250 SPRELIGLLRAERIGRALED----DICYRAIILGIXRASLNTQSFISEASFQETTRVLAK 1292 SPGETIGLLRAERMGRALEE----AICYRVVI LGT TRASLNTQSFISAASFQFTARVLAK 1303 $\star \star \star *: \quad:: \ldots: \quad: \quad * \star: \dashv . \star \star \ldots \star \star::::^{\star \star \star}: \star:::^{\star}:$

AALRGRIDWLKGLKENVVL GVIPA GTGFNKGLVHCSRQHTNIILEKKTKNLALFEGDMR 1354 AALRGRIDWLKGLKENVVI DSSPPVGTGSPEIYCQLNI-NKE------KESRLASGGSK 1378 AALRGRIDWLKGLKENVVL GIIPVGTGE-QKFVHRSPQDKNLYFE--IQKKNLFASEMR 1500 AALRGRIDWLKGLKENVVL GGIIPY GTGF-QKFVHRYPQNKNLYFE--IQKKKLFASEMR 1481 AALRGRIDWLKGLKENVIF \&GVISA GTGCQEVV-WQVILEKRKETYSKRKKNKLFSGRVR 1408 AALRGRIDH KGLKENVVL GIIPY GTCE-QKEVHRSPQDKNLYLE--IQKKNLFASEMR 1493 AAIEGKTDYLRGLKENVI I \&LIPAGTGESGEEE--------ELRS----------EAGPHP 1277 AAVEGKIDQLRGLKENVII \&NLIPAGTGESAYND------NAVF--------QNEDIE 1236 AALRGRIDWLKGLKENVVL GMIPV GTGF-KGFVHHSSQHKDIPLK--TKKQNLFEGEMG 1344 SALQGRIDWLKGLKENVI L GGMIPYGTGENR-LVKRSKMN-------SRTSQKSLF INKVE 1145 AALRGRIDWLKGLKENVVL GIIPYGTGF-QKFVHRSPQDKNLYFE--IKKKNLFASEMR 1486 AALRGRIDWLKGLKENVVL GGI IPYGTGF-QKFVHRYPQDKNLYFE--IQKKKLFASEMR 1481 AALRGRIDWLKGLKENVVL GMIPV GTGE-KGLAPRSRQHNNIPLE--TKKKNFFEGEMR 1348 AALGGRIDWLKGLKENVVL GVIPA GTGE-RGLVDPSKQYKTIPLK-----TNLFEGGMR 1360 AS ISRKKD FLKGLKENILV \&NLMP SGTGYMVL---RKNL------------------ 2552 AALWGRIDWLKGLKENVVL \$GMIPYGTGE-KGLVHRSRQHNNIPLEMETKKNNLFEREMR 1362 AALRGRIDWLKGLKENVIL GIIPT GTGCEEVL-WQITLEKQKNILLKKNKSKLFHNKVK 1309 AALRSRIDWLKGLKENVVL GGMIPYGTGE-KGFVHHSREHNNISLE--IKKKNLFDGKMR 1349 AALRGRIDWLKGLKENVVL GVIPYGTGE-KGLVHPSKQHNNIPLE--TKKTNLEEGEMR 1360 $::: \quad * *: * * * *::: \quad: * * *$

Figure 4. MSA of $\beta$ " subunits of various chloroplast MSU RNAPs. 


\begin{tabular}{|c|}
\hline SP|P56764|RPOC2_ARATH Arabidopsis thaliana \\
\hline SP|Q85FM9|RPOC2_ADICA Adiantum capillus-veneris \\
\hline SP|Q6L3A5|RPOC2_SACHY Saccharum hybrid \\
\hline SP|P0C509|RPOC2_ORYSJ Oryza sativa subsp. japonica \\
\hline SP|Q85C71|RPOC2_ANTFO Anthocerosformosae \\
\hline SP|P16025|RPOC2_MAIZE Zea mays \\
\hline SP|B1X3M8|RPOC2_PAUCH Paulinellachromatophore \\
\hline SP|P48120|RPOC2_CYAPA Cyanophoraparadoxa \\
\hline SP|P11704|RPOC2_SPIOL Spinacia oleracea \\
\hline SP|B1VKH5|RPOC2_CRYJA Cryptomeria japonica \\
\hline SP|Q01923|RPOC2_SORBI Sorghum bicolor \\
\hline SP|Q6ENI2|RPOC2_ORYNI Oryza nivara \\
\hline TR|E5KU86|E5KU86_CORLA Corynocarpuslaevigatus \\
\hline SP|A8W3B4|RPOC2_CUSEX Cuscutaexaltata \\
\hline SP|Q1KVX8|RPOC2_TETOB Tetradesmus obliquus \\
\hline SP|Q0ZJ30|RPOC2_VITVI Vitis vinifera \\
\hline SP|P60290|RPOC2_PHYPA Physcomitrella patens \\
\hline SP|P60289|RPOC2_AMBTC Amborellatrichopoda \\
\hline SP|Q2MIB0|RPOC2_SOLLC Solanum lycopersicu \\
\hline
\end{tabular}

the chloroplast $\beta$ " subunits. However, in the $\beta$ " elongation subunit, the catalytic R and the YG pair distance is maintained at 16 amino acids, almost double the distance as compared to the Binitiation subunits. This is in close agreement with the eubacterial elongation subunits. That is, in these $\beta$ " subunits the catalytic $\mathrm{R}$ is placed at $16^{\text {th }}$ position as found in $\beta^{\prime}$ subunits of eubacteria and hence suggests that the eubacterial $\beta$ ' and chloroplast $\beta$ " subunits are very similar in their catalytic regions and in distance conservations. This strongly suggests that the DNA polymerases, SSU and MSU RNAPs might use a similar set of amino acids for template, substrate binding and catalysis establishing a structure-function relationship among the DNA polymerases and RNAPs. The completely conserved $\mathrm{R}$ found downstream from the catalytic $R$ at $-7 / 8$ position and at +1 from the $Y G$ pair may play a role in NTP selection as suggested for other MSU RNAPs (Table 1). Another interesting observation is that the elongation subunits of eubacterial and chloroplast RNAPs possess two YG pairs, suggesting that the two YG pairs might be possibly recognizing both the strands and slide during the transcription process as seen in some viral polymerases 5 . However, the DNA polymerases and most of the SSU RNAPs use only one YG pair. In $\beta$ " also there are two conserved motifs at the C-terminal region (about 12 amino acids in length) as seen in other $\beta$ and $\beta$ ' subunits. However, the completely conserved metal binding motif - NADFDGDQMA - found in all $\beta$ ' subunits, is conspicuously absent in the $\beta$ " subunits confirming that the active site regions are split between the $\beta$ ' and $\beta$ " subunits in chloroplast MSU RNAPs. This is in contrast to the eubacterial $\beta$ ' elongation subunits, where both are located in the same subunit. These results suggest that the $\beta$ ' subunit harbouring the metal binding site may be also essential for the elongation process in chloroplast MSU RNAPs.

\section{Mix and Match Analysis}

\subsection{MSA of the $\beta$ Subunits of Chloroplast and Eubacterial MSU RNAP}

Only the initiation subunits $\beta$ of eubacteria and chloroplasts and the elongation subunits $\beta^{\prime}$ and $\beta^{\prime \prime}$ subunits of eubacteria and chloroplasts, respectively, were analyzed for their conserved motifs by this method. 


\section{CLUSTAL O (1.2.4) MSA of the $\boldsymbol{\beta}$ subunits of eubacteria and chloroplasts (Mix and Match analysis)}

SP|B4EYU9|RPOB PROMH SP|A7FNT3|RPOB YERP3 SP|Q1C1U1|RPOB YERPA SP|A8G8E7|RPOB_SERP5 SP|C5BHE3|RPOB_EDWI9 SP $|A 7 M Q Q 9|$ RPOB CROS8 Sp|B5XYF5|RPOB KLEP3 SP |P0A8V2 |RPOB ECOLI sp |Q31U10|RPOB SHIBS SP|Q32AF9|RPOB SHIDS SP|B5RFK1|RPOB_SALG2 Sp |B5BJQ3|RPOB SALPK SP|P06173|RPOB SALTY SP | Q9MTM5 | RPOB OENEH SP | Q50A72 |RPOB ACOGR SP IQOG9X0|RPOB_DAUCA tr|G1CC85|G1CC85 SOLTU splQ09G54|RPOB PLAOC

Sp |B4EYU9 | RPOB_PROMH SP |A7FNI3 |RPOB YERP3 SP IQ1C1U1|RPOB_YERPA sp|A8G8E7|RPOB SERP5 sp/C5BHE3|RPOB_EDWI9 sp |A7MQQ9 $\mid$ RPOB CROS8 SP |B5XYF5 |RPOB KLEP3 SP |POA8V2/RPOB ECOLI sp|Q31U10|RPOB SHIBS sp |Q32AF9|RPOB_SHIDS sp|B5RFK1|RPOB SALG2 sp |B5BJQ3 |RPOB_SALPK sp|P06173|RPOB_SALTY SP | Q9MTM5 |RPOB OENEH sp | Q50A72 | RPOB ACOGR Sp |Q0G9X0|RPOB DAUCA tr|G1CC85|G1CC85_SOLTU SP/Q09G54 |RPOB PLAOC

SP|B4EYU9|RPOB PROMH SP |A7FNI3 |RPOB YERP3 SP IQ1C1U1|RPOB YERPA SP|A8G8E7|RPOB SERP5 Sp|C5BHE3|RPOB EDWI9 sp |A7MQQ9 |RPOB_CROS8 sp|B5XYF5 |RPOB KLEP3 Sp | P0A8V2 |RPOB_ECOLI sp|Q31U10|RPOB SHIBS sp|Q32AF9|RPOB SHIDS sp|B5RFK1|RPOB SALG2 sp|B5BJQ3|RPOB SALPK sp|P06173|RPOB_SALTY SP | Q 29MTM5 |RPOB OENEH SP I 250A72 |RPOB_ACOGR SP|Q0G9X0|RPOB DAUCA $\operatorname{tr} \mid$ G1CC85|G1CC85 SOLTU sp/Q09G54|RPOB_PLAOC
MVYSYTEKKRIRKDFG-KRPQVLDVPYLLS LQLDSHEKFIEQDPDGQNGLEAAFRSVFPI 59 MVYSYTEKKRRIRKDFG-KRPQVLDTPYLISS IQLDSA QKFTEODPEGQHGLEAAFRSVFPI 59 MVYSYTEKKRTRKDFG-KRPQVLDTPYT,TS TQT,DSH QKFTF,ODPEGQHGTEAAAFRSVFPI 59 MVYSYTEKKRIRKDFG-KRPQVLDIPYLIS IQLDSH QKEIEQDPEGQHGLEAAFRSVFPI 59 MVYSYTEKKRIRKDFG-KRPQVLDVPYLIS IQLDSHOKFIEODPEGQYGLEAAFRSVFPI 59 MVYSYTEKKRIRKDFG-KRPQVLDVPYLLS IQLDSH QKEIEQDPEGQYGLEAAFRSVFPI 59 MVYSYTEKKRIRKDFG-KRPQVLDIPYLLS LQLDSHQKFIEODPEGQYGLEAAFRSVFPI 59 MVYSYTEKKRIRKDFG-KRPQVLDVPYLIS QLDS O EFIEOPPEGQYGLEAAFRSVFPI 59 MVYSYTEKKRIRKDFG-KRPQVLDVPYLLS IQLDSHQKEIEQDPEGQYGLEAAFRSVFPI 59 MVYSYTEKKRIRKDFG-KRPQVLDVPYLIS IQLDSHQKFIEQDPEGQYGLEAAFRSVFPI 59 MVYSYTEKKRIRKDFG-KRPQVLDVPYLLS EQLDSHQ KFIEOPPEGQCGLEAAFRSVFPI 59 MVYSYTEKKRIRKDFG-KRPQVLDVPYLLS IQLDSHOKFIEQDPEGQYGLEAAFRSVFPI 59 MVYSYTEKKRIRKDFG-KRPQVLDVPYLLSIQLDSH QKFIEODPEGQYGLEAAFRSVFPI 59

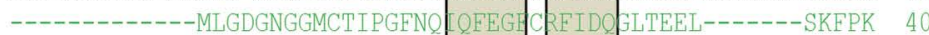

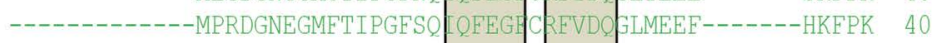
----------MLRDGNEGMSTIPGENQ OFEGH CEMDO SLTEEL------YKFPK 40 --------MLGDGNEGISTIPGENQ QEEGH C REIDOEVTEEL------YKFPK 40

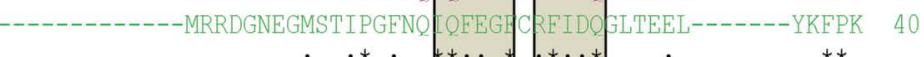

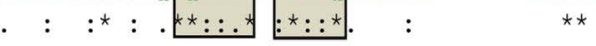

QSYSGNA----ELQYVSYRLGEPVEPVKECQIRG VTYSAPLRVKLRLVIYEREAPEGTVK 115 QSYSGNS----ELQYVSYRLGEPVEDVKECQIRGVTYSAPLRVKLRLVIYEREAPEGTVK 115 QSYSGNS----ELQYVSYRLGEPVE PVKECQIRGVTYSAPLRVKLRLVIYEREAPEGTVK 115 QSYSGNS----ELQYVSYRLGEPVEDVKECQIRGVTESAPLRVKLRLVIYEREAPEGTVK 115 QSYSGNS----ELQYVSYRLGEPVEDVKECQIRGVTYSAPLRVKLRLVIYEREAPEGTVK 115 QSYSGNS----ELQYVSYRLGEPVFDVECQIRGVTYSAPLRVKLRLVIYEREAPEGTVK 115 QSYSGNS----ELQYVSYRLGEPVEDVKECQIRGVTYSAPLRVKLRLVIYEREAPEGTVK 115 QSYSGNS----ELQYVSYRLE EPVE DVQECQIR VTYSA PLRVKLRLVIYEREAPEGTVK 115 QSYSGNS----ELQYVSYRLGEPVEPVQECQIRGVTYSAPLRVKLRLVIYEREAPEGTVK 115 QSYSGNS----ELQYVSYRLGEPVEDVQECQIRGVTYSAPLRVKLRLVIYEREAPEGTVK 115 QSYSGNS----ELQYVSYRLGEPVEDVQECQIRGVTYSAPLRVKLRLVIYEREAPEGTVK 115 QSYSGNS----ELQYVSYRLGEPVEDVQECQIRGVTYSAPLRVKLRLVIYEREAPEGTVK 115 QSYSGNS----ELQYVSYRLGEPVEDVQECQIRGVTYSAPLRVKLRLVIYEREAPEGTVK 115 IEDDTDQEIEFQLFVETYHLVELIERDAVYEI TYSSELYISAGLI-------WKSSR 93 IED-TDQEIEEQLEVERYQLVEPL KERDAVYES TYSSELYVPAGLI-------WKTGR 92 IED-TDQEIEFQLFVETYQLVEPL KERDAVYES LTYSSEFYISAGLI-------WKTSR 92 IED-TDQEIEFQLFVETYQLVEPL KERDAVYES TYSSELYYVSAGLI-------WKNSR 92 IED-TDQEIEEQLFVETYQLVEPL KERDAVYES TYSSEVYVPAGLI------WKPGR 92

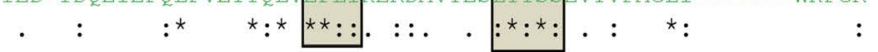

DIKEQEVYMGEIPLMTDNGT VING TERVIVSQLHASPGVFHDSDKGKTHSSGKVLYNAR 175 DIKEQEVYMGEIPLMTENGT EVINGTERVIVSQLHPSPGVFEDSDKGKTHSSGKVLYNAR 175 DIKEQEVYMGEIPLMTENGT EINGTERVIVSQLHP SPGVF DSDKGKTHSSGKVLYNAR 175 DIKEQEVYMGEIPLMTENGTE VINGTERVIVISQLHA SPGVFEDSDKGKTHSSGKVLYNAR 175 DIKEQEVYMGEIPLMTENGT EVING TERVIVSQLHRSSPGVFHDSDKGKTHSSGKVLYNAR 175 DIKEQEVYMGEIPLMTDNGT VING TERVIVSQLHA SPGVF DSDKGKTHSSGKVLYNAR 175 DIKEQEVYMGEIPLMTDNGT VINGTERVIVISQLHASPGVF DSDKGKTHSSGKVLYNAR 175 DIKEQ YYMGEIPLM TDNGT VINA TE RVIV SQLHR SPGVE DSDKGKTHSSGKVLYNAR 175 DIKEQEVYMGEIPLMTDNGTEVINGTERVIVSQLHPSSPGVFDSDKGKTHSSGKVLYNAR 175 DIKEQEVYMGEIPLMTDNGT VINGTERVIVSQLHRSPGVFHDSDKGKTHSSGKVLYNAR 175 DIKEQEVYMGEIPLMTDNGT EINGTERVIVSQLHR SPGVF DSDKGKTHSSGKVLYNAR 175 DIKEQEVYMGEIPLMTDNGTE VINGTERVIVSQLHRSSPGVFHDSDKGKTHSSGKVLYNAR 175 DIKEQE VYMGEIPLMTDNGT NINGTERVIVSQLHR SPGVFEDSDKGKTHSSGKVLYNAR 175 DMQKQI FIGNIPLMNSLGT IVN IY RIVI NQILOSPGIY RSELD---HNGISVYTGT 150 DMQEQT EIGNIPLMNSLGT IVN IY RIVI VQIL OSPGIY RSELD---HNGISVYTST 149

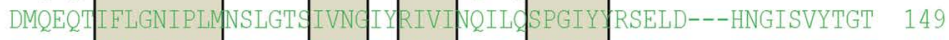

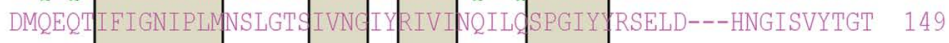

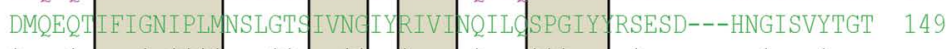

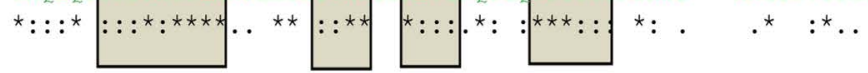


SP|BAEYU9|RPOB PROMH SP |A7FNI3 |RPOB_YERP3 SP I Q1C1U1 |RPOB YERPA SP|A8G8E7 |RPOB - SERP5 sp | C5BHE3 | RPOB EDWI9 SP |A7MQQ9 |RPOB CROS8 Sp |B5XYF5 | RPOB KLEP3 SP |P0A8V2 |RPOB ECOLI sp |Q31U10|RPOB SHIBS sp IQ32AF9|RPOB SHIDS Sp |B5RFK1 |RPOB SALG2 SPIB5BJQ3|RPOB_SALPK sp|P06173|RPOB_SALTY SP | Q9MTM5 | RPOB OENEH SP | Q50A72|RPOB ACOGR SPIQ0G9XO |RPOB DAUCA tr|G1CC85|G1CC 85 SOLTU sp|Q09G54|RPOB PLAOC

sp |B4EYU9|RPOB_PROMH SP |A7FNI3 |RPOB YERP3 SP IQ1C1U1|RPOB_YERPA sp |A8G8E7 | RPOB_SERP5 sp |C5BHE3 |RPOB EDWI9 SP /A7MQQ9 |RPOB CROS8 SP |B5XYF5 |RPOB_KLEP3 SP|POA8V2 |RPOB ECOLT SP $\mid$ Q31U10|RPOB SHIBS sp |Q32AF9|RPOB SHIDS sp | B5RFK1 |RPOB_SALG2 SP|B5BJQ3 |RPOB SALPK SP|P06173|RPOB SALTY SP |Q9MTM5 | RPOB_OENEH SP $\mid$ Q50A72|RPOB_ACOGR SP $\mid$ QOG9XO|RPOB DAUCA tr|G1CC85|G1CC85 SOLTU sp | Q0 9G54 |RPOB_PLAOC

sp |B4EYU9|RPOB_PROMH SP |A7FNI3|RPOB_YERP3 SP |Q1C1U1|RPOB YERPA SP |A8G8E7 | RPOB SERP5 sp |C5BHE3|RPOB_EDWI9 sp |A7MQQ9 |RPOB_CROS8 SP |B5XYF5 | RPOB KLEP3 SP|P0A8V2|RPOB ECOLI SP IQ31U10|RPOB_SHIBS sp IQ32AF9|RPOB_SHIDS sp|B5RFK1 | RPOB SALG2 sp |B5BJQ3|RPOB_SALPK sp |P06173|RPOB_SALTY SP |Q9MTM5 |RPOB OENEH sp|Q50A72|RPOB ACOGR SP|Q0G9XO|RPOB DAUCA tr|G1CC85|G1CC 85 SOLTU

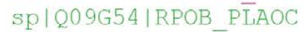

sp |B4EYU9|RPOB_PROMH SP|A7FNI3|RPOB YERP3 Sp IQ1C1U1 |RPOB_YERPA SP |A8G8E7 |RPOB_SERP5 sp | C5BHE3 | RPOB EDWI9 Sp |A7MOO9|RPOB CROS8 sp |B5XYF5 |RPOB_KLEP3 SP | POA8V2 |RPOB_ECOLI sp |Q31U10|RPOB SHIBS SP|Q32AF9|RPOB SHIDS SPIB5RFK1 IRPOB_SALG2 SP|B5BJQ3 |RPOB_SALPK SP|P06173|RPOB SALTY SP |Q9MTM5 |RPOB OENEH SP |Q5QA72|RPOB_ACOGR SP IQOG9XO|RPOB DAUCA

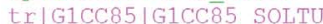
SP IQ09G54 |RPOB PLAOC
IIPYRGSWIDFEFDP DNLFVRIDRRRKLPATIILRAMNYSTEPILNLFFEKTTFEISNN 235 IIPYRGSWIDFEFDP DNLFVRIDRRRKLPATI ILRALNFTTADILDLFFEKVVFEIRDN 235 IIPYRGSWI DFEFDP DNLFVRIDRRRKLPATIILRALNFTTARILDLFFEKVVFEIRDN 235 IIPYRGSWIDEEFDPKDNLFVRIDRRRKLPATI ILRALNYTTEDILDLFFAKVVYEIRDN 235 IIPYRGSWT DFEFDPF DNLFVRIDRRRKLPATIILRALDFTTERILDLFFDKVIFEIRDN 235 IIPYRGSWI DFEFDP DNLFVRIDRRRKLPATIILRALDYTTEDILDLFFEKVVFEIRDN 235 IIPYRGSWIDFEFDPKDNLFVRIDRRRKLPATIILRALNYTTERILDLFFEKVVFEIRDN 235 IIPYRGSWIDFEFDP DNLFVRIDRRRKLPATIILRATNYTTE DILDLFFEKVIFEIRDN 235 IIPYRGSWIDFEFDPHDNLFVRIDRRRKLPATIILRALNYTTERILDLFFEKVIFEIRDN 235 IIPYRGSWIDEEFDP DNLFVRIDRRRKLPATI ILRALNYTTEQILDLFFEKVIFEIRDN 235 IIPYRGSWIDFEFDPKDNLFVRIDRRRKLPATI ILRALNYTTERILDLFFEKVVFEIRDN 235 IIPYRGSWIDFEFDPHDNLFVRIDRRRKLPATIILRALNYTTERILDLFFEKVVFEIRDN 235 IIPYRGSWT.DFEFDPF DNLFVRIDRRRKLPATI ILRALNYTTERILDLFFEKVVFEIRDN 235 IISDWGGR $\mid$ ELEIDR ARIWARVSRKQKISILVLSSAMGSNLREILENVCYPE------- 203 IISDWGGR ELEIDR SRIWARVSRKQKISILVLSSAMGSNLREILDNVCYPE------ 202

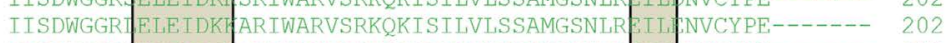
IISDWGGR FETE IDR ARIWARVSRKQKISILVLSSAMGLNLR ETT INVCYPE------ 202 IISDWGGR ELEIDR ARIWARVSRKQKISILVPSSAMGSNLR EILDNVCYPE------- 202

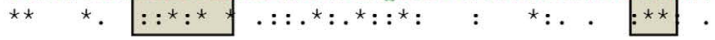

TNDRLSALVETYRMM--RPGEAPTREAAENLFENLFFSEDRYDLSAVGRM KFNRSLGREE 413 TSDRLSALVEIYRMM--RHGE PTREAAENLFENLFFSEDRYDLSAVGRMKENRSLLRDE 413 TSDRLSALVEIYR MM--RAGEA PTREAAENLFENLFFSEDRYDLSAVGRMKENRSLLRDE 413 TSDRLSALVEIYR MM--R AGE PTREAAENLFENLFFSEDRYDLSAVGRMKENR SLLRDE 413 TNDRLSALVEIYRMM--RAGEIPTREAAENLFENLFFSEDRYDLSAVGRMKFNR SLMRDE 413 TTDRLSALVEIYR MM--RAGE PTREAAES LFENLFFSEDRYDLSAVGRMKENRSLLRDE 413 TNDRLSALVEIYR YM--RAGEAPTREAAESLFENLFFSEDRYDLSAVGRM KFNR SLLRDE 413 TNDRLS I VETY YM--RH GE PTREAAES LFENLFFSEDRYDLSAVGRM FNR SLLREE 413 TNDRLSALVEIYRMM--RAGEA PTREAAESLFENLFFSEDRYDLSAVGRMKFNR SLLREE 413 TNDRLSALVEIYR YM--RAGEH PTREAAESLFENLFFSEDRYDLSAVGRM KENRSLLREE 413 TNDRLSALVEIYR YM--RAGEA PTREAAES LFENLFFSEDRYDLSAVGRMKFNR SLLRDE 413 TNDRLSALVETYR MM--RAGEP PTREAAES LFENLFFSEDRYDLSAVGRM KFNRSLLRDE 413 TNDRLSALVEIYR MM--RAGE PTREAAESLFENLFFSEDRYDLSAVGRMKENRSLLRDE 413 --KEN 1 LEFY OESCV GD VESESLCKELHT-KFFQQRCELGRIGRO IMNR RLNLDI 275 ---KEN AILEFY OFACV GD VEFSESCKELOK-KFFQQRCELGRIGRR IMNR RLNLDI 274 ---KEN ATTEFY QFACV GD VFSESICKELQK-KFFQQRCELGKT GRR IMNR RTRLDI 274 ---KEN SILEFY QFACV GD VESESLCKELQK-KFFQQRCELGRIGRR IMNR KLNLDI 274

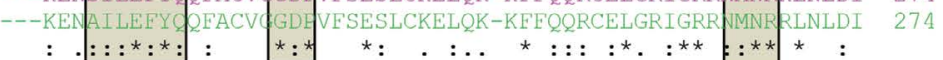

VEGSGILSQEDIIEVMKKLIDIRNGKGEVDD DHL NRRIRSVFEMAENQFRVGLVRVER 473 IEGSGI LSKEDITEVMKKLIDIRNGRGE VDD DHL GNRRIRSV GEMAENQFRVGLVRVE R 473 IEGSGILSKEDITEVMKKLIDIRNGRGE VDD DDHL GNRRIRSVGEMAENQFRVGEVRVER 473 IEGSGILSKDDI IEVMKKLIGIRNGKGE VDD IDHL, GNRRIRSV GEMAENQFRVGLVRVE R 473 IEGSGILSKNDI IEVMKKLIDIRNGKGE VDD HDHL GNRRIRSV JEMAENQFRVGLVRVER 473 IEGSGILSKADI IDVMKKLIDIRNGKGP VDD DHL GNRRIRSV IEMAENQFRVGEVRVER 473 IEGSGILSKDDI IEVMKKLIDIRNGKGE VDD DHL GNRRIRSVGEMAENQFRVGLVRVER 473 TEGSGILSKDDIIDVMKKLIDIRNGKGE VDD DHT NRRTRSV EEMAENQFRVGEVRVF R 473 IEGSGILSKDDI IDVMKKLIDIRNGKGIVDD HDHL GNRRIRSV GEMAENQFRVGLVRVER 473 IEGSGI LSKDDI IDVMKKLIDIRNGKGEVDD DHL GNRRIRSV_FEMAENQFRVGIVRVER 473 IEGSGILSKDDI IDVMKKLIDIRNGKG EVDD IDHL GNRRIRSV SEMAENQFRVGLVRVER 473 IEGSGILSKDDI IDVMKKLIDIRNGKGE VDD DDHLGNRRIRSVGEMAENQFRVGLVRVER 473 IEGSGI LSKDDI IDVMKKLIDIRNGKGE VDD DHL GNRRIRSV IEMAENQFRVGLVRVE $R$ POHNTFLLPRDILAAADHLIGMKFGMGT LD NHI NKRIRSVADLLODOFGLA VVRLE S 335 PQSNTFLLPRDVLAAADHLIGMKFGMGT LDD NHT NKRTRSVADLLQDQFGLA VVRLA N 334 SQNNTFLLPRDILAAADHLIGMKFGMGT EDD NHI NKRIRSVADLIQDQLGLA AVRLEN 334 PQNNTELLPRDILAAADHLIGLKEGMGA DD NHI ANKRIRSVADLLQDQEGLA EVRLAN 334 PQNNTFLLPRDVLAAADHTIGMKFGMGT LDT NHT NKRTRSVADTLQDQEGLA TRLEN 334

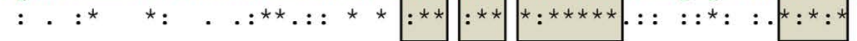

AVKERLSLGDLDA--LMPQDMINAKPISAAVKE EQSSQLSQ MDQWNPLSEITHKRRIS 531 AVKERLSLGDLDT--LMPQDMINAKPISAAVKE F\&SSOLSQ DMDQNNPLSEITHKRRIS 531 AVKERLSLGDLDT--LMPQDMINAKPISAAVKE E F.\$SQ LSQ EMDQNNPLSEITHKRRIS 531 AVKERLSLGDLDT--LMPQDMINAKP ISAAVKE F F SSQ LSQ PMDQNNPLSEITHKRRIS 531 AVKERLSLGDLDM--LMPQDMINAKPISAAVKE E E\$SOLSQ MDQINPLSEITHKRRIS 531 AVKERLSLGDLDT--LMPQDMINAKPISAAVKE FC.SSOLSQ FMDQINNLSEITHKRRIS 531 AVKERLSLGDLDT--LMPQDMINAKPISAAVKE FGSSQ LSQ MDQNNPLSEITHKRRIS 531 AVKERLSLGDLDT--LMPQDMINAKP ISAAVKE E SSQ SQ MDQ NNPL SE ITHK RRIS 531 AVKERLSLGDLDT--LMPQDMINAKPISAAVKE E F\$SSQLSQ MDQNNPLSEITHKRRIS 531 AVKERLSLGDLDT--LMPQDMINAKPISAAVKE E FCSSQ LSQ FMDQNINPLSEITHKRRIS 531 AVKERLSLGDLDT--LMPQDMINAKPISAAVKE F FSSQ LSQ PMDQWNPLSEITHKRRIS 531 AVKERLSLGDLDT--LMPQDMINAKPISAAVKE F\&.\$SQLSQ EMDQINPLSEITHKRRIS 531 AVKERLSLGDLDT--LMPQDMINAKPISAAVKE E FSSOLSQ MDQNNPLSEITHKRRIS 531 VVRRTICGAIRHKL IPTPHNLVTSTPLTTTYES EU HP SO LDRTNPLTOTVHGRKLS 395

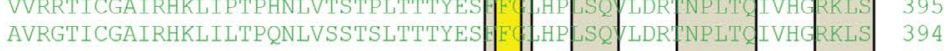
MIRGTIGGALRHKLIPSPQNLVTSTPLTSTYES EO HP SO LDR NPLTOIVHORKLS 394 VVRGTICGATRHKLIPTPQNLVTSPPLTTTYES E EHP SQ LDR NNPLTOIVHGRKLS 394 VVRGTICGAIKHKLIPTPQNLVTSTPLTTTYES Ed HP SO LDR NPLTOMVHG RKLS 394 
SP|B4EYU9|RPOB PROMH SP|A7FNI3 |RPOB YERP3 SP|Q1C1U1|RPOB YERPA SP|A8G8E7|RPOB SERP5 sp|C5BHE3|RPOB_EDWIS sP|A7MQQ9|RPOB_CROS8 sp | B5XYF5 | RPOB KLEP3 SP|POA8V2 |RPOB ECOLI sp |Q31U10|RPOB SHIBS sp|Q32AF9|RPOB_SHIDS sp |B5RFK1 | RPOB SALG2 sp |B5BJQ3 | RPOB SALPK sp |P06173|RPOB_SALTY SP|Q9MTM5 |RPOB OENEH SP 1050 A72 |RPOB ACOGR SPIQOG9XO|RPOB DAUCA tr|G1CC85|G1CC85 SOLTU sp |Q09G54|RPOB_PLAOC

sp|B4EYU9|RPOB PROMH SP IA7FNI3 IRPOB YERP3 SP I Q1C1U1 IRPOB YERPA SP|A8G8E7|RPOB SERP5 Sp |C5BHE3 |RPOB EDWI9 sp |A7MQQ9 | RPOB CROS8 sp I B5XYF5 | RPOB_KLEP3 Sp | POABV2 |RPOB ECOLI sp|Q31U10|RPOB SHIBS sp|Q32AF9 | RPOB_SHIDS sp | B5RFK1 |RPOB SALG2 Sp |B5BJQ3 |RPOB SALPK sp|P06173|RPOB_SALTY SP IQ9MTM5 |RPOB_OENEH sp |050A72 |RPOB ACOGR SPIQ0G9X0|RPOB DAUCA tr|G1CC85|G1CC $\overline{8} 5$ SOLTU sp|Q09G54|RPOB_PLAOC

sp | B4EYU9|RPOB_PROMH sp|A7FNI3|RPOB YERP3 SP $\mid$ Q1C1U1 |RPOB YERPA sp|A8G8E7|RPOB_SERP5 SP |C5BHE3|RPOB_EDWI9 sp/A7MOQ9/RPOB CROS8 sp|B5XYF5 |RPOB KLEP3 sp | POA 8V2 | RPOB ECOLI sp |Q31U10|RPOB_SHIBS sp |032AF9 |RPOB SHIDS sp |B5REK1 |RPOB_SALG2 sp|B5BJQ3 IRPOB_SALPK sp|P06173|RPOB_SALTY SP IO9MTM5 IRPOB OENEE sp | Q5QA72|RPOB_ACOGK Sp |Q0G9X0 |RPOB_DAUCA tr|G1CC85|G1CC85 SOLTU sp|Q09G54|RPOB PLAOC

sp|B4EYU9|RPOB PROMH sp |A7FNI3 |RPOB YERP3 sp IQ1C1U1|RPOB_YERPA sp|A8G8E7|RPOB SERP5 SP / C5BHE3 IRPOB EDWI9 sp |A7MQQ9 |RPOB_CROS8 SP|B5XYF5 | RPOB KLEP3 SP I POA8V2 |RPOB ECOLI sp | Q31U10 |RPOB SHIBS sp|Q32AF9|RPOB_SHIDS sp |B5RFK1 | RPOB SALG2 sp/B5BJQ3 |RPOB SALPK sp | P06173|RPOB_SALTY SP I Q9MTM5 | RPOB OENEE sp $1050 A 72 \mid$ RPOB ACOGE SPIQOG9XO IRPOB DAUCA tr|G1CC85|G1CC $\overline{8} 5$ SOLTU splQ09G54|RPOB PLAOC
AI GPGGLT RAGEEY VVHPTHYGRVCPIE PEGNIGL NSLSVYAQTNEYG LETPY 591 AI GPGGLTAERAGFEYR DVHPTHYG VVCPIE PEGPNIGL NSLSVYAQTNEYG LETPY 591 AI GPGGLTHERAGFEYRDVHPTHYYGVCPIE PPEGPNIGLENSLSVYAQTNEYG LETPY 591 AI GPGGLTHERAGFEYR DVHPTHYGAVCPIEFPEGPNIGL_NSLSVYAQTNEYG LETPY 591 AI GPGGLT E RAGEEYR DVHPTH YGA VCPIE PPEPNIGL NSLSVYAQTNEYG LETPY 591 AI GPGGLTEERAGFEYR DVHPTH YGRVCPIE PPEGPNIGL NSLSVYAQTNEYG LETPY 591 AI GPGGLTAERAGFEVR DVHPTH YG RVCPIE PPERNIGL NSLSVYAQTNEYG LETPY 591 AIGPGGLT ERGEEY DVHPTH GLVPIE PEGPNIGLENSLSVYAQTNEYG LETPY 591 AI GPGGLTH ERAGFEYR DVHPTH YGARCPIE PEGPNIGL NSLSVYAQTNEYG LETPY 591 AI GPGGLTHERAGFEYR DVHPTH YGEVCPIE PPEGPNIGLENSLSVYAQTNEYG LETPY 591 AI GPGGLTHERAGFEVRDVHPTHYGRVCPIEFPEGPNIGL_NSLSVYAQTNEYG ELTPY 591 AI GPGGLTHERAGFEYR PVHPTH YGEVCPIE P E EPNIGL NSLSVYAQTNEYG LETPY 591 AI GPGGLTHERAGEEYR PVHPTH YGRVCPIE PPEGPNIGL NSLSVYAQTNEYG LETPY 591

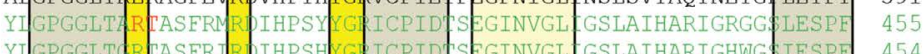

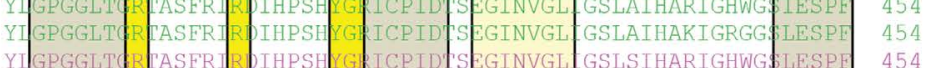

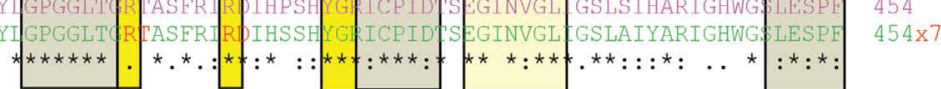

QYMDVSTQQVVSVGASLIPELEHDDANRALAGANMQRQAVP PLRGDKPLVGTGMERAVAV 710 DYMDVSTOOIVSVGASLIPELEHDDANRAIMG NNMOROAVP PLRADKPLVGTGMERAVAV 710 DYMDVSTQQIVSVGASLIPELEHDDANRALMG ANMQRQAVP ILRADKPLVGTGMERAVAV 710 DYMDVSTQQVVSVGASLIPELEHDANRALMGANMQRQAVP PLRADKPLVGTGMERAVAV 710 DYMDVSTQQVVSVGASLIPELEHDDANRALMGANMQRQAVP PLRADKPLVGTGMERAVAV 710 DYMDVSTQQVVSVGASLIPELEHDDANRALIIGANMQRQAVP PLRADKPLVGTGMERAVAV 710 DYMDVSTQQVVISVGASLIP LEHDDANRAT. IG ANMQRQAVP PLRADKPLVGTGMERAVAV 710 DYMDVSTOOVVSVGASLIP DDANRAT, GG NMOROAVP ELRADKPLVGTGMERAVAV 710 DYMDVSTQQVVSVGASLIPELEHDDANRALMGANMORQAVP PLRADKPLVGTGMERAVAV 710 DYMDVSTQQVVSSGASLIPELEHDDANRALAG ANMQRQAVP PLRADKPLVGTGMERAVAV 710 DYMDVSTQQVVSSGASLIP LEHDDANRALIIGANMQRQAVP FLRADKPLVGTGMERAVAV 710 DYMDVSTOOVVSVGASLIPELEHDANRAL,VGANMOROAVP PLRADKPLVGTGMERAVAV 710 DYMDVSTQQVVSVGASLIP LEHDDANRALIIGANMQRQAVP FLRADKPLVGTGMERAVAV 710

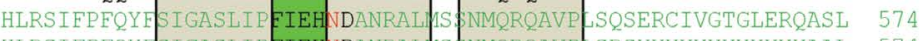
HLRS IFPFQYE SIGASLIP IEH NDANRA IS N NMORQAVP SRSXXXXXXXXXXXXAAL 574

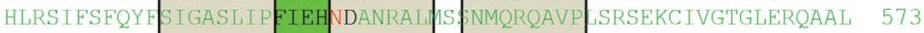
HLRSIFPFQY SIGASLIP NDANRATIS NNMQRQAVP SRSEKCIVGTGLERQAAL 573 HFRS IFPFQY ESIGASLIP EIEH NDANRATIS NNMQRQAVP SQSEKCIVGTGLERQAAL 573

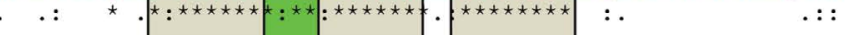

SEFTAVAKRGGTVQYVDASRIVIKVNEDETYAGEAGIDIYSLTKY RSNQNTCINQTPC 770 VTSVAKRGGTVQYVDASRIVIKVNEDEMHPGEAGIDIYNLTKYTRSNQNTCINQMPC 770 VTSVAKRGGTVOYVDASRIVIKVNEDEMHPGEAGIDIYNLTKYTRSNQNTCINQMPC 770 VTAVARRGGVIQYVDASRIVIKVNEDEMLAGEAGIDIYNLTKY RSNQNTC INQMPC 770 VTSVAKRGGMVQYVDASRIVIKVNEDEMFPGEAGIDIYNLTKYDRSNQNTCISQMPC 770 SGTAVAKRGGIVOYVDASRIVIKVNEDEMYPGEAGIDIYNLTKYTRSNONTCINOMPC 770 GVTAVAKRGGTVQYVDASRIVIKVNEDEMYPGEAGIDIYNLTKY'RSNONTCINQMPC 770 TTAVAKRGGVVQYVDASRIVIKVNEDEMYPGEAGIDIYNLTKY RSNONTCENQMPC 770 NTAVAKRGGVVQYVDASRIVIKVNEDEMYPGEAGIDIYNLTKYTRSNQNTCINQMPC 770 GTAVAKRGGVVOYVDASRIVIKVNEDEMYPGEAGIDIYNLTKY'RSNONTCINOMPC 770 GTAVAKRGGTVQYVDASRIVIKVNEDEMYPGEAGIDIYNLTKYTRSNQNTC INQMPC 770 FTAVAKRGGTVQYVDASRIVIKVNEDEMYPGEAGIDIYNLTKYTRSNQNTCINQMPC 770 SEVTAVAKRGGTVQYVDASRIVIKVNEDEMYPGEAGIDIYNLTKYTRSNQNTC INOMPC 770 -TAIAEREGKIIYTNSDKIILSGSG-------DTLSIPLVMY RSNKNTW MHOKPQ 626 - JSAIAECEGKIIYTDTHKIVLSGHG-_-_--DTISIPLVMY RSNKNTCMHQNPQ 626 FLAIAEHEGKVIYTDTDKILLSGNG--------DTLNIPLVMY RSNKNTC MHQKPQ 625 ALAIAEREGRIVYTNTDKILLAGNG-------DILSIPLVIY RSNKNTCMHOKLR 625 IST TAEHEGKI IYTDTDKITLLGNG-------DTLSIPLVMY RSNKNTCMYQKPQ 625

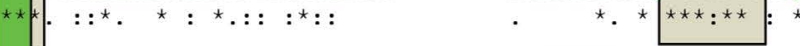

VSLGE VERGDVLADGPSTDI GELALGQNMR VAFMPWN \&YNYELSILVSERVVDEDRFTT 830 VNLGE IERGDVLA DGPSTDI GELALGQNMR FAFMPWN \&YNFEL SILVSERVVEEDRFTT 830 VNLGEH IERGDVLADGPSTDI GELALGQNMR VAFMPWN $\$$ YNFELSILVSERVV EEDRFTT 830 VNLGE IIERGDVLADGPSTDIGELALGQNMRVAFMPWN \$YNFELSILVSERVVDEDRFTT 830 VSLGE HIERGDVLADGPSTDI GELALGQNMR VAFMPWN \&YNFELSILVSERVV REDRFTS 830 VSLNEHVERGDVLADGPSTDI GELALGQNMR VAFMPWN\&YNFEDSILVSERVVEEDRFTT 830 VSLGEH IERGDVLADGPSTDI GELALGQNMR FAFMPWN\&YNFELSILVSERVVREDRFTT 830 VSLGEHVERGDVLA DGPSTDI GELALGQNMR AEMPWN $\quad$ SILVSERVVDEDRFTT 830

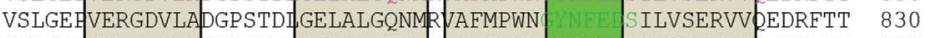
VSLGE VVERGDVA DGPSTDI GELALGQNMR VAFMPWN\&YNFED SILVSERVVEEDRFTT 830 VSLGE HVERGDVLADGPSTDI GELALGQNMRVAFMPWN FYNFELSILVSERVVDEDRFTT 830 VSLGE HVERGDVLADGPSTDI GELALGQNMR VAFMPWN \&YNFELSILVSERVVREDRFTT 830 VSLGEHVERGDVLA DGPSTDI GELALGQNMR FAFMPWN \&YNFEDSILVSERVVEEDRFTT 830 VPRGK IKKGQILA GAATV GEILALGKNV LAYMPWE VRRGK IKKGQILAPGAATV GELALGKNVL AYMPWE N AVLISERLVEDIYTS 686

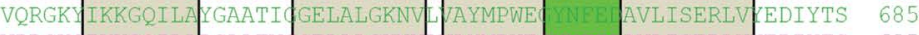
VPRGK IKKGQILA PGAATV GELALGKNVL AYMPWE $\quad$ AVLISERLVEDIYTS 685 VRRGKA IKKGQIVA DGAATV GETALGKNV AYMPWE AVLISERTV GDIYTS 685

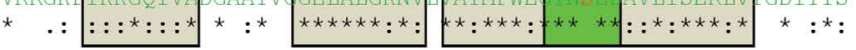

10
10
0
0
0
0
0
0
0
0
0
4
4
3
3
3

.

$x 7$


sp|B4EYU9|RPOB PROMH SD|A7FNT3 IRPOB YERP3 SP IQ1C1U1 IRPOB YERPA SP|A8G8E7 |RPOB SERP5 SP|C5BHE3|RPOB EDWI9 sp |A7MQQ9|RPOB CROS8 SP IB5XYE5 |RPOB KLEP3 SP |POA8V2 |RPOB ECOLI SP $\mid 031$ U10|RPOB SHIBS sp|Q32AF9|RPOB_SHIDS SP IB5REK1 |RPOB_SALG2 sp|B5BJQ3|RPOB SALPK SP|P06173|RPOB SALTY SP | Q9MTM5 | RPOB OENEH SPIQ5QA72 |RPOB - ACOGR SPIQ0G9XO|RPOB DAUCA tr|G1CC85|G1CC8 5 SOLTU sp|Q09G54|RPOB_PEAOC

SP|B4EYU9|RPOB PROMH SP |A7FNI3 |RPOB YERP3 Sp|Q1C1U1 |RPOB YERPA SP|A8G8E7 |RPOB - SERP5 Sp | C5BHE3 |RPOB EDWI9 SP |A7MQQ9 |RPOB CROS8 Sp|B5XYF5 |RPOB KLEP3 SP | POA8V2 |RPOB ECOLI SPIQ31U10|RPOB_SHIBS sp IQ32AF9|RPOB SHIDS sp|B5RFK1|RPOB SALG2 SP|B5BJQ3।RPOB_SALPK sp|P06173|RPOB_SALTY SD|O9MTM5 IRPOB OFNEH SP |Q50A72 |RPOB ACOGR Sp |QOG9XO|RPOB_DAUCA tr|G1CC85|G1CC $\overline{8} 5$ SOLTU sp|Q09G54|RPOB_PËAOC

SP|B4EYU9|RPOB PROMH SP|A7FNI3 |RPOB YERP3 SPIQ1C1U1 |RPOB YERPA SP|A8G8E7 |RPOB_SERP5 Sp|C5BHE3|RPOB EDWI9 sp |A7MQQ9|RPOB_CROS8 SP|B5XYF5 |RPOB_KLEP3 SP|P0A8V2 |RPOB-ECOLI SPIQ31U10|RPOB SHIBS SPIQ32AF9|RPOB_SHIDS SP|B5RFK1 IRPOB SALG2 Sp|B5BJQ3|RPOB SALPK SP|P06173|RPOB SALTY SP | Q9MTM5 | RPOB OENEH SP|Q5QA72 |RPOB ACOGR sp |QOG9XO|RPOB DAUCA tr|G1CC85|G1CC $\overline{8} 5$ SOLTU sp |Q09G54 |RPOB_PLAOC

SP|B4EYU9|RPOB_PROMH SP|A7FNI3 |RPOB_YERP3 SP|O1C1U1 IRPOB YERPA SP |A8G8E7 |RPOB SERP5 SPIC5BHE3 IRPOB_EDWI9 SP|A7MQQ9 |RPOB CROS8 SP |B5XYF5 | RPOB KLEP3 Sp | P0A8V2|RPOB_ECOLI spIQ31U10|RPOB_SHIBS SP|Q32AF9|RPOB_SHIDS sp|B5RFK1 |RPOB SALG2 SP|B5BJQ3|RPOB SALPK SP|P06173|RPOB_SALTY SP|Q9MTM5 |RPOB OENEH SP|Q50A72 |RPOB ACOGR SP|QOG9XOIRPOB DAUCA tr|G1CC85|G1CC 85 SOLTU SPIQ09G54|RPOB PLAOC
IHIQELACVSRDTKLGPEEITADIPNVGEAALSKLDESGIVIGAEVKG DILVGKVTP IHIOELACVSRDTKL GPE EITADI PNVGEAALSKLDESGIV I GAEVTG GDILVGKVTP IHIQELACVSRDTKLFPEEITADIPNVGEAALSKLDESGIV IGAEVTG \$DILVGKVTP IHIQELACVSRDTKLFPEEITADIPNVGEAALSKLDESGIV IGAEVTG \$DILVGKVTP IHIOELACVSRDTKL FPEEITADIPNVGEAALSKLDESGIV IGAEVKG DDILVGKVTPK IHIOELACVSRDTKLGPEEITADIPNVGEAALSKLDESGIV¥IGAEVTG\&DILVGKVTP IHIQELACVSRDTKLGPEEITADIPNVGEAALSKLDESGIV IGAEVTG\$DILVGKVTPI IHIQELACVSRDTKL FPEEITADIPNVGEAALSKLDESGIV IGAEVTG DDILVGKVTP IHIOELACVSRDTKLGPEEITADIPNVGEAALSKLDESGIVҰIGAEVTG\&DILVGKVTP IHIQELACVSRDTKLGPEEITADI PNVGEAALSKLDESGIVYIGAEVTGGDILVGKVTP IHIQELACVSRDTKL GPEEITADI PNVGEAALSKLDESGIV I GAEVTG \$DILVGKVTP IHIQELACVSRDTKLGPEEITADIPNVGEAALSKLDESGIV IGAEVTG\$DILVGKVTP THTOELACVSRDTKL GPE EITADI PNVGEAALSKLDESGIVY TGAEVTG \$DILVGKVTP FHIRKYEMQTHVTSQ SPE RITNEIPHLEAHLLRNLDKN GIVYMGSWVEA EHIRKYEIQTHVTSQ FHIRKYETOTHVTSO FHIRKYEIHTHVTSO FHIRKYETOTHUTSO

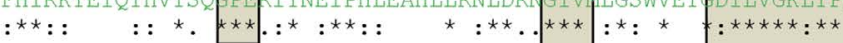
G---ETQL TPEEKLLRAIFG KASDVKDSS LRVPNGVSGTVIDVQVFTRDGVEKDKRALE G---ETQL PPEEKLLRAI FG K KASDVKDSS LRVPNGVSGTVIDVOVFTRDGVEKDKRALE G---ETQL TPEEKLLRAI FGH KASDVKDSSLRVPN GVSGTVIDVUVFTRDGVEKDKRALE G---ETOL PPEEKLLRAI FGH KASDVKDSSLRVPN GVSGT IDVOVFTRDGVEKDKRALE G---ETQL TPEEKLLRAT FGH KASDVKDSS LRVPN SVSGT IIDVQVFTRDGVEKDKRALE G---ETQL'PEEKLLRAIFGHKASDVKDSSLRVPNGVSGT VIDVQVFTRDGVEKDKRALE G---ETOL'PPEEKLLRAI FG H KASDVKDSSLRVPN GVSGTVIDVOVFTRDGVEKDKRALE G---ETQL PEEKLLRAI G KASDVKDSS LRVP GVSGT IDVQVETRDGVEKDKRALE G---ETQL TPEEKLLRAI FGH KASDVKDSS LRVPN GVSGTVIDVQVFTRDGVEKDKRALE G---ETQL PPEEKLLRAI FG H KASDVKDSSLRVPN GVSGT I DVQVFTRDGVEKDKRALE G---ETOL TPEEKLLRAIFGH KASDVKDSS LRVPN GVSGTVIDVOVFTRDGVEKDKRALE G---ETQL'PEEKLLRAIEG K KASDVKDSS LRVPN GVSGT I DVQVFTRDGVEKDKRALE G---ETQL PDEEKLLRAIFGH KASDVKDSSERVPN GVSGTVIDVQVFTRDGVEKDKRALE MAEEESSY PEERLLRAI G QISTSKETC KLP GGRGR IDVRWIOKRGG------TAN-ESSY PEDRLLRAI G OVSTAKETC LKLP GGRGR IDVRWIOKKGG------MVK-ESSY PEDRLLRAT G EVSTSKETC KLP GGRGR IDV RWIQKRVG------VVK-ESSY PEDRLILRAT MAK- FSSY DFDRTTRAT

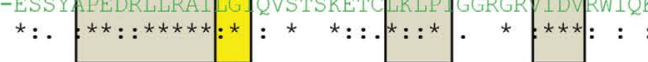

ONOLEOLAEOYDELKAEFAKKLEAKRRKITOGDDLAPGVLKIVKVYLAVKROIO HGDKMA ONQLEOLAEOYDEMKSEFEKKMDAKRRKITOGDDLAPGVLKIVKVYLAVKRQIOH GDKMA QNQLEQLAEQYDEMKSEFEKKMDAKRRKITQGDDLAPGVLKIVKVYLAVKRQIQHGDKMA QNQLEQLAEQYDELKSDFEKKLDAKRRKITQGDDLAPGVLKIVKVYLAVKRQIQH GDKMA QNQLEQLAEQYDELKAEFEKKLEAKRRKITQGDDLAPGVLKIVKVYLAVKRQIQ IGDKMA QNQLEQLAEQYDELKHEFEKKLEAKRRKITQGDDLAPGVLKIVKVYLAVKRQIQHGDKMA QNQLEQLAEQYDELKHEFEKKLEAKRRKITQGDDLAPGVLKIVKVYLAVKRRIQ GDKMA ONOLEOLAEOYDELKHEFEKKLEAKRRK TTOGDDLAPGVLKTVKVYLAVKRRTO GDKMA ONOLEOLAEOYDELKHEFEKKLEAKRRKITOGDDLAPGVLKIVKVYLAVKRRIOH GDKMA QNQLEQLAEQYDELKHEFEKKLEAKRRKITQGDDLAPGVLKIVKVYLAVKRRIQ GDKMA QNQLEQLAEQYDELKHEFEKKLEAKRRKITKGDDLAPGVLKIVKVYLAVKRRIQ HGDKMA QNQLEQLAEQYDELKHEFEKKLEAKRRKITQGDDLAPGVLKIVKVYLAVKRRIQHGDKMA QNQLEQLAEQYDELKHEFEKKLEAKRRKITQGDDLAPGVLKIVKVYLAVKRRIQ GDKMA -

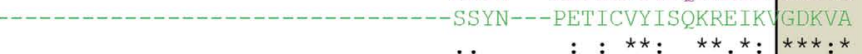
$:: * *: * * . *: * * * *$

GRHGNKGVISKIN PI EDMPY $\$$ ENGNP VDLVLN H VPSRMNIGQILE EHLGMAAKGIGDK 1127 GRHGNKGVISKIN P IEDMPY $\$$ ENGTP VDIVLN L LVPSRMNIGQILE HLGMAAKGIGEK 1127 GRHGNKGVISKINPI EDMPY EENGTP VDIVLN H L EVPRMNIGQILE VHLGMAAKGIGEK 1127 GRHGNKGVISKIN PIEDMPY NENGTP VDIVLN A L VPSRMNIGQILE WHLGMAAKGIGEK 1127 GRHGNKGVISKIN P I EDMPY ENGTP VDIVLN L LVPSRMNIGQILE HLGMAAKGIGDK 1127 GRHGNKGVISKIN PIEDMPY DENGTP VDIVLN AL EVPSRMNIGQILE THLGMAAKGIGDK 1127 GRHGNKGVISKINPI EDMPH 4 ANGTPVDIVLN HL FVPSRMNIGQILENHLGMAAKGIGDK 1127 GRHGNKGVISKIN P I EDMPY ENGTH VDIVLN L VPSRMNIGQILE HLGMAAKGIGDK 1127 GRHGNKGVISKIN PI EDMPY ENGTPVDIVLN PL FVPSRMNIGQI LE FHLGMAAKGIGDK 1127 GRHGNKGVISKIN P IEDMPY QENGT PVDIVLN L EVPSRMNIGQILE WHLGMAAKGIGDK 1127 GRHGNKGVISKINPIEDMPY DENGTPVDIVLN PLEVPSRMNIGQILE HLGMAAKGIGDK 1127 GRHGNKGVISKIN PI EDMPY ENGTE VDIVLN L LVPSRMNIGQILE THLGMAAKGIGDK 1127 GRHGNKGVISKIN P I EDMPY ENGTP VDIVLNH L VVPSRMN IGQI LE PHLGMAAKGIGDK 1127

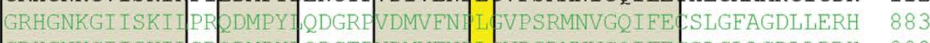
GRHGNKGT TSKT T SR DDMPY QDGTH DDMVFNT T V VPSRMNVGQT FE ESLGTAGDLTDDRH 88 ?

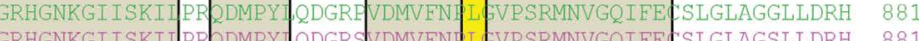
GRHGNKGIISKIT PR PDMPY QDGRS VDMVENI L VPSRMNVGQIFE ESLGLAGSLLDRH 881

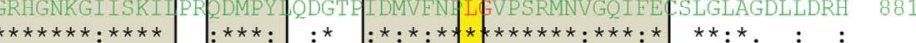

890 890 890 890 890 890 890
890 890 890
746 746
745 45 5 47 47 47 947 (9) 47 47 947
798 96 96 
sp |B4EYU9 |RPOB_PROMH SP |A7FNI3 |RPOB YERP3 sp I Q1C1U1 |RPOB_YERPA SP|A8G8E7|RPOB SERP5 sp |C5BHE3 |RPOB_EDWI9 sp |A7MQQ9 |RPOB CROS8 SP | B5XYF5 |RPOB_KLEP3 Sp | P0A8V2 |RPOB ECOLI SP $\mid Q 31$ U10|RPOB_SHIBS sp | Q32AF9 | RPOB_SHIDS sp |B5RFK1|RPOB_SALG2 sp |B5BJQ3 |RPOB_SALPK sp | P06173|RPOB SALTY Sp | Q9MTM5 | RPOB_OENEH sp | Q50A72 | RPOB ACOGR SP | QOG9XO |RPOB DAUCA

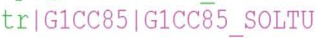
sp | Q09G54 |RPOB_PLAOC

SP |B4EYU9|RPOB_PROMH SP|A7FNI3|RPOB YERP3 SP |Q1C1U1|RPOB_YERPA Sp |A8G8E7|RPOB_SERP5 sp |C5BHE3|RPOB_EDWI9 sp |A7MQQ9 | RPOB CROS8 SP|B5XYF5 |RPOB KLEP3 Sp | POA 8V2 |RPOB ECOLI sp | Q31U10|RPOB_SHIBS sp |Q32AF9|RPOB_SHIDS Sp |B5RFK1 |RPOB_SALG2 sp |B5BJQ3|RPOB_SALPK sp|P06173|RPOB SALTY SP | Q9MTM5 | RPOB_OENEH SP $\mid 050$ A $72 \mid$ RPOB ACOGR SP $\mid$ QOG9XO|RPOB DAUCA tr|G1CC85|G1CC85 SOLTU Sp | Q09G54 |RPOB_PËAOC

sp |B4EYU9 |RPOB_PROMH SP $\mid$ A7FNI3 |RPOB YERP3 Sp IQ1C1U1|RPOB_YERPA Sp|A8G8E7|RPOB SERP5 sp |C5BHE3|RPOB_EDWI9 sp |A7MQQ9 |RPOB CROS8 SP |B5XYF5 |RPOB KLEP3 Sp |POA8V2 |RPOB ECOLI sp |Q31U10|RPOB_SHIBS sp | Q32AF9 |RPOB_SHIDS sp |B5RFK1 |RPOB SALG2 sp |B5BJQ3|RPOB_SALPK sp |P06173|RPOB_SALTY

SP| Q9MTM5|RPOB OENEH SP|Q5QA72|RPOB_ACOGR Sp| QOG9XO|RPOB DAUCA tr|G1CC85|G1CCי55 SOLTU sp | Q09G54|RPOB_PLAOC
DGAEEMEIKEMLKLADLPTSGQITIFDGRTGQ QFERPVTVGYMYMLKLNHLVDDKMHARS 1247 DGATEKEIKELLLLGGLPTSGQITIFDGRTGPQFERQVTVGYMYMLKLNHLVDDKMHARS. 1247 DGATEKEIKELLQLGGLPTSGQITZ FDGRTG耳QFERQVTVGYMYMLKLNHLVDDKMHARS 1247 DGAKESEIKQLLEMGGIPTSGQITIFDGRTGEQFERQVTVGYMYMLKLNHLVDDKMHARS 1247 DGAKEREIKGLLELGGIPTSGQITI YDGRTGERFERQVTVGYMYMLKLNHL_VDDKMHARS 1247 DGAKEAEIKELLQLGDLPTSGQITIFDGRTGRQFERPVTVGYMYMLKLNHLVDDKMHARS 1247 DGAKEAEIKELLQLGDLPTSGQITI FDGRTGHQFERPVTVGYMYMLKLNHLVDDKMHARS 1247 DGAKEAEIKELLKLGDLPTSGQIR YDGRTG QEERPVTVGYMYMLKLNHI IDDKMHARS 1247 DGAKEAEIKELLKLGDLPTSGQIRIYDGRTGHQFERPVTVGYMYMLKLNHLVDDKMHARS 1247 DGAKEAEIKELLKLGDLPTSGQIRLYDGRTGLQFERPVTVGYMYMLKLNHLJVDKMHARS 1247 DGAKEAEIKELLKLGDLPTSGQITIFDGRTGHQFERPVTVGYMYMLKLNHLVDDKMHARS 1247 DGAKEAEIKELLKLGDLPTSGQITI FDGRTGEQFERPVTVGYMYMLKLNHLJVDKMHARS 1247 DGAKEAEIKELLKLGDLPTSGQITIFDGRTGEQFERPVTVGYMYMLKLNHLVDDKMHARS 1247 DG-------WVEEPEYPGKSR EDGRTG PEEQPVIIGKPYILKLIHONDDKIHORS 964 ----------WVEEPEYPGKSR EDGRTG PEEQPVLIGKSYILKLIHQ DDDKIHCRS 963 ----------WVEEPEYPGKSR EDGRTG PEEQPVIIGKPYILKLIHQ NDDKIHCRS 962

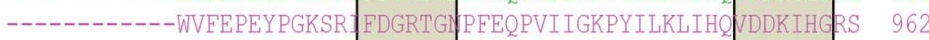
--------WVEEEYPGKSR EDGRTG PEEQPVLIGKSYILKLIHO DDDKLCRS 962

TGSYSIVTQQPLGGKAO FGGQR FEMEVWALEA GAAYTLEEMLT KSDPVNGRTKMYKN 1307 TGSYSLVTQQPLQGKADFGGQR E EMEVWALEA GAAAYLQEMLT V KDDVVNGRTKMYKN 1307 TGSYSLVTQQPLGGKAdFGGQR E EMEVWALEA GAAYTLQEMLT WSDDVNGRTKMYKN 1307 TGSYSIVTQQPLGGKAdFGGQR E EMEVWALEA GAAYTLQEMLT KSDDVNGRTKMYKN 1307 TGSYSLVTQQPLCGKADFGGQR FEEMEVWALEAYGAAYTLQEMLT VKSDDVNGRTKMYKN 1307 TGSYSLVTQQPLCGKADFGGQR E EEMEVWALEA Y GAYTLQEMLT WKSDDVNGRTKMYKN 1307 TGSYSIVTQQPLQGKAdFGGQRF EEMEVWALEA YAAYTLQEMLT KSDDVNGRTKMYKN 1307

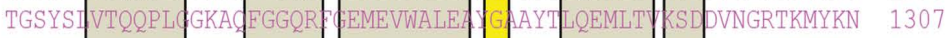
TGSYSLVTQQPL GGKAdFGGQR E EEMEVWALEA GAAYTLQEMLT KSDDVNGRTKMYKN 1307 TGSYSIVTQQPLGGKADFGGQR F EEMEVWALEA GAAYTLQEMLT KSDDVNGRTKMYKN 1307 TGSYSIVTQQPLAGKAdFGGQRF EEMEVWALEA YAAYTLQEMLT KSDDVNGRTKMYKN 1307 TGSYSLVTQQPLQGKAOFGGQR F EEMEVWALEA GAAYTLQEMLT KSDDVNGRTKMYKN 1307 TGSYSIVTQQPLAGKADFGGQR FEMEVWALEA GAAYTLQEMLT KSDDVNGRTKMYKN 1307

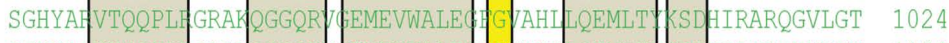

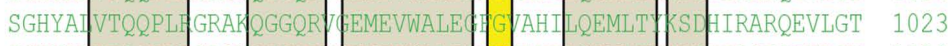

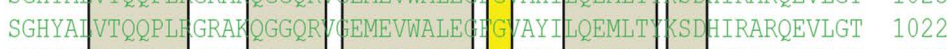

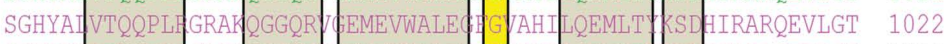
SGHYA VTQQPL GRAI GGGQR J EMEVWALE

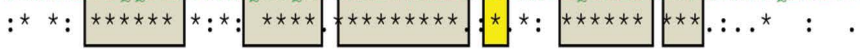

I-VDGNHQMEPGM PESFNVLLKEIRSLGINIELEDE--------- 1342

I-VDGDHRMEPGMPESFNVLLKEIRSLGINIELEEE--------- 1342

I-VDGDHRMEPGMPESFNVLLKEIRSLGINIELEEE---------- 1342

I-VDGDHRMEPGM PESFNVLLKEIRSLGINIELEGE---------- 1342

I-VDGSHQMEPGMPESFNVLLKEIRSLGINIELEDE---------- 1342

I-VDGNHQMEPGMPESFNVLLKEIRSLGINIELEDE----------- 1342

I-VDGNHQMEPGM PESFNVLLKEIRSLGINIELEDE---------- 1342

I-VDGNHQMEPGM PESFNVLILEIRSLGTNIETEDE---------- 1342

I-VDGNHQMEPGMPESFNVLLKEIRSLGINIELEDE---------- 1342

I-VDGNHQMEPGMPESFNVLLKEIRSLGINIELEDE---------- 1342

I-VDGNHQMEPGMPESFNVLLKEIRSLGINIELEDE----------- 1342

I-VDGNHQMEPGMPESFNVLLKEIRSLGINIELEDE----------- 1342

I-VDGNHQMEPGM PESFNVLLKEIRSLGINIELEDE----------- 1342

TILGGTIPKPED PESFRLLVRELRSTALELNHFLVSEKNFQTNRKEA 1072

TIIGGTIPTPEDA PESFRLIVRELRSLALELNHFLVSEKNEQINRKEA 1071

TIIGGAIPNPEDA PESERLLVRELRSLALELNHFFVSEKTEKIKRKEA 1070

TIIGGTIPNPEDAPESFRLLVRELRSLALELNHELVSEKNFQINRKEA 1070

TIVGGTIPSPEDA PESFRLLVRELRSLALELNHELVSEKNEQINRKEA 1070

$\therefore *^{*} \quad .{ }^{* * \star} .:^{*}::^{\star}:{ }^{* \star} .::::$

Figure 5. MSA of the $\beta$ subunits of eubacteria and chloroplast MSU RNAPs. 


\begin{tabular}{|c|}
\hline sp|B4EYU9|RPOB_PROMH Proteus mirabilis \\
\hline sp|A7FNI3|RPOB_YERP3 Yersinia pseudotuberculosis \\
\hline sp|Q1C1U1|RPOB_YERPA Yersinia pestis \\
\hline sp|A8G8E7|RPOB_SERP5 Serratia proteamaculans \\
\hline sp|C5BHE3|RPOB_EDWI9 Edwardsiellaictaluri \\
\hline sp|A7MQQ9|RPOB_CROS8 Cronobacter sakazakii \\
\hline sp|B5XYF5|RPOB_KLEP3 Klebsiella pneumonia \\
\hline sp|P0A8V2|RPOB_ECOLI Escherichia coli K12 \\
\hline sp|Q31U10|RPOB_SHIBS Shigella boydii \\
\hline sp|Q32AF9|RPOB_SHIDS Shigella dysenteriae \\
\hline sp|B5RFK1|RPOB_SALG2 Salmonella gallinarum \\
\hline sp|B5BJQ3|RPOB_SALPK Salmonella paratyphi A \\
\hline sp|P06173|RPOB_SALTY Salmonella typhimurium \\
\hline $\begin{array}{l}\text { sp|Q9MTM5|RPOB_OENEH Oenothera elata subsp. hookeri } \\
\text { (herbaceous flowering plants) }\end{array}$ \\
\hline $\begin{array}{l}\text { sp|Q5QA72|RPOB_ACOGR Acorus gramineus } \\
\text { (monocot flowering plants) }\end{array}$ \\
\hline sp|Q0G9X0|RPOB_DAUCA Daucus carota (wild carrot) \\
\hline $\operatorname{tr|G1CC85|G1CC85\_ SOLTU~Solanum~tuberosum~}$ \\
\hline $\begin{array}{c}\mathrm{sp}|\mathrm{Q} 09 \mathrm{G} 54| \mathrm{RPOB} \text { PLAOC Platanus occidentalis } \\
\text { (American planetree) }\end{array}$ \\
\hline
\end{tabular}

Figure 5 shows the 'mix and match' MSA analysis of $\beta$ subunits of eubacterial and chloroplast MSU RNAPs. There are large numbers of conserved motifs and some are found to be long stretches (highlighted) and a small number of diads and triads among them. The catalytic, template and substrate binding motifs are highlighted in yellow. The possible metal binding regions are highlighted in green. The E. coli and Solanum tuberosum (potato) $\beta$ subunits are used as the standard for numbering and highlighted in magenta. It is interesting to note that the 'gatekeeper pair' YG and its catalytic $\mathrm{R}$ are strictly conserved, including distance conservation, in all the eubacterial and chloroplast $\beta$ subunits of the MSU RNAPs and matches exactly. However, only one YG is aligned in the $\beta$ subunits of both these MSU RNAPs at the expected catalytic region. Additionally, one invariant FG and an LG pair are seen in both the $\beta$ subunits making the total template binding pairs as 3 as discussed elsewhere. In both the cases, the catalytic $\mathrm{R}$ is placed at $7^{\text {th }}$ position downstream from the YG pair which suggests again that the initiation subunit in both the cases are similar in structure and hence likely in function. As discussed earlier, the $\beta$ subunit makes many aborted transcripts up to $7 \mathrm{nts}$ and once it crosses $7 \mathrm{nts}$, the initiation transcript fits into the next elongation subunit's active centre, i.e., the $\beta^{\prime \prime}$ subunit and goes for elongation ${ }^{22}$. This is further confirmed by quantitation of the preinitiation complex by high-resolution gel electrophoresis technique which showed that many oligonucleotides are formed per preinitiation complex, including species as long as hexanucleotide and strikingly, the dinucleotide always represented $50 \%$ of the total of all oligonucleotides ${ }^{23}$. SSU RNAPs and DNA polymerases use the catalytic amino acid $\mathrm{K}$ at -8 position ${ }^{5,19}$ in contrast to R. Another interesting feature is the immediate upstream amino acid from catalytic $\mathrm{R}$ in both the of eubacterial and chloroplast $\beta$ subunits is the invariant $\mathrm{D}$. A long conserved stretch at the $\mathrm{C}$-terminal regions, i.e, exclusively at the end, is observed both in the eubacterial and chloroplast MSU RNAPs suggesting a possible role in polymerase cycling or termination. 


\section{CLUSTAL O (1.2.4) MSA of $\boldsymbol{\beta}$ ' subunits of eubacterial and chloroplast MSU RNAPs.}

SP I P0C506|RPOC1_ORYS SP |Q6ENI3।RPOC1_ORYNI sp|P16024|RPOC1 MAIZE SP $\mid$ A7M957|RPOC1 CUSRE SP | Q2MIA9|RPOC1 SOLLC SP|Q2VEI5IRPOC1_SOLTU sp|P11705|RPOC1 ${ }^{-}$SPIOL SP|P56763|RPOC1 ARATH SP|P46819|RPOC1 ${ }^{-}$SINAL Sp | Q85FM8|RPOC1 ADICA SP |Q85CL6 |RPOC1_ANTFO AEG34223.1

SP 1019897 |RPOC1 CYACA SD $104 G 3 A 6 \mid R P O C 1^{-}$EMTHU Sp | P42080|RPOC1 CYAPA sp|Q6B8R7|RPOC1_GRATL sp |Q1XDN6|RPOC1 PYRYE SP |P51251|RPOC1 DORPU sp |078484|RPOC1 ${ }^{-}$GUITH sp | B1X3M9|RPOC1 PAUCH Sp |P42079|RPOC1_SYNE7 SP | P14563|RPOC1 NOSCO ASR51305.1

OXR47930.1

SP |A7MQQ8|RPOC_CROS8 SP | B2TWH4 | RPOC_SHIB3

Sp |P0A8T7|RPOC ECOLI

tr|A0A237JUP3|A0A237JUP3 SHISO $\operatorname{tr} \mid A 0 A 0 F 1 R B F 2$ |AOAOF1RBF2 ENTAS $\operatorname{tr} \mid$ A0A1B3EWG0 |A0A1B3EWGO ENTCL tr|A0A0FOXM62|A0A0FOXM62_9ENTR sp|A9MHE9|RPOC SALAR

tr|A0A232XM43|A0A232XM43 SALMU

tr|B5RFK0|B5REKO SALG2

sp | POA2R5 |RPOC SĀLTI

tr|A0A078LHA5|Ā0A078LHA5 CITKO

tr|A0A0J2K6S7|A0A0J2K6S7 9ENTR

$\operatorname{tr|A0A0G3RZQ0|A0A0G3RZOO~KLEOX}$

$\operatorname{tr|A0A1R0FP41|A0A1R0FP41-CITBR}$

Sp | P0C506|RPOC1 ORYSJ

ORYN

Sp |P16024|RPOC1 MAIZE

SP |A7M957 |RPOC1_CUSRE

SP | Q2MIA9 |RPOC1 SOLLC

Sp |Q2VEI5 |RPOC1 SOITU

sp | P11705 |RPOC1 SPIOL

sp|P56763|RPOC1 ${ }^{-}$ARATH

sp |P46819|RPOC1 SINAL

SP | Q85FM8|RPOC1_ADTCA

Sp | Q85CL6 |RPOC1 ANTEO AEG34223.1

sp |019897|RPOC1 CYACA

sp |Q4G3A6 |RPOC1_EMIHU

Sp|P42080|RPOC1_CYAPA

Sp |06B8R7|RPOC1 GRATL

SP | Q1XDN6 |RPOC1 PYRYE

SP|R51251|RPOC1 PORPU

sp |078484|RPOC1 GUITH

Sp|B1X3M9|RPOC1 PAUCH

sp |P42079|RPOC1 SYNE7

SP 1 P14563|RPOC1 ${ }^{-}$NOSCO

ASR51305.1

OXR47930.1

sp |A7MQQ8 |RPOC_CROS8

sp | B2TWH4 |RPOC SHIB3

$\mathrm{sp} \mid \mathrm{POA8T7}$ |RPOC ECOLI

$\operatorname{tr} \mid$ A0A237JUP3|Ā0A237JUP3 SHISO

tr|A0A0F1RBF2 |A0A0F1RBF2_ENTAS $\operatorname{tr}|A 0 A 1 B 3 E W G 0| A 0 A 1 B 3 E W G 0$ ENTCL

tr|A0A0F0XM62|A0A0F0XM62_9ENTR

SP|A9MHE9|RPOC SALAR

tr|A0A232XM43|Ā0A232XM43_SALMU

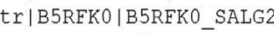

sp|P0A2R5 |RPOC SÄLTI

tr|A0A078LHA5|A0A078LHA5_CITKO tr|A0A0J2K6S7|AOA0J2K6S7 9ENTR tr|AOAOG3RZQO|AOAOG3RZQO KLEOX

tr|AOA1ROFP41|AOA1ROFP41_CITBR

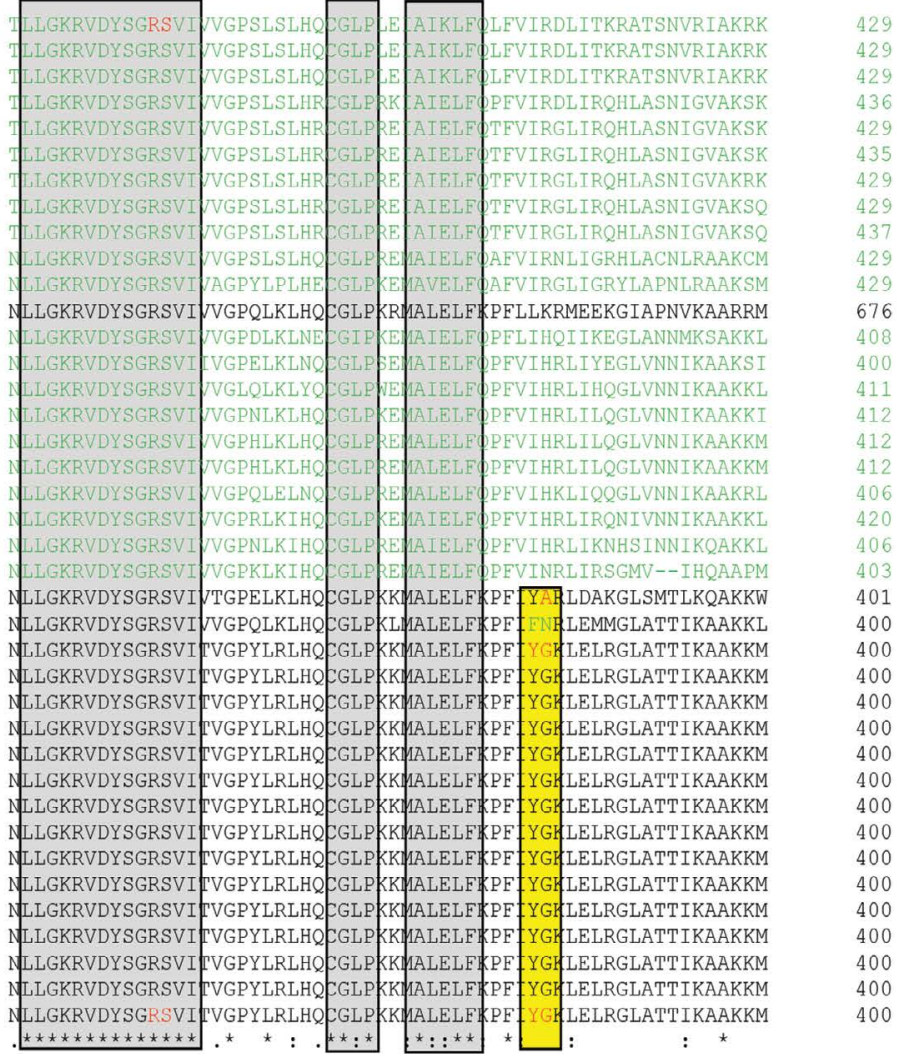

IWEKE---PIVWE ILOFVMRGH TINRAPTIHR GIOAFOPTLVEGRTI HPT

IWIKE--PIVTETLQEUVRG

TWEKE-- PTVWETLOEVMRGH IVTLNRAPTTHR GTOAFOPTLVEGRTI

IREKE---PIIWEILQEVMRGH VLINRAPTIHR GIQAFQPVLVEGHVT

IREKE---PIVWEILQEVMQGH VLINRAPTLHR GIQAFQPVLVEGRAI

IREKE---PIVWEILQEVMQGH VLLNRAPTLHR GIQAFQPVLVEGRAI

IREKE---PIVWKILOEVMOGH VLLNRAPTLHR GIOAFOPILVEGRAIC

TREKK--- PTVWETTOFVYMOGH UTIUNRAPTTHR GTOSFOPTTVEGRTT

IREKK---PIVWEILQEVMQGHIVLLNRAPTLHR GIQSFQPILVEGRTI

TRKGN---PI IWEVL,REVMRGH

IODKE---PI IWKILKEV IQGH

(1) CPAE

IQKNG---PLAWE ILANVMEGH ILINRAPTLHR GIQSFEPILVSGRAI LHPL CPAF

IQSND---PTIRDTLEEVT QNH VITINRAPTIHR S S IQAFEPTLVEGRAT

IQRNE---VVVWTVLEVIYGH

TOKNE---SSTWNVINEVTOGH VLINRAPTLHR GIOAFEPTLVEGRAI LHPI CPAP

IQKNE---SS IWNVLNEVIQGH VLLNRAPTLHR GIOAFEPILVEGRAI LHPL CPAE

IQKNE---LIVWNVLEEVIQGH ILINRAPTLHR GIQAFEPILVEGRAI

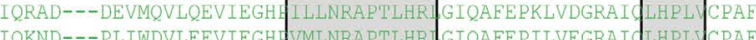

2RKND--OPLIWDVLEEVIES

VEKER---KEVWDILDEVIREHHVMLNRAPTLHRI G IQAFEPVLIEGKAI ILHPLVCSAE

VESQE---PVVWDILEEVIREH VMLNRAPT LHRI GIQAFEPVLIEGKAI LHPLY CAAF

VEREE---AVVWDILDEVIREH HLLNRAPTLHRI GIQAFEPVLIEGKAI LLHPLYCAAY

VEREE---AVVWDILDEVIREH HLLNRAPTLHRI GIQAFEPVLIEGKAIDLHPLYCAAY

VEREE---AVVWDILDEVIREHHVLLNRAPTLHRL GIOAFEPVLIEGKAI LHPLV CAAY

VEREE---AVVWDILDEVIREHHVLLNRAPTLHRI GIQAFEPVLIEGKA I LHPLYCAAY

VEREE---AVVWDILDEVIREH VLLNRAPTLHRIG GQAFEPVLIEGKAI I LHPLYCAAY

VEREE---AVVWDILDEVIREH ILLLNRAPTLHRI G IQAFEPVLIEGKAI ILHPLYCAAY

VEREE---AVVWDILDEVIREH VLLNRAPTLHRI G IQAFEPVLIEGKA I LHPLY CAAY

VEREE---AVVWDILDEVIREHHVLLNRAPTLHRI GIOAFEPVLIEGKAI LHPLVCAAY

VEREE---AVVWDILDEVIREH VLLNRAPT LHR G GIOAFEPVLIEGKAI L LHPL CAAY

VEREE---AVVWDILDEVIREH VLLNRAPTLHRIG GQAFEPVL IEGKAI I IHPLYCAAY

VEREE---AVVWDILDEVIREHHVLLNRAPTLHRI GIQAFEPVLIEGKAI d LHPLV CAAY

VEREE---AVVWDILDEVIREHHVLLNRAPTLHRIG IQAFEPVLIEGKAI LHPLYCAAY

VEREE---AVVWDILDEVIREHHVLLNRAPTLHRIG GOAFEPVLIEGKAI LHPLV CAAY

VEREE---AVVWDILDEVIREHH VLLNRAPTLHRIG GQAFEPVL IEGKAI LHPLVCAAY

VEREE---AVVWDILDEVIREH VLLNRAPTLHRIGIQAFEPVLIEGKAI LHPLYCAAY

$\left.: \quad *::::: * * * * * * * * *, *^{* *}::^{*} *^{*}: *^{*}:: * * * * *\right)$
29
29
29
36
29
35
29
29
37
29
29
676
08
00
11
12
12
12
06
20
06
03
01
00
400
00
00
00
00
00
400
00
00
00
00
400
00
00
00 
SP|P0C506|RPOC1 ORYSJ SP|Q6ENI3|RPOC1 ORYNI sp |P16024|RPOC1 MAIZE SP |A7M957 |RPOC1 CUSRE Sp/Q2MIA9|RPOC1 SOLLC SP |Q2VEI5 IRPOC1 SOLTU sp|P11705 |RPOC1 SPIOI SP|P56763|RPOC1 ARATH sp | P4 6819 | RPOC1_SINAL SP / Q85EM8 | RPOC1 ADICA Sp | Q85CL 6 | RPOC1 ANTFO AEG34223.1

SP|019897|RPOC1_CYACA sp |Q4G3A6 |RPOC1_EMIHU Sp|P42080|RPOC1 CYAPA sp |06B8R7|RPOC1 GRATI SP|Q1XDN6|RPOC1 PYRYE SP | P51251 | RPOC1 PORPU sp/078484|RPOC1 GUITH sp|B1X3M9|RPOC1 PAUCH SP|P42079 IRPOC1 SYNE Sp | P14563 | RPOCI ${ }^{-}$NOSCO ASR51305. 1

OXR47930.1

sp|A7MQQ8 |RPOC CROS8

sp|B2TWH 4 |RPOC SHIB

sp | P0A8T7 |RPOC ECOLI

$\operatorname{tr|A0A237JUP3|Ā0A237JUP3~SHISO~}$ $\operatorname{tr|A0A0F1RBE2|A0A0F1RBF2~ENTAS~}$

tr|A0A1B3EWG0|A0A1B3EWG0 ENTCL tr|A0A0F0XM62|A0A0F0XM62 9ENTR sp|A9MHE9 | RPOC SALAR

$\operatorname{tr}|A 0 A 232 \times M 43| \bar{A} 0$ A232XM43_SALMU tr|B5RFK0|B5RFK0 SALG2

sP|P0A2R5|RPOC SALTI

tr|A0A078LHA5|Ā0A078LHA5 CITKO

$\operatorname{tr|A0A0J2K6S7|A0A0J2K6S7~9ENTR~}$

$\operatorname{tr|A0A0G3RZQO|AOA0G3RZQ0-KLEOX}$

tr|A0A1R0EP41|A0A1R0EP41_CITBR

SP|POC506|RPOC1 ORYSJ

SP | Q6ENI3 | RPOC1 ORYNI

sp |P16024 | RPOC1 MAIZE

SPIA7M957|RPOC1 CUSRE

SP|O2MIA9|RPOC1 SOLLC

SP | Q2VEI5 | RPOC1 SOLTU

SP | P11705 |RPOC1_SPIOL

sp |P56763|RPOC1 ARATH

Sp|P46819|RPOC1 SINAI

SP 1085 FM 1 RPOC1 ADTCA

SP | Q 85CT.6 | RPOC1 ANTFO

AEG34223.1

Sp|019897|RPOC1 CYACA

SP|Q4G3A6 |RPOC1 EMIHU

SP|P42080|RPOC1 CYAPA

SP | Q 6B8R7 | RPOC1 GRATL

SP I Q1XDN 6 | RPOC1_PYRYE

SP|P51251|RPOC1 PORPD

Sp|078484|RPOC1 GUTTH

SD|B1X3M9|RPOC1 PAUCH

SP|P42079|RPOC1 SYNE7

SP | P1 4563| RPOC1 ${ }^{-}$NOSCO

ASR51305.1

sp |A7MQQ8 |RPOC CROS8

sp |B2TWH4 |RPOC_SHIB

SP|POA8T7|RPOC_ECOLI

$\operatorname{tr|A0A237JUP3|A0A237JUP3~SHISO~}$ tr|AOAOF1RBE2 |AOAOF1RBE2 ENTAS $\operatorname{tr} \mid \mathrm{A} 0 \mathrm{~A} 1 \mathrm{~B} 3 \mathrm{EWG}$ | A0A1B3EWGO ENTCL

$\operatorname{tr|A0A0F0XM62|A0A0F0XM62-9ENTR~}$ sp|A9MHE 9 |RPOC_SALAR

tr|A0A232XM43|Ā0A232XM43 SALMU tr|B5RFK0|B5RFK0 SALG2

SP|POA2R5 |RPOC SÄLTI

tr|A0A078LHA5|Ä0A078LHA5 CITKO

$\operatorname{tr|A0A0J2K6S7|A0A0J2K6S7-9ENTR~}$

tr|A0A0G3RZOO AOAOG3RZOO KLEOX tr|A0A1R0FP41|A0A1R0FP41_CITBR
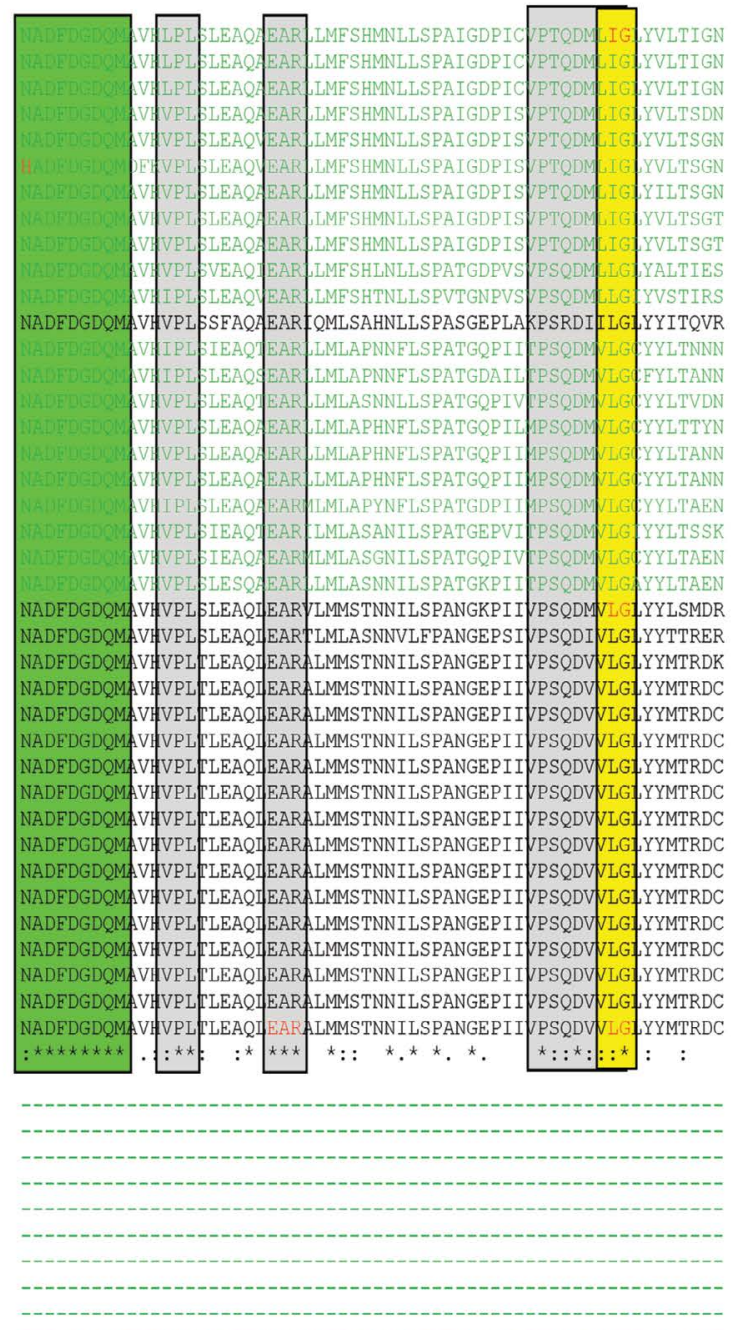

Figure 6 MSA of the $\beta$ ' subunits of eubacteria and chloroplast MSU RNAPs. 


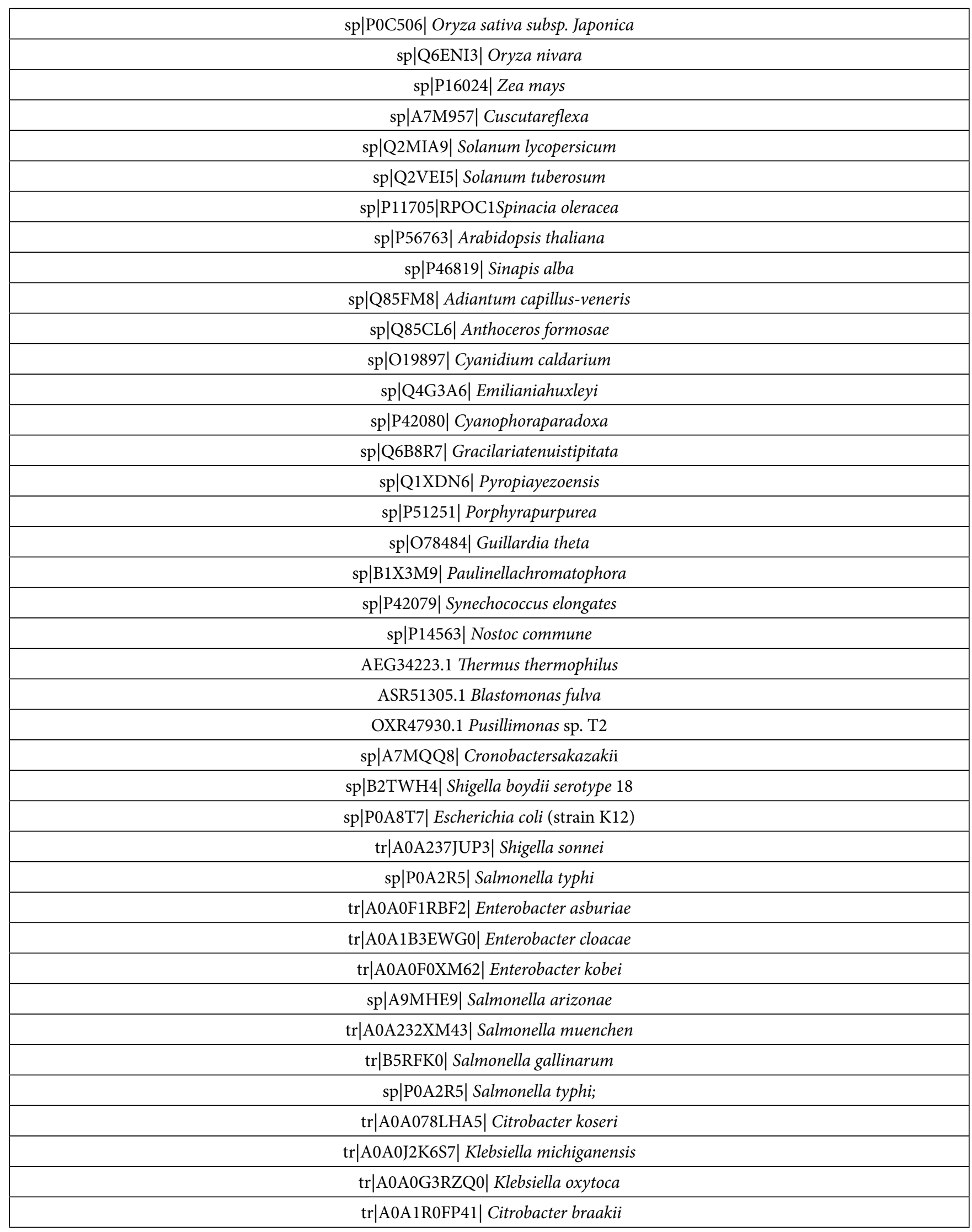




\section{CLUSTAL O (1.2.4) MSA of eubacterial $\beta$ ' and chloroplast $\beta$ "' subunits (Mix and Match analysis)}

ASR51305.1

SP |A7MQQ8 |RPOC CROS8

$\operatorname{tr}|A 0 A 0 F 1 R B F 2| \bar{A} 0$ A0F1RBF2_ENTAS

sp |B2TWH4 |RPOC SHIB3

SPIPOA8T7|RDOC ECOI

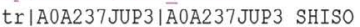

tr|A0A232XM43|A0A232XM43-SALMU

tr|B5RFK0 |B5RFKO_SALG2

sp|POA2R5 |RPOC_SÄLTI

$\operatorname{tr} \mid$ A0A0J2K6S7|A0A0J2K6S7 9ENTR

$\operatorname{tr|AOAOGBRZQ0|AOA0G3RZOO~KLEOX}$

$\operatorname{tr|A0A1R0FP41|A0A1R0FP41-CITBR}$

SP IQ85FM9 |RPOC2_ADICA

SP | B1VKH5 | RPOC2_CRYJA

SP|085C71|RPOC2 ANTFO

SDIPOC509/RPOC? ORYST

SD|P1 6025 IRPOC? MATZFE

sp|Q6L3A5|RPOC2 SACHY

Sp|Q01923|RPOC2_SORBI

sp | P56764|RPOC2 ARATH

SP|P11704 IRPOC2 SPTOI

SPIO2MIBO |RPOC2 SOLIC

SplQ0ZJ30|RPOC2 VITVI

sp |B1X3M8 |RPOC2 PAUCH

SP |P48120|RPOC2 CYAPA

ASR51305.1

sp |A7MQQ8 |RPOC_CROS8

tr|A0A0F1RBF2|A0A0F1RBF2 ENTAS

sp|B2TWH4 |RPOC SHIB3

SP|POA8T7/RPOC ECOT

$\operatorname{tr} \mid A 0 A 237$ JUP $3 \mid \bar{A} 0$ A237JUP3_SHISO

tr|A0A232XM43|A0A232XM43_SALMU

tr|B5RFK0|B5RFK0 SALG2

Sp|P0A2R5|RPOC SALTI

tr|A0A0J2K6S7|Ā0A0J2K6S7 9ENTR

tr|AOAOG3RZOO IAOAOG3RZOO KLEOX

tr|A0A1R0FP 41|A0A1R0FP41_CITBR

sp | Q85FM9|RPOC2_ADICA

sp |B1VKH5 |RPOC2 CRYJA

SP $1085 \mathrm{C} 71 \mid$ RPOC2 ANTFO

SP|POC509|RPOC2 ORYS

Sp |P1 6025 |RPOC2_MAIZE

SPIQ6L3A5 |RPOC2_SACHY

splQ01923|RPOC2 SORBI

SP|P56764|RPOC2 ARATH

SP|P11704|RPOC2 ${ }^{-}$SPIOL

SPIQ2MIBO |RPOC2 SOLLC

SP IQOOZJ30 |RPOC2_VITVI

sp |B1X3M8 | RPOC2 PAUCH

sp|P48120|RPOC2 CYAPA

ASR51305.1

Sp|A7MQQ8 |RPOC CROS 8

tr|A0A0F1RBE2|A 0 A0F1RBF2 ENTAS

Sp |B2TWH4 |RPOC SHIB3

SP | POA8T7 |RPOC ECOL

tr|A0A237JUP3|A0A237JUP3_SHISO

tr|A0A232XM43|A0A232XM43_SALMU

tr|B5RFK0|B5RFK0 SALG2

SP|POA2R5 |RPOC SALLTI

tr|A0A0J2K6S7|ĀAOA0J2K6S7_9ENTR

tr|AOAOG3RZQOIAOAOG3RZQO KLEOX

tr|A0A1R0FP 41|A0A1R0FP41 CITBR

splQ85EM9|RPOC2 ADICA

sp|B1VKH5 |RPOC2 CRYJA

SP | Q85C71|RPOC2 ANTFO

SP|POC509|RPOC2_ORYS J

SP|P16025 |RPOC2 MAIZE

SPIQ6L3A5 IRPOC2 SACHY

SPIO01923 IRPOC2 SORBI

SP|P56764|RPOC2 ARATH

SP|P1.1704 |RPOC2 SPIOL

SPIQ2MIBO |RPOC2 SOLLC

SPIQ0ZJ30|RPOC2 VITVI

SP|B1X3M8 |RPOC2 PAUCH

Sp | P48120|RPOC2 CYAPA

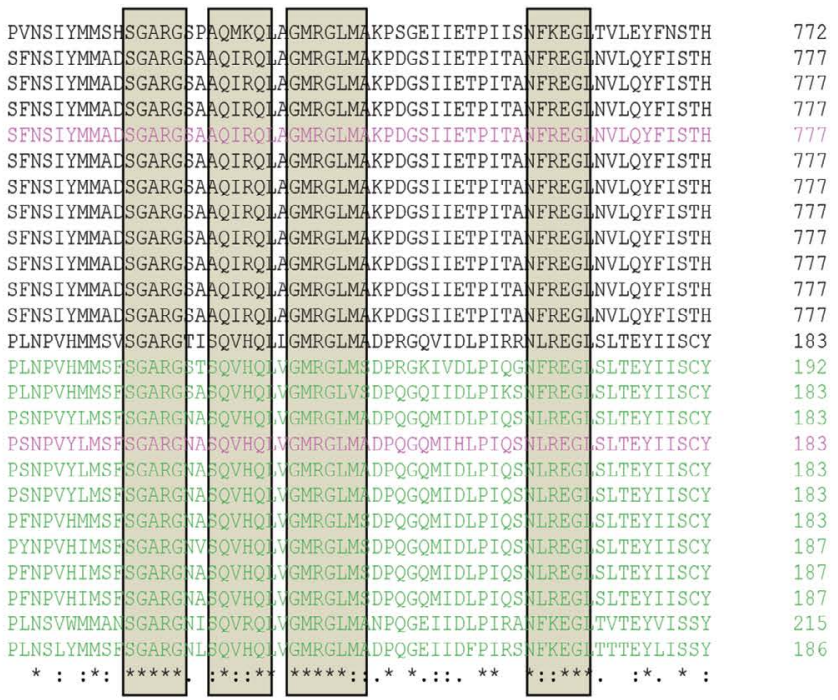

GARKGL PTALKTANSGYLTRRLVDY SQDCVIVQEDCGTENALEMRAIVQ----GGSVIA GARKGLADTALKTANSGYLTRRLVDYAQDLVVTEDDCGTLEGITMTPVIE----GGDVK GARKGL DTALKTANSGYLTRRLVDYAODLVVTEDDCGTLEGITMTPVIE----GGDVKE GARKGL DTALKTANSGYLTRRLVDYAQDLVVTEDDCGTHEGIMMTPVIE----GGDVKE

GARKG GARKGL DTALKTANSGYLTRRLVDYAODLVVTEDDCGTHEGIMMTPVIE----GGDVKE GARKGL DTALKTANSGYLTRRLVDYYQDDLVVTEDDCGTHEGILMTPVIE----GGDVKE GARKGLADTALKTANSGYLTRRLVDYAODLVVTEDDCGTHEGILMTPVIE-----GGDVKE CARKGL DTALKTANSGYLTRRLVDVAODLVVTEDDCGTHEGILMTPVIE----GGDVKE GARKGT DTAT TTANSCYT TRRIVDWAODTVVTEDDCCTIEGITUTPV GARKGL DTALKTANSGYLTRRLVDYAQDLVVTEDDCGTLEGITMTPVIE----GGDVKE

GARKGV TAVRT SDAGYLTRRLVE VOHIAVRRRDCETPRS LAFLT SNT GERRRGELG'T

ISIRTADAGYLTRRLVE VQHLVVRKVICGT OGLFVNLI---QDRETIKNK

RRGY TAVRTSDAGYLTRRIVE VOHIVVRKTDCNTERGISINST---EDKKKNLO

RKGV TAVRTADAGYLTRRLVE VQHI IVRRRDCGTIQAISVSPQ---N---GMTE

PRKG

RKGV TAVRTADAGYLTRRLVE VOHIIVRRRDCGTIQGISVSPQ---N---GMTE

TAVRTSDAGYLTRRT,VE VOHTVVRRTDCGTTRGTSVSPR---NKNRMMSFR

DTAVRTSDAGYLTRRLVE VOHIVVRRRDCGTIRGISVSPQ---N--STMPE

KGV TAVRTSDAGYLTRRLVE VQHIVVRRTDCGTARGISVSPR---N--GIMPER KG) TAVRTSDAGYLTRRLVE VQHIVVRRTDCGTIRGISVSPR---N--GMIPEA GARKGV DTALRTADSGYLTRRLVD AQEVIIREIDCETPRGVILTAL---K-ENGKILI

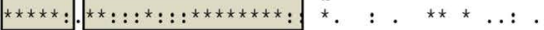

SLGERILGRTLAEDISDAKTGEIVIAKDTLLDEANIATIEA------LGLQAARFRPLI PLRDRVLGRVTAEDVIKPGTADILVARNTLLNEQWCDILEA------NSVDSVK YR PLRDRVLGRVTAEDILKPGTADILVPRNTLLHEHWCDLLEA-------NSVDSVKVR RVVS PLRDRVLGRVTAEDVLKPGTADILVPRNTLLHEOWCDLLEE------NSVDAVK /R RVVS PLRDRVIGRVTAEDVLKPGTADILVPRNTLLHEQWCDLIEE------NSVDAVK RVVB PLRDRVLGRVTAEDVLKPGTADILVPRNTLLHEQWCDLIEE------NSVDAVK N R SVVS PLRDRVLGRVTAEDVLKPGTADILVPRNTLLHEQWCDLLEA------NSVDAVKYRBVVS PLRDRVLGRVTAEDVLKPGTADILVPRNTILHEOWCDLIEA------NSVDAVK YR EVVS PLPDRVIGRVIAEDVLKPGTADILVPRNTLLHEOHCDLLEA----NSVDAVK PLRDRVLGRVTAE DVLKPGTADILVPRNTLLHEHWCDLLEE------NSVDSVK K R PVVS PLRDRVLGRVT'AEDVLKPGTADILVPRNTLLHEHWCDLLEE-------NSVDSVKYR RVVS PLRDRVLGRVTAEDVLKPGTADILVPRNTLLHEQWCDLLEE------NSVDSVKVRRVVS MPHOGLVGRVLADHVYWD--VRCIATRNODISDGLASNIMA------SSOPIH RPI FVLOTLIGRVIADOVYTH--GBCTATRNEDTGIKLANOLYY-_-_-_FOVOPTY P PPET

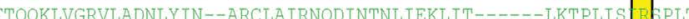
LFVQTLIGRVLANDIYIG--SRCIATRNQDIGIGLVNRFITTERAQPFRAQPIY PPF LEVQTLIGRVLADDIYIG--SRCIASRNQDIGIGLVNRFITAFRAQPFRAQPIY R PPF LEVOTLIGRVLADDIYIG--SRCIASRNQDIGIGLVNRFITAERAQPFRAOPIY BPF

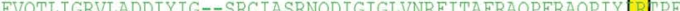
TFTOMTTGRVLADDTYTG--SRCVAFRNODTGTGTVNRT TT----_-_FGTOSTS R PPFC LTOTLTGRVT,ADDTYMG--SRCTATRNODTGVGIVNRFTT------TRTQLTS R PPF IESQTLIGRVLADDIYMG--SRCIATRNQAIGIGLVNRFIT------FRAQPIS R PPEI IFIQTLIGRVLADDIYMG--PRCIAIRNQDIGIGLVNREIT-----FQAQTIS RPPET -YGTKLVGRLAAQSVVVDNENRVIVRRNREIDLITACRIEA-----ADIPSVI R PPLI SLKDRLVGRVLLHNLYHPKTHSLIAQKNES ISVLLADDIIK-----AGLKEVW R PPIT

72
777
777
77
777
77
77
77
77
777
77
777
183
92
183
183
183
83
183
183
187
187
187
15


ASR51305.1

SP|A7MQQ 8 |RPOC_CROS8

tr|A0A0F1RBF2| $\bar{A} 0 A 0 F 1 R B F 2$ ENTAS

sp|B2TWH4 |RPOC SHIB3

SPIPOABT7|RPOC ECOLI

$\operatorname{tr} \mid A 0 A 237$ JUP $3 \mid \bar{A} 0$ A237JUP 3 SHISO

tr|A0A232XM43|A0A232XM43_SALMU

tr|B5RFKO|B5RFK0_SALG2

SP|P0A2R5|RPOC SÄLTI

$\operatorname{tr}|A 0 A 0 J 2 K 6 S 7| A 0 A 0 J 2 K 6 S 7$ 9ENTR

$\operatorname{tr|A0A0G3RZQO|A0A0G3RZQ0-KLEOX}$

tr|A0A1R0FP41|A0A1R0FP41_CITBR

sp | Q85 FM9 | RPOC2 ADICA

sp|B1VKH5 |RPOC2 CRYJA

sp I Q85C71 | RPOC2 ANTFO

SP|P0C509|RPOC2 ORYSJ

SP|P16025|RPOC2 MAIZE

SPIO6L3A5|RPOC2 SACHY

sp | Q01923 | RPOC2_SORBI

sp | P56764 | RPOC2 ${ }^{-}$ARATH

SP|P11704 |RPOC2 SPIOI

SP | Q2MIBO | RPOC2 SOLLC

sp | QOZJ30 | RPOC2 ${ }^{-}$VITVI

$3 \mathrm{P} \mid \mathrm{B} 1 \times 3 \mathrm{M} 8$ |RPOC2 PAJCH

SP|P48120|RPOC2 CYAPA

ASR51305.1

sp|A7MQQ8 |RPOC CROS8

tr|A0A0F1RBF2 | A $0 A 0 F 1 R B F 2$ ENTAS

sp|B2TWH4 |RPOC SHIB3

SP|POABT'7|RPOC ECOL

$\operatorname{tr|A0A237JUP3|\overline {A}0A237JUP3~SHISO~}$

tr|A0A232XM43|A0A232XM43_SALMU

tr|B5RFK0|B5RFK0 SALG2

SP|POA2R5|RPOC SĀLTI

tr|A0A0J2K6S7|Ā0A0J2K6S7_9ENTR

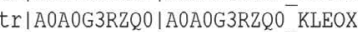

tr|A0A1R0FP41|A0A1R0FP41 CITBR

sp | Q85FM9 | RPOC2 ADICA

SP IB1VKH5 | RPOC2_CRYJA

sp/Q85C71|RPOC2 ANTFO

sp |P0C509|RPOC2 ORYSJ

SP|P16025|RPOC2 MAIZE

sp I Q6L3A5 | RPOC2_SACHY

sp|Q01923|RPOC2 SORBI

SP|P56764 |RPOC2 ARATH

SP |P11704 | RPOC2 SPIOI

SPIQ2MIBO IRPOC2 SOLLC

sp|Q0ZJ30|RPOC2 VITVI

SP|B1X3M8|RPOC2 PAUCH

SP|P48120|RPOC2_CYAPA

ASR51305.1

SP |A7MQQ8 8 RPOC_CROS8

$\operatorname{tr|A0A0F1RBF2|A0A0F1RBF2~ENTAS~}$

sp |B2TWH4 |RPOC SHIB3

SP|POA8T 7 IRPOC ${ }^{-}$ECOL

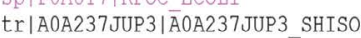

$\operatorname{tr|A0A232XM43|A0A232XM43~SALMU~}$

$\operatorname{tr|B5RFK0|B5REK0~SALG2~}$

SP|POA2R5|RPOC SÄLTI

tr|A0A0J2K6S7|Ā0A0J2K6S7_9ENTR

tr|A0A0G3RZQ0|AOAOG3RZQO KLEOX

tr|A0A1R0FP41|A0A1R0FP41_CITBR

Sp | Q85FM9 | RPOC2_ADICA

sp |B1VKH5 | RPOC2 CRYJA

sp $1085 \mathrm{C} 71 \mid$ RPOC2 ANTFO

sP|POC509|RPOC2 ORYSU

SP|P16025|RPOC2 MAIZE

SPIO6L3A5।RPOC2 SACHY

Sp|001923|RPOC2 SORBI

sp | P56764 |RPOC2_ARATH

SP |P11704 | RPOC2_SPIOL

SP $\mid$ OLMIBO | RPOC2 SOLLC

SP|Q0ZJ30|RPOC2 VITVI

SP IB1X3M8 | RPOC2_PAUCH

SP|P48120|RPOC2 CYAPA

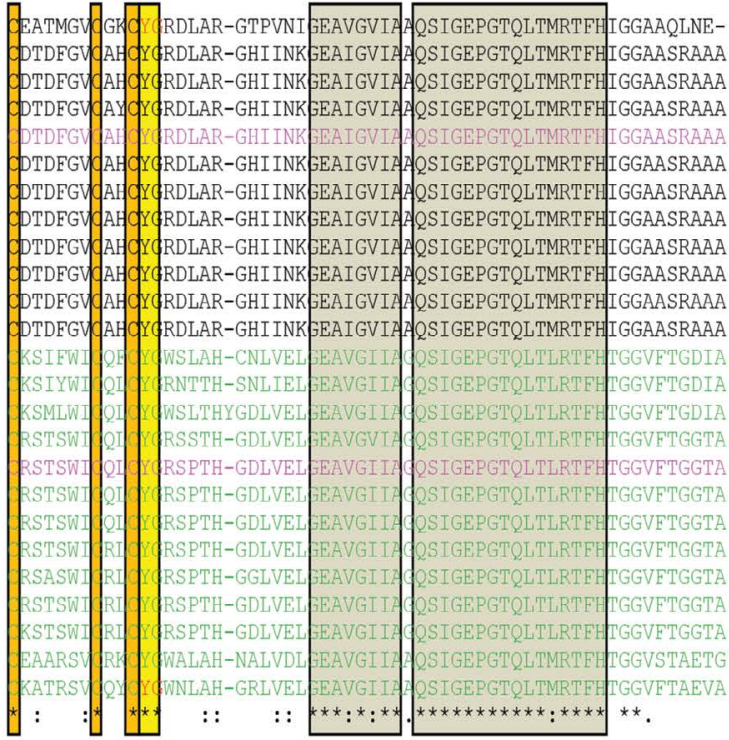

940

946

946

946

946

946
946

946

946

946

946

946

353
360

360
352

354

354

354

351
351

353

353

353
379

355

GETPRTAELFEARKPKDNAIIAKVSGRIEFVRDYKAKRKTAIHPEEG-ESVEYLVPK--S GGLPRYADLFEARRPKEPAILAEVAGIVSFGKETKGKRRLVITPVDGGDAYEEMIPK-GGLPRYADLFEARRPKEPAILAEISGI ISFGKETKGKRRLVITPVDGSEPYEEMIPK--W GFLPRYADLFEARRPKEPAILAE ISGIVSFGKETKGKRRLVITPVDGSDPYEEMIPK--

- PR ADLFEARRPKEPAILAEISGTVSFGKETKGKRRUVITPVDGSDPYEFMTPK-GGLPRYADLFEARRPKEPAILAEISGIVSFGKETKGKRRLVITPVDGSDPYEEMIPK--W GLPRY ADLFEARRPKEPAILAEIAGIVSFGKETKGKRRLVITPVDGSDPYEEMIPK--W GGLPRY ADLFEARRPKEPAILAEIAGIVSFGKETKGKRRLVITPVDGSDPYEEMIPK--W GLPRTADLFEARRPKEPAILAEIAGIVSFGKETKGKRRLVITPVDGSDPYEEMIPK-GGLPRYADLFEARRPKEPAILAE ISGIISFGKETKGKRRLVITPVDGSDPYEEMIPK-GLPRVADLFEARRPKEPAILAE ISGIISFGKETKGKRRLVITPVDGSDPYEEMIPK-GGIPRI ADLFFARRPKEPATLAFTSGT ISFGKETKGKRRLVITPVDGSEPYFFMT PK--

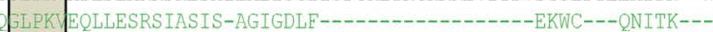

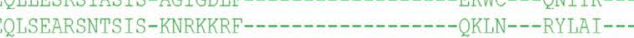

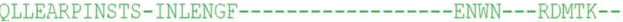
QIFEARSIDSLS -PNLERRI-----------------EDWN---ERTPR--QTFEARSTDSLS-PNLERRT---_-_-

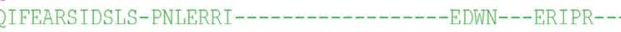
QIFEARSIDSLS - PNLERRI----------------EDWN---ERTPR--

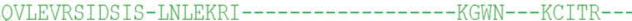
OVLEVRSIDS IS - INLEKRI-.-_-_QVLEVRSIDSIS-MNLEKRV--_-_-_-

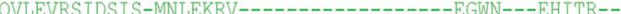
PELTEARRPRDSAVLCKOPGVLTTRHDEETDSS--TAVTKSANGEETEYPIULG ELLEARKPKDNCVLSTHPGETQLYYSETIELK--IKSLDKIN---TLLELQPG $:: * *$ *

VSETOEVYRLOGVKI NDKHIEVIVROMLOKVEIT-----------DGGDTTLLPGEQLDR VNEVQDVYRLQGVKI NDKHIEVIVRQMERKATIM--------DAGSSEFLEGEQVEY TNEVQDVYRLQGVKINDKHIEVIVROM RKATIE-----------NAGSSEFIEGEOVEY VNEVQDVYRLQGVKINDKHIEVIVROMLRKATIV-----------NAGSSDELEGEQVEY

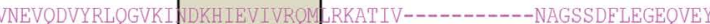
VNEVQDVYRLQGVKI NDKHIEVIVRQM RKATIV---------NAGSSDELEGEQVEY NEVQDVYRIOGVKINDKHIEVIVROM RKATIE--------SAGSSDELEGEQVEY VNEVQDVYRLOGVKINDKHIEVIVROMLRKATIE-----------SAGSSDFLEGEOVEY VNEVQDVYRLQGVKI NDKHIEVIVRQMERKATIE----------SAGSSDFLEGEQVEY VNEVQDVYRLQGVKINDKHIEVIVRQMERKATIE-----------SAGSSDELEGEQVEY VNEVQDVYRLQGVKI NDKHIEVIVROM RKATIE------------SAGSSDELEGEQVEY VNEVODVYRLOGVKINDKHIEVIVROMLRKATIE--_-_-_-_-_SAGSSDFLEGEOVEY IDQIRKVYESQGVRI DKHIEIIVRQI SRVVAS---------EDGVTNVELPGELVEI VDEIOKVYRSQGVQI DKHIEIIVROI SKVLVVDVDRSENKNWENGLGSLELPGELVGI

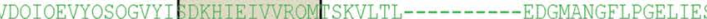
TNKIOKVYRSQGVQI INRUIIEIIIROV SKVRVS-_-_-_EDGMSNVESPGELIGI JNKIQKVYRSQGVQI IINRHTEIITRQVESKVRVS---------EDGMSNVELPGELIGI

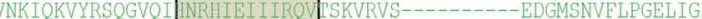

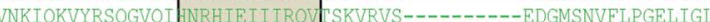
NNIOKVYRSQGVQI INRHIEIIVRQI SKVLVS--.--EEGMSNVELPGELIGL VNKIQKVYRSQGVQI INRHIEIIVRQI SKVLVS----------EDGMSNVELPGELIGI UNKLOOVYRSOGVQI INRH

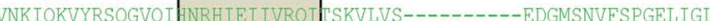
VTEVONVYKSOGVTI INKHIEVIVROM SKVRIE---_-_---DAGETTLLPGELIE VSEVQSVYQSQGIDT DDKHTEITIKQM NNKVIE-----------EGGDTTLIPNELIEH

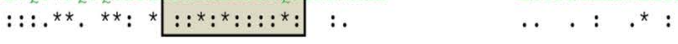


ASR51305.1

sp |A7MQQ8 |RPOC CROS8

$\operatorname{tr} \mid$ AOAOF1RBE2 | $A$ AAOF1RBF2_ENTAS

sp | B2TWH4 | RPOC_SHIB3

SO IPOA8T7 IRPOC ECOL

tr|A0A237JUP3|Ā0A237JUP3_SHISO

$\operatorname{tr} \mid$ A0A232XM43|A0A232XM43-SALMU

$\operatorname{tr} \mid$ B5RFKO | B5RFKO_SALG2

sp|P0A2R5 |RPOC SÄLTI

$\operatorname{tr} \mid$ A0A0J2K6S7|AA0A0J2K6S7 9ENTR

tr|AOAOG3RZQO |AOAOG3RZQ0- KLEOX

tr|A0A1R0FP41|A0A1R0FP41 ${ }^{-}$CITBR

Sp | Q85FM9 | RPOC2_ADICA

Sp | B1VKH I RPOC2 ${ }^{-}$CRYJA

sp Q Q85C71 | RPOC2 ANTFO

sp | POC509 | RPOC2_ORYSJ

SP|P16025 RPOC2 MAIZE

Sp | Q6L3A5 |RPOC2_SACHY

sp| Q01923| RPOC2 SORBI

sp|P56764| RPOC2 ARATH

Sp | P11704 | RPOC2_SPIOL

SD / Q2MIBO | RPOC2 SOLLC

Sp | Q0ZJ30 | RPOC2 VITVI

sp | B1X3M8 | RPOC2 ${ }^{-}$PAUCH

sp | P48120 | RPOC2_CYAPA

ASR51305.1

SP |A7MQQ8 |RPOC_CROS8

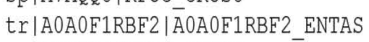

sp|B2TWH4 |RPOC SHIB3

SP | POA.8T7 |RPOC ECOLI

$\operatorname{tr} \mid$ A0A237JUP3|Ā0A237JUP3 SHISO

tr|A0A232XM43|A0A232XM43_SALMU

tr|B5RFKO|B5RFKO SALG2

Sp|P0A2R5 |RPOC SALTI

tr|A0A0J2K6S7|Ā0A0J2K6S7 9ENTR

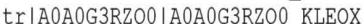

tr|A0A1R0FP41|A0A1R0FP41_CITBR

sp | Q85FM9 | RPOC2_ADICA

sp|B1VKH5|RPOC2 CRYJA

$\mathrm{SP}|\mathrm{Q} 85 \mathrm{C} C 71| \mathrm{RPOC2}$ ANTFO

sp|P0C509 |RPOC2 ORYSJ

sp | P16025 | RPOC2 MAIZE

Sp | Q6L3A5 |RPOC2 SACHY

sp | Q01923|RPOC2 SORBI

SP | P56764 | RPOC2 ARATH

sp|P11704 |RPOC2 SPIOI

Sp | Q2MIBO | RPOC2 ${ }^{-}$SOLLC

sp | Q0ZJ30 | RPOC2 VITVI

sp | B1X3M8 | RPOC2 PAUCH

SP $\mid$ P48120 | RPOC2 CYAPA
EEMDEYNGRLDAGKK-FAEGKPV_LGITKASLQTRSS IAAASFQETTRVLTQAAVEGKRD SRVKIANRELEANGKVSATYMRDILGITKASLATESS ISAASFQETTRVLTEAAVAGKRD SRVKIANRELEANGKIGATESRD_LITIKASLATESEI AAASFQETPRVLTEAAVAGKRD SRVKIANRELEANGKVGATYSRDLLIGITKASLATESEI SAASEQTT RVLTEAAVAGKRD SRVKIANREIEANGKVGATYSRD. LGITKASLATS SEI A ASEQET TRVLTEAAVAGKRI SRVKIANRELEANGKVGATYSRD_LGITKASLATESEIAAASFQETTRVLTTEAAVAGKRD SRVKIANRELEANGKVGATFSRDILGITKASLATESFIAAASFQETTRVLTEAAVAGKRD SRVKIANRELEANGKVGATESRD LIGITKASLATESEI AAASFOET PRVLTEAAVAGKRD SRVKIANRELEANGKVGATFSRD ILGITKASLATESSFIAAASFQETTRVLTEAAVAGKRD SRVKIANRDLEANGKVGATFSRD_LGITKASLATESFISAASFOETTRVLTEAAVAGKRD SRVKIANRDLEANGKVGATFSRD_LGITKASLATTESFISAASFQET TRVLTEAAVAGKRD SRVKIANRDLEANGKVGATFSRDALGITKASLATESFI A ASEQEET TRVLTEAAVAGKRD

SQAERINRVIKK----SIFYEPI LGMTR SSLST SELA A AEQE IRVIAKAALRGRID

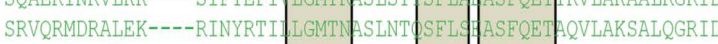
SRAORMNRVIEE----SVLYKPI IGITKASLNT SEI HASFOEI TRVIAKAALRGRID LRAERAGRALDE----SIYYRAI LGITR ISLNI SEI A ASEQE ERVLAKAALRGRID URAERAGRALDE----SIYYRAI IGITRASLNT SEIA ASEOETARVLAKAALRGRID IRAERAGRALDE---SIYYRAI LGITE SLINT SEI A ASEQE RVIAKAALRGRID LRAERAGRALDE----SIYYRAI IGITRASLNT SEI ASEQETARVIAKAALRGRID IRAERTGRALEE----AICYRAV LGITRASLNT SEIA ASEOET TRVLAKAALRGRID ERAERTGRALEE----AICYRAT LGITR SSLNT SEI A ASFQE ARVLAKAALRGRID LRAERMGRALEE----AICYRVV LGITR ASLNT SEI A ASEOE ARVLAKAALRGRID IRAERTGRAFEE----AICYRTI LGITRASLNT SEI A ASEQE IRVLAKAALWGRID RHVEKVNKAMFMTSSSPAVEIPE LGITK SLINT SEI ASEQET TRVITEAAIEGKTD QQIEKMNEKFSTSNGQLASYTPI LGITRSSLNT SEI A ASFOET TRVIAKAAVEGKID

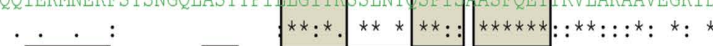
SL GLKENVIVGRLIPA GTGAGMI RMRIAANSRDAALR--AQQRAL-QRAMA------- 1377 ELRGLKENV IVGRLIPA GTGYAYHQDRMRRRAAGELPA--APQ------------ 1383 ELKGLKENV FVGRLIPAGTGYAYYQDRMRRRAAGELPA--APQ--------------- 1383 ELRGLKENV IIVGRLIPA GTGYAYHQDRMRRRAAGEAPA--APQ--------------- 1383 EI GLKENV VGRLIPA ET YAYY QDRMRRRAAGEAPA-- -APQ------------- 1383 ELRGLKENV IVGRLIPA GTGYAY QDRMRRRAAGEAPA--APQ---------------- 1383 EL KGLKENVIVVGRLIPA GTGYAYYQDRMRRRAAGEQPA--TPQ-------------- 1383 ELKGLKENVEVGRLIPA GTGYAYYQDRMRRRAAGEQPA--TPQ--------------- 1383 ELRGLKENV EVGRLIPA GTGYAY QDRMRRRAAGEQPA--TPQ--------------- 1383 ELRGLKENV IVGRLIPA GT GYAYYQDRMRRRAAGELPA- -APQ--------------- 1383 ELRGLKENV TVGRLIPA GTGYAYHODRMRRRAAGELPA-- -APO--------------EL RGLKENV IVGRLIPAGTGYAY LDRMRRRAAGELPA_-APQ--------------- 1383 WI GLKENV IGDSVPY GI SPEIYCQININKEKESRLASGG-SKKLTKWETGSSISGYY 1393 WL KIKENV LGGMIPV

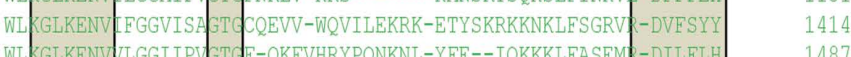
WI GLKENV LGGIIPV GTCE-QKEVHRYPQNKNL-YEE--IQKKKLEASEM -DILFLH $148^{7}$

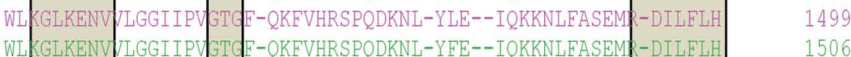
WI GIKENV ULGIIPY E-QKEVHRSPQDKNI-YFE--IKKKNIFASEM -DILFLH 1492 WL GLKENV LGGVIPA WI KGLKENV LGGMIPY ID E-KGEVHHSSQHKDI-PLK--TKKQNLEEGEM -DILFYH 1350 WI KGLKENV ILGGVIPY IT E-KGLVHPSKQHNNI-DLE--TKKTNLEEGEM -DILEHH WL GGLKENV LGGMIPY OTE-KGLVHRSRQHNNI-PLEMETKKNNLEEREM -DILEHH 1368 YI GIKENV IGRIIPA ET ESGEEEELRSEAGPHPDILD---------- EDPAGYR 1288

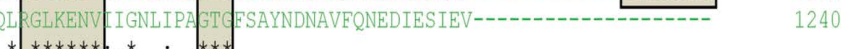

1328

342

1342

1342

1342

1342

(3)

1342

342

1100

357

444

1451

302

1295

311

41 $\underbrace{228}_{2.0}$ 3 83 83 83 3

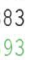
51 4 99 2 sio .

Figure 7. MSA of eubacterial $\beta$ ' and chloroplast $\beta$ " subunits of MSU RNAPs.

\begin{tabular}{|r|}
\hline ASR51305.1 Blastomonas fulva \\
\hline sp|A7MQQ8|RPOC_CROS8 Cronobactersakazakii \\
\hline $\operatorname{tr|} \mid$ A0A0F1RBF2|A0A0F1RBF2_ENTAS Enterobacter asburiae \\
\hline sp|B2TWH4|RPOC_SHIB3 Shigella boydii \\
\hline sp|P0A8T7|RPOC_ECOLI Escherichia coli, K12 \\
\hline $\operatorname{tr} \mid$ A0A237JUP3|A0A237JUP3_SHISO Shigella sonnei \\
\hline $\operatorname{tr|} \mid$ A0A232XM43|A0A232XM43_SALMU Salmonella muenchen \\
\hline tr|B5RFK0|B5RFK0_SALG2 Salmonella gallinarum \\
\hline sp|P0A2R5|RPOC_SALTI Salmonella typhi \\
\hline
\end{tabular}




\begin{tabular}{|c|}
\hline $\operatorname{tr|} \mid$ A0A0J2K6S7|A0A0J2K6S7_9ENTR Klebsiella michiganensis \\
\hline $\operatorname{tr|} \mid$ A0A0G3RZQ0|A0A0G3RZQ0_KLEOX Klebsiella oxytoca \\
\hline $\operatorname{tr} \mid$ A0A1R0FP41|A0A1R0FP41_CITBR Citrobacter braakii \\
\hline sp|Q85FM9|RPOC2_ADICA Adiantum capillus-veneris (fern) \\
\hline sp|B1VKH5|RPOC2_CRYJA Cryptomeria japonica (conifer) \\
\hline sp|Q85C71|RPOC2_ANTFO Anthoceros formosae (hornwort) \\
\hline sp|P0C509|RPOC2_ORYSJ Oryza sativa \\
\hline sp|P16025|RPOC2_MAIZE Zea mays \\
\hline sp|Q6L3A5|RPOC2_SACHY Saccharum hybrid \\
\hline sp|Q01923|RPOC2_SORBI Sorghum bicolor \\
\hline sp|P56764|RPOC2_ARATH Arabidopsis thaliana \\
\hline sp|P11704|RPOC2_SPIOL Spinacia oleracea \\
\hline sp|Q2MIB0|RPOC2_SOLLC Solanum lycopersicum \\
\hline sp|Q0ZJ30|RPOC2_VITVI Vitis vinifera \\
\hline sp|B1X3M8|RPOC2_PAUCH Paulinellachromatophora \\
\hline sp|P48120|RPOC2_CYAPA Cyanophoraparadoxa
\end{tabular}

\subsection{MSA of the Eubacterial $\beta^{\prime}$ Subunits with $\beta$ ' Subunits of Chloroplasts in the MSU RNAPs (Mix and Match Analysis)}

Figure 6 shows the 'mix and match' MSA analysis of $\beta$ ' subunits of eubacterial and chloroplast MSU RNAPs. It is clear from the figure that the metal binding site is absolutely conserved in both the $\beta$ ' subunits of both the MSU RNAPs (highlighted in green). The template/NTP binding YG pair and its catalytic $\mathrm{R}$ placed at -16 is seen only in the $\beta$ ' subunits of eubacteria (highlighted in yellow) and conspicuously absent in the $\beta$ ' subunits of chloroplast MSU RNAPs. However, it is interesting to note that this important catalytic motif is found in the $\beta$ " subunits of the chloroplast MSU RNAPs (Figure 7). The C-terminal ends of $\beta$ ' subunits of eubacteria are highly conserved with the consensus sequence of - GLGGSDND - (highlighted) but no such conservation is seen in the chloroplast $\beta^{\prime}$ subunits (data not shown). The mix and match analysis have revealed that the thermophilic RNAP aligns with the plant RNAPs rather than with the eubacterial RNAPs.

\subsection{MSA of the Eubacterial $\beta$ ' Subunits with the $\beta$ " Subunits of Chloroplasts (Mix and Match Analysis)}

Figure 7 shows the 'mix and match' MSA analysis of $\beta$ ' and $\beta^{\prime \prime}$ subunits of eubacterial and chloroplast MSU RNAPs. There are large numbers of conserved motifs and some are found to be long stretches (highlighted) and a small number of diads and triads among them, as seen in the case of $\beta^{\prime}-\beta^{\prime}$ matching. The catalytic, template and substrate binding motifs are highlighted in yellow. The possible metal binding amino acids are highlighted in green. The E. coli $\beta$ ' and maize $\beta$ " subunits are used as the standard for numbering and highlighted in magenta. The $\mathrm{Zn}$ binding motif (from the crystallographic data of E.coli ${ }^{24}$ and Thermus aquatics ${ }^{20}$ MSU RNAPs is shown in orange. Interestingly, it is completely conserved in both the types of MSU RNAPs elongation subunits suggesting a possible role in $\mathrm{Zn}$ mediated proof-reading mechanism for chloroplast MSU RNAPs also, as proposed for DNA polymerases ${ }^{5}$. It is interesting to note that the 'gatekeeper pair' YG and the catalytic R are strictly conserved, including distance conservation in all the eubacterial and chloroplast $\beta^{\prime}$ and $\beta$ 'subunits of the MSU RNAPs. In addition to, an additional invariant LG pair is also observed suggesting its possible role in recognizing both the strands as reported for many viral polymerases. Another interesting feature is the immediate downstream amino acid from catalytic $\mathrm{R}$ in both the eubacterial and chloroplast and $\beta$ "subunits is a hydroxyamino acid, $\mathrm{S} / \mathrm{T}$, possibly playing a role in substrate binding. In both the cases, the catalytic $\mathrm{R}$ is placed at $16^{\text {th }}$ position downstream from the YG pair. This observation is in contrast to the earlier report for DNA polymerases and SSU RNAPs where 
it is positioned at $-7 / 8$ amino acids only 5 , 19. A long stretch of conservation is seen at the C-terminal ends of $\beta^{\prime}$ of eubacterial RNAPs (except in Blastomonas fulva, an aerobic photosynthetic bacteria) and many of the $\beta$ " subunits of chloroplast MSU RNAPs with few exceptions.

\section{Analysis of the Active Sites of MSU RNAPs of Eubacteria and Chloroplasts}

It is clear from the above MSA data, that the chloroplast MSU RNAPs are very much similar in functions to the eubacterial MSU RNAPs, such as the completely conserved substrate, template binding motifs, complete conservation of the catalytic amino acid $\mathrm{R}$ and in the distance conservations between the catalytic $\mathrm{R}$ and the YG pair in the initiation and elongation subunits. The metal binding region though found in different subunits is completely conserved. Furthermore, their susceptibility to various transcriptional inhibitors is also found to be similar. These points are further elaborated in the following section.

\subsection{Substrate, Template Binding and Catalytic Amino Acids are same in Eubacterial and Chloroplast MSU RNAPs}

As both the chloroplast and eubacterial MSU RNAPs carry out template-dependent nucleotide polymerization, it might be expected that both the enzymes would be structurally related to each other and hence, might be using similar types of amino acids for substrate, template binding and catalysis. Even though not much data are available on the 3D structures, biochemical, genetic and SDM analyses on the chloroplast MSU RNAPs, the major conclusions arrived in this communication are mainly based on the sequence similarities and completely conserved amino acid motifs in the critical regions between them and from the data available from their eubacterial counterparts. For example, the MSA analysis has shown that the eubacterial $\beta^{\prime}$ and chloroplast $\beta^{\prime}$ and $\beta$ " catalytic subunits harbour the mandatory amino acids, viz. the catalytic amino acid $\mathrm{R}$ and the DNA template binding YG pair, an R or S/T for NTP selection (highlighted in yellow) and the completely conserved metal binding sites. This is in close agreement with the earlier report by Palanivelu for the eubacterial MSU DNA dependent RNAPs ${ }^{-6}$. The interesting observation in chloroplast MSU RNAPs is that both the $\beta^{\prime}$ and $\beta$ " might be possibly participating in the elongation process by sharing the active sites which are accomplished by a single $\beta$ ' subunit in eubacteria. Thus, in the elongation subunit $\beta$ ", the distance between the YG and catalytic R, is doubled, i.e., it is placed at $+16^{\text {th }}$ position as found in the eubacterial elongation $\beta^{\prime}$ subunits ${ }^{-}$. The +1 amino acid in this case, is a hydroxyl amino acid $\mathrm{S} / \mathrm{T}$ which is placed adjacent to the $\mathrm{YG}$ pair which is reported to be involved in nucleotide discrimination in SSU RNAPs ${ }^{25}$. The $\beta$ subunits of both eubacteria and chloroplasts and $\beta^{\prime}$ and $\beta^{\prime \prime}$ subunits of eubacteria and chloroplasts, respectively show multiple YG pairs suggesting possibly multiple contacts with the DNA templates. An invariant $\mathrm{R}$ at $-7^{\text {th }}$ position from the catalytic $R$ is absent only in the $\beta$ ' subunits of eubacterial MSU polymerases but present in the $\beta$ " subunits of chloroplast enzymes (Table 1). The similar invariant $\mathrm{R}$, at -4 in SSU RNAPs and DNA polymerases (Table 1) is implicated in the NTP selection ${ }^{5,19}$.

\subsection{The Presence of Invariant Catalytic $\mathrm{R}$ and YG Pair and their Distance Conservations are same in both the Enzymes}

It has been found that almost all DNA polymerases and SSU RNAPs use a $\mathrm{K}$ for catalysis, i.e., in the initial proton transfer reactions ${ }^{5,19}$. However, in all MSU RNAPs there is no $\mathrm{K}$ but an $\mathrm{R}$ at the expected distance from the template binding YG pair (Table 1). However, a detailed analysis has shown that all prokaryotic DNA polymerases-II also use an invariant $\mathrm{R}$ in catalysis with similar distance conservations ${ }^{6}$; interestingly, an enzyme is associated $3^{\prime} \rightarrow 5^{\prime}$ exonuclease activity along with primase activity.

Table 1 shows the invariant YG pair and its catalytic $\mathrm{R}$ in $\beta, \beta$ ' and $\beta$ and $\beta$ " subunits of eubacterial and chloroplast MSU RNAPs, respectively (Figures 2-7).

It is interesting to note that the invariant YG pair appears to be specific for polymerases using DNA as the template and also for the prokaryotic MSU RNAPs, as it is not reported in RNA dependent RNAPs where they use RNA as the template ${ }^{26}$. In fact, the two YG pairs found in 
T7 polymerase are required for the enzyme activity and interestingly, exhibit no activity when single-stranded DNA was used as the substrate ${ }^{25}$. Three invariant Cs between catalytic R and YG pair in the elongation subunits in eubacterial $\beta^{\prime}$ is implicated in $\mathrm{Zn}$ binding ${ }^{20}$ and also found at the same distances in chloroplast $\beta$ " (Table 1), suggesting a similar function. Both the initiation and elongation subunits show an invariant $R$ at $-7 / 8$ from the catalytic $\mathrm{R}$ and adjacent (downstream) to YG pair in both the MSU RNAPs but absent in $\beta$ ' subunits of eubacteria (Table 1).

\subsection{Metal Binding Sites in MSU RNAPs of Eubacteria and Chloroplasts are same}

The metal binding site $\left(\mathrm{Mg}^{2+}\right.$ binding site) arrived at by the MSA analysis of eubacterial MSU RNAPs was also confirmed X-ray crystallographic analysis ${ }^{20}$ and also by SDM experiments ${ }^{27}$. Interestingly, the same $\mathrm{Mg}^{2+}$ binding site that is found in both the initiation subunits, viz. in the $\beta$ subunits of eubacteria and chloroplasts, suggests a similar role in both. Furthermore, similarities are also found in the metal binding sites in the elongation subunits of both the MSU RNAPs, i.e., a $\mathrm{Mg}^{2+}$ and a $\mathrm{Zn}^{2+}$ binding motifs are very similar in both the elongation subunits of eubacteria and chloroplasts (Table 2). Crystallographic analysis of the T. aquaticus RNAP have shown that the Mg atom is chelated at an absolutely conserved - NADFDGD - motif in the $\beta$ ' subunits ${ }^{20}$. Interestingly, the same sequence is absolutely conserved in the $\beta$ ' subunits of chloroplast RNAPs, further confirming an identical function in both the RNAPs. The Zn binding motif that is identified by $\mathrm{X}$-ray crystallographic method in the eubacterial $\beta$ ' is also identified but in the chloroplast $\beta$ " subunits. Interestingly, the three invariant Cs are arranged at the same distances in both eubacterial $\beta$ ' and in the chloroplast $\beta$ " subunits, suggesting a similar function. In E. coli $\beta$ ' subunit, the amino acids 814,888 and 895 are shown to be involved in $\mathrm{Zn}$ binding (Table 2). The Zn binding motif that is found in elongation subunits $\beta$ ' and $\beta$ " may play a role in the $\mathrm{Zn}$ mediated proof-reading in these RNAPs as suggested for DNA polymerases ${ }^{5}$.

\subsection{Both the Eubacterial and Chloroplast MSU RNAPs are Similar in their Susceptibility to various Transcriptional Inhibitors}

Bothchloroplastand eubacterialMSURNAPsareinhibited by the same types of transcriptional inhibitors, further
Table 1. Amino acids around the catalytic amino acid $\mathrm{K} / \mathrm{R}$ and the YG pair in DNA polymerases and in SSU and MSU RNAPs

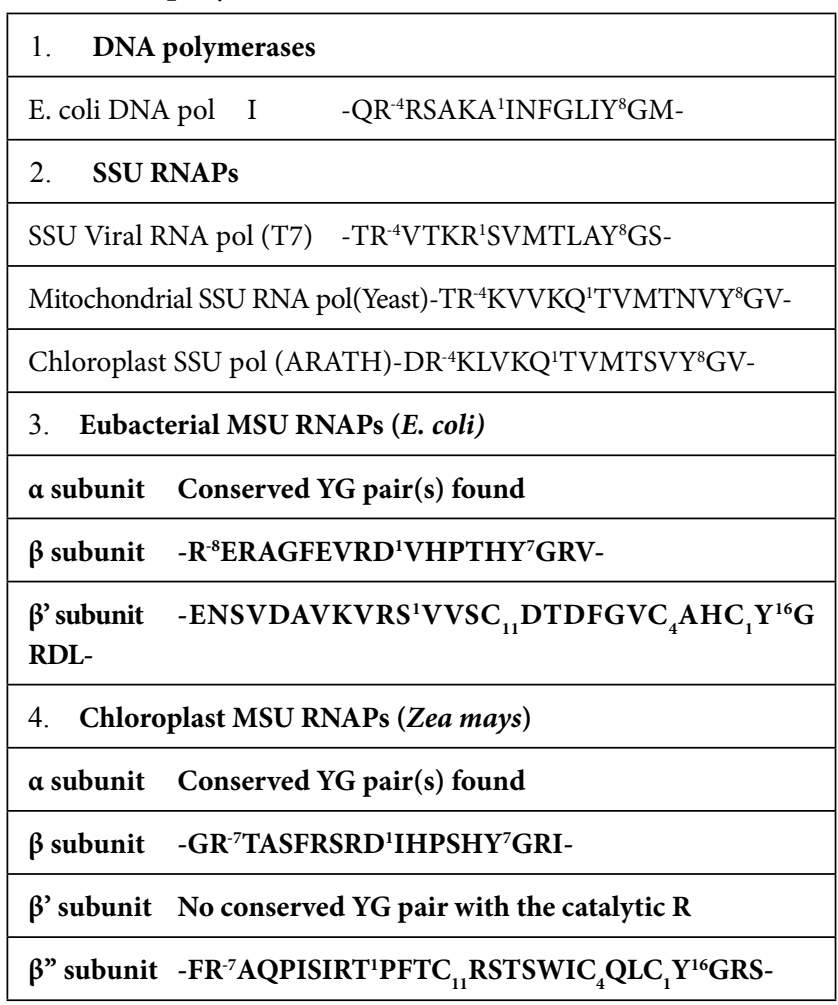

NB: The MSU RNAPs which use R in the catalytic site is shown in bold.

The invariant $R$, at $-6 / 7$ is not found in $\beta$ ' subunits of eubacteria but found at -7 in the chloroplast MSU RNAPs

suggesting structural and functional similarities between them. In fact, in vitro and in vivo studies with rifampicin, a well-known inhibitor of bacterial MSU RNAPs, have also been shown to inhibit the chloroplast RNAPs 28,29 (but interestingly, did not inhibit the NEP). Further support is provided by the sensitivity of the chloroplast RNAPs to tagetitoxin, a phytotoxin isolated from the plant pathogenic bacterium, Pseudomonas syringae pv. Tagetis also inhibits eubacterial transcription ${ }^{30}-\underline{32}$. (Unlike rifampicin which inhibits the initiation step in transcription, the tagetitoxin inhibits both the initiation and elongation stages in both the types of MSU enzymes, as its target is the NTP loading site). These results confirm a high degree of conservation between the plastidencoded and eubacterial MSU RNAPs. Moreover, the bacterial 'stringent control' mechanism which enables them to adapt to nutrient-limiting stress conditions, use the effector molecule, guanosine-5'-diphosphate-3' 
Table 2. Similarities in the metal binding sites of MSU RNAPs from eubacteria and chloroplasts

\begin{tabular}{|c|c|c|}
\hline Subunit/Organism & Metal biding site & Method and Reference \\
\hline$\beta$ eubacteria (E. coli) & - LEHDDAN- \& -GYNFEDS ${ }^{\star}\left(\mathrm{Mg}^{2+}\right)$ & MSA (This communication) \\
\hline$\beta^{\prime}$ eubacteria (E. coli) & $\begin{array}{l}\text { YNADFDGDQM- }\left(\mathbf{M g}^{2+}\right) \text { \&X-ray } \\
\text { crystallographic data }\end{array}$ & $\begin{array}{l}- \text { RS }^{1} \text { PLTC } \\
\text { AHDFGVC } \\
\text { AHC }^{15} \mathbf{Y}^{16} \mathbf{G}-\left(\mathbf{Z n}^{2+}\right)\end{array}$ \\
\hline$\beta$ chloroplasts (Zeamays) & -IEHNDAN- \&-GYNFEDA*- $\left(\mathrm{Mg}^{2+}\right)$ & MSA (This communication) \\
\hline$\beta^{\prime}$ chloroplasts (Zeamays) & -FNADFDGDQM* - $\left(\mathrm{Mg}^{2+}\right)$ & MSA (This communication) \\
\hline$\beta "$ chloroplasts (Zea mays) & - RS $^{1}$ PLTC $^{5}$ RSTSWIC $^{12} \mathbf{Q L C}^{15} \mathbf{Y}^{16} \mathbf{G}-\left(\mathbf{Z n}^{2+}\right)$ & MSA (This communication) \\
\hline
\end{tabular}

*The possible metal binding site is arrived at by MSA and SDM*

NB: The $\beta$ ' subunits of eubacteria contain both the $\mathrm{Mg}^{2+}$ and $\mathrm{Zn}^{2+}$ binding sites on the same subunit whereas in chloroplasts $\mathrm{Mg}^{2+}$

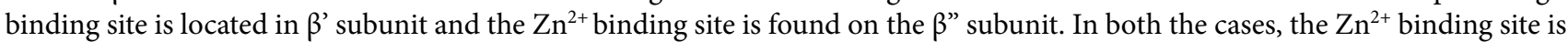
built within the catalytic region with the precise distance conservations suggesting a possible role in the Zn-mediated proof-reading mechanism during elongation the process.

-diphosphate (ppGpp), also showed a similar function in chloroplasts. Interestingly, the ppGpp inhibits the transcriptional activity of PEP through direct binding onto the $\beta$ 'subunit ${ }^{33}$. Thus, under stress conditions, PEPs are also under the control of bacterial-like stringent response, mediated by ppGpp. The above evidences further support that the two MSU RNAPs are very similar in structure and function.

\subsection{Promoter Sequences and Promoter Recognition Events by both the Enzymes are Similar}

The eubacterial and plastid promoters are found to be very similar in their structure and functions. For example, both the MSU RNAPs recognize $\sigma 70$ type promoters, i.e., their promoters have the same type of -10 and -35 consensus sequence elements. Moreover, both the promoters contain similar sequences at -35 (TTGACA) and -10 (TATAAT), which are recognized by $\sigma$ subunit (for PEPs, the $\sigma$ subunit is nuclear encoded). Furthermore, the E. coli RNAP is able to faithfully recognize the PEP promoters ${ }^{34}$ and thus further corroborating similarity in their structure and function in promoter recognition and transcription initiation events.

\subsection{The $\beta, \beta$ ' and $\beta$ " in PEPs Possibly Work in Tandem as in Eubacteria}

As proposed for the MSU RNAPs of eubacteria, the $\beta, \beta$ ' and $\beta$ " in PEPs also possibly work in tandem as shown in Figure 8. The only difference is the $\beta$ ' subunit in chloroplast MSU RNAPs is actually needed for the $\mathrm{Mg}^{2+}$ binding at the NTP loading site during elongation. Figure 8 shows a consensus model of the PEP subunits from promoter recognition to initiation and elongation events during the transcription process in chloroplasts.

While the $\sigma$ subunit recognizes and binds to the promoter, the $\beta$ subunit binds to the TSS and initiates transcription. It has been found in eubacterial MSU RNAPs that the initiation by the $\beta$ subunits is not smooth and it makes many aborted transcripts of sizes 2-7 nucleotides (nts) before the elongation step takes over 22 , 29. It has been shown that the eubacterial MSU RNAPs depend on this short RNA/DNA hybrids for stability and further processivity, as the Ternary Elongation Complex (TEC) with RNA/DNA hybrids of less than 8-bp display markedly less stability than those that are $8 \mathrm{bp}$ or longer $\frac{35}{3}$. The experimental evidence corroborates the MSA findings that the distance between the catalytic $R$ and the YG pair is 7 amino acids and is strictly conserved in all $\beta$ subunits of eubacteria as well as in chloroplasts. This consensus model is further confirmed in E. coli MSU RNAP by quantitation of the preinitiation complex by high-resolution gel electrophoresis technique which showed that many oligonucleotides are formed per preinitiation complex, up to hexanucleotides and only longer transcripts escape the cycling reaction and go for elongation ${ }^{23}$. MSA analysis shows that the heptanucleotides fit into the elongation subunit active site and further elongated and possibly not detected in high-resolution gel electrophoresis technique. 


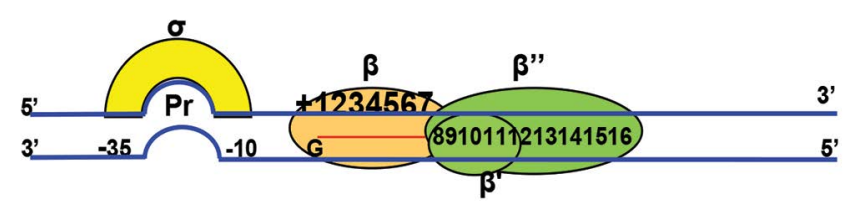

Figure 8. A consensus model showing promoter recognition, initiation and elongation events by $\sigma, \beta, \beta$, and $\beta^{\prime \prime}$ subunits of MSU RNAPs from chloroplasts.

NB: Thick lines indicate DNA strands and the thin line indicates the RNA transcript.

$\mathrm{G}$ is shown as the first nucleotide at the +1 TSS. $\mathrm{Pr}$, The promoter region

\section{Mechanism of Nucleotide Polymerization in the MSU RNAPs of Chloroplasts}

MSA of both the MSU RNAPs have shown that they possess the same amino acid motifs, catalytic amino acid and metal binding regions and hence it is highly likely both use the same catalytic mechanism for nucleotidyl transfers as exemplified by Palalnivelu ${ }^{6}$. Thus, as shown for the eubacterial MSU RNAPs, a minimal number of steps in the catalytic cycle of chloroplast RNAPs also consists of NTP selection, Watson-Crick base pairing with the complementary nucleotides with the template DNA, catalysis, pyrophosphate release and translocation $-\frac{636}{,}$ and references therein. Polymerization reactions in PEPs should also be very similar in both the initiation and elongation subunits as the use same amino acids, catalytic and metal binding motifs.

\section{Conclusions}

MSA have shown that in both the MSU RNAPs of eubacteria and chloroplasts, the active sites, catalytic amino acids and metal binding regions are absolutely conserved both in the initiation and elongation subunits. Furthermore, same distance conservations are also observed among the initiation and elongation subunits of both the enzymes. Large numbers of highly conserved monos, diads, triads may play an important role in folding the proteins to the correct 3D structure. Therefore, it is suggested that the MSU RNAPs of chloroplasts may also follow very similar polymerization and proof-reading mechanisms as proposed for eubacteria. MSA data and the available experimental data show that both the eubacterial and chloroplast MSU RNAPs would have possibly evolved from a common ancestor.

\section{Acknowledgements}

The author wishes to thank Dr. H. Shakila, Professor and Head, Department of Molecular Microbiology, School of Biotechnology, Madurai Kamaraj University, Madurai for useful suggestions on the manuscript.

\section{References}

1. Werner F, Grohmann D. Evolution of multisubunit RNA polymerases in the three domains of life. Nature Reviews. 2001; 9:85-98. PMid: 21233849. https://doi.org/10.1038/ nrmicro2507

2. Borner T, Aleynikova AY, Zubo YO, Kusnetsov VV. Chloroplast RNA polymerases: Role in chloroplast biogenesis. Biochimica et Biophysica Acta. 2015; 1847:761-9. PMid: 25680513. https://doi.org/10.1016/j. bbabio.2015.02.004

3. Liere K, Weihe A, Borner T. The transcription machineries of plant mitochondria and chloroplasts: Composition, function and regulation. Journal of Plant Physiology. 2011; 168:1345-60. PMid: 21316793. https://doi.org/10.1016/j. jplph.2011.01.005

4. Daniell D, Choun-Sea Lin, Ming Yu, Wan-Jung Chang. Chloroplast genomes: Diversity, evolution and applications in genetic engineering. Genome Biology. 2016; 17:134. PMid: 27339192 PMCid: PMC4918201. https://doi. org/10.1186/s13059-016-1004-2

5. Palanivelu P. Single subunit RNA Polymerases - An insight into their active sites and mechanism of action. Biotechnology Journal International. 2017; 20:1-35. https:// doi.org/10.9734/BJI/2017/38632

6. Palanivelu P. Multi-subunit RNA Polymerases of Bacteria An insight into their active sites and catalytic mechanism. Indian Journal of Science and Technology. 2018; 11 (39):137. https://doi.org/10.9734/BJI/2017/38632

7. Ozeki H, Ohyama K, Inokuchi H, Fukuzawa H, Kohchi T, Sano T, Nakahigashi K, Umesono K. Genetic system of chloroplasts. Cold Spring Harbor Symposium for Quantitative Biology. 1987; 52:791-804. PMid: 3502722. https://doi.org/10.1101/SQB.1987.052.01.088

8. Hedtke B, Borner T, Weihe A. Mitochondrial and chloroplast phage-type RNA polymerase in Arabidopsis. Science. 1997; 277:809-11. PMid: 9242608. https://doi. org/10.1126/science.277.5327.809

9. Hajdukiewicz PT, Allison LA, Maliga P. The two RNA polymerases encoded by the nuclear and the plastid 
compartments transcribe distinct groups of genes in tobacco plastids. The EMBO Journal. 1997; 16:4041-8. PMid: 9233813 PMCid: PMC1170027. https://doi. org/10.1093/emboj/16.13.4041

10. Allison LA, Simon LD, Maliga P. Deletion of rpoB reveals a second distinct transcription system in plastids of higher plants. The EMBO Journal. 1996; 15:28029. PMid: 8654377 PMCid: PMC450217 https://doi. org/10.1002/j.1460-2075.1996.tb00640.x

11. De Santis-Maciossek G, Kofer W, Bock A, Schoch S, Maier RM, Wanner G, Rudiger W, Koop HU, Herrmann RG. Targeted disruption of the plastid RNA polymerase genes rpoA, B and C1: Molecular biology, biochemistry and ultrastructure. The Plant Journal. 1999; 18:477-89. PMid: 10417698. https://doi.org/10.1046/ j.1365-313X.1999.00473.x

12. Severinov K, Mustaev A, Kukarini A, Muzzin O, Little MC, Hallick RB. Chloroplast rpoA, rpoB, and rpoC genes specify at least three components of a chloroplast DNA-dependent RNA polymerase active in tRNA and mRNA transcription. The Journal of Biological Chemistry. 1998; 263:14302-7.

13. Legen JS, Kemp K, Krause B, Profanter RG, Maier HRM. Comparative analysis of plastid transcription profiles of entire plastid chromosomes from tobacco attributed to wild-type and PEP-deficient transcription machineries. The Plant Journal. 2002; 31:171-88. PMid: 12121447. https://doi.org/10.1046/j.1365-313X.2002.01349.x

14. Serino G, Maliga P. RNA polymerase subunits encoded by the plastid rpo genes are not shared with the nucleusencoded plastid enzyme. Plant Physiology.1998; 117:1165-70. PMid: 9701572 PMCid: PMC34880. https:// doi.org/10.1104/pp.117.4.1165

15. Allison LA. The role of sigma factors in plastid transcription. Biochimie. 2000; 82:537-48 https://doi.org/10.1016/S03009084(00)00611-8

16. Gruissem W, Zurawski G. Analysis of promoter regions for the spinach chloroplast rbcL, atpB and psbA genes. The EMBO Journal. 1985; 4:3375-83. PMid: 2868888 PMCid: PMC554673. https://doi.org/10.1002/j.1460-2075.1985. tb04093.x

17. Gruissem W, Zurawski G. Identification and mutational analysis of the promoter for a spinach chloroplast transfer RNA gene. The EMBO Journal. 1985; 4:163744. PMid: 2992936 PMCid: PMC554398. https://doi. org/10.1002/j.1460-2075.1985.tb03831.x

18. Cramer P. Multisubunit RNA polymerases. Current Opinion in Structural Biology. 2002; 12:89-97. https://doi. org/10.1016/S0959-440X(02)00294-4

19. Palanivelu P. DNA polymerases - An insight into their active sites and mechanism of action. International Journal of Biochemistry Research and Reviews. 2013; 3:205-47. https://doi.org/10.9734/IJBCRR/2013/3777

20. Zhang G, Campbell EA, Minakhin L, Richter C, Severinov K, Darst SA. Crystal structure of Thermus aquaticus core RNA polymerase at 3.3 A resolution. Cell. 1999; 98:811-24. https://doi.org/10.1016/S0092-8674(00)81515-9

21. Záhonová K, Fussy Z, Oborník M, EliasM, Yurchenko $\mathrm{V}$. RuBisCO in non-photosynthetic alga euglena longa: divergent features, transcriptomic analysis and regulation of complex formation. PLoS One. 2016; 11:e0158790. PMid: 27391690 PMCid: PMC4938576. https://doi.org/10.1371/ journal.pone.0158790

22. Tintut Y, Wang JT, Gralla JD. Abortive cycling and the release of polymerase for elongation at the $\sigma$ 54-dependent glnAp2 promoter. The Journal of Biological Chemistry. 1995; 270:24392-8. PMid: 7592652. https://doi.org/10.1074/ jbc.270.41.24392

23. Carpousis AJ, Gralla JD. Interaction of RNA polymerase with lacUV5 promoter DNA during mRNA initiation and elongation. Foot-printing, methylation and rifampicinsensitivity changes accompanying transcription initiation. The Journal of Molecular Biology. 1985; 183:165-77. https://doi.org/10.1016/0022-2836(85)90210-4

24. Finn RD, Orlova EV, Gowen B, Buck M, van Heel M. Escherichia coli RNA polymerase, core and holoenzyme structures.The EMBO Journal. 2000; 19:6833-44. PMid: 11118218 PMCid: PMC305883. https://doi.org/10.1093/ emboj/19.24.6833

25. Kostyuk SM, Dragan DL, Lyakhov, VO, Rechinsky VL, Tunitskaya BK. Chernov SN, Kochetkov E. Mutants of T7 RNA polymerase that are able to synthesize both RNA and DNA. FEBS Letters. 1995; 369:165-8. https://doi. org/10.1016/0014-5793(95)00732-O

26. Tunitskaya VL, Kochetkov SN. Structural and functional analysis of bacteriophage T7 RNA polymerase. Biochemistry (Moscow). 2002; 67:1124-35. https://doi. org/10.1023/A:1020911223250

27. Svetlov V, Vassylyev DG, Artsimovitch I. Discrimination against deoxyribonucleotide substrates by bacterial RNA polymerase. The Journal of Biological Chemistry. 2004; 279:38087-90. PMid: 15262972 PMCid: PMC3131404. https://doi.org/10.1074/jbc.C400316200

28. Surzycki SJ. The chloroplast of Chlamydomonas reinhardi: Effect of rifampicin on chloroplast DNA-dependent RNA polymerases. Proceedings of the National Academy of Sciences (USA). 1969; 63:1327-34. PMid: 5260935 PMCid: PMC223468. https://doi.org/10.1073/pnas.63.4.1327

29. Campbell EA, Korzheva N, Mustaev A, Murakami K, Nair S, Goldfarb A, Darst SA. Structural mechanism for rifampicin inhibition of bacterial RNA polymerase. 
Cell. 2001; 104:901-12. https://doi.org/10.1016/S00928674(01)00286-0

30. Mathews DE, Durbin RD. Tagetitoxin inhibits RNA synthesis directed by RNA polymerase from chloroplasts and Escherichia coli. The Journal of Biological Chemistry. 1990; 265:493-8.

31. Domecq C, Trinh V, Langelier MF, Archambault J, CoulombeB. Inhibitors of multisubunitrna polymerases as tools to study transcriptional mechanisms in prokaryotes and eukaryotes. Current Chemical Biology. 2008; 2:2031. https://doi.org/10.2174/187231308783334144

32. Ma C, Yang X, Lewis, PJ. Bacterial transcription as a target for antibacterial drug development. Microbiology and Molecular Biology Reviews. 2016; 80:139-60. PMid: 26764017 PMCid: PMC4771368. https://doi.org/10.1128/ MMBR.00055-15

33. Imamura S, Asayama M. Sigma factors for cyanobacterial transcription. Gene Regulations and System Biology. 2009; 3:65-87. https://doi.org/10.4137/GRSB.S2090
34. Bradley D, Gatenby AA. Mutational analysis of the maize chloroplast ATPase beta subunit gene promoter: the isolation of promoter mutants in E. coli and their characterization in a chloroplast in vitro transcription system. The EMBO Journal. 1985; 4:36418. PMid: 3004965 PMCid: PMC554712. https://doi. org/10.1002/j.1460-2075.1985.tb04129.x

35. Kireeva ML, Komissarova N, Waugh DS, Kashlev M. The 8-nucleotide-long RNA:DNA hybrid is a primary stability determinant of the RNA polymerase II elongation complex. The Journal of Biological Chemistry. 2000; 275:6530-6. PMid: 10692458.https://doi.org/10.1074/jbc.275.9.6530

36. Sosunov V, Sosunova E, Mustaev A, Bass I., Nikiforov V, Goldfarb, A. Unified two-metal mechanism of RNA synthesis and degradation by RNA polymerase. The EMBO Journal. 2003; 22:2234-44. PMid: 12727889 PMCid: PMC156065. https://doi.org/10.1093/emboj/ cdg193 